\title{
NEURAL NETWORK MUSIC GENRE CLASSIFICATION
}

\author{
A Thesis \\ Submitted to the Faculty of Graduate Studies and Research \\ In Partial Fulfillment of the Requirements \\ for the Degree of \\ Master of Applied Science \\ in Software Systems Engineering \\ University of Regina \\ by \\ Nikki Pelchat \\ Regina, Saskatchewan \\ January 2021
}

Copyright (C) 2021: Nikki Pelchat 


\section{UNIVERSITY OF REGINA}

\section{FACULTY OF GRADUATE STUDIES AND RESEARCH SUPERVISORY AND EXAMINING COMMITTEE}

Nikki Pelchat, candidate for the degree of Master of Applied Science in Software Systems Engineering, has presented a thesis titled, Neural Network Music Genre Classification, in an oral examination held on December 8, 2020. The following committee members have found the thesis acceptable in form and content, and that the candidate demonstrated satisfactory knowledge of the subject material.

External Examiner:

Supervisor:

Committee Member:

Chair of Defense:

*via ZOOM Conferencing
${ }^{*}$ Dr. Paul Laforge, Electronic Systems Engineering

*Dr. Craig Gelowitz, Software Systems Engineering

${ }^{*}$ Dr. Timothy Macaig, Software Systems Engineering

${ }^{*}$ Dr. Rebecca Caines, Creative Technologies 


\begin{abstract}
Music recommendation systems have become popular in recent years with the increasing variety of music content being produced as well as the sheer size of digital music collections which are available at the touch of a finger. Large collections of digital music are commonly organized using genre labels. In addition, music genres are regularly used by recommendation systems to suggest new music to the listeners. The chore of classifying a large amount of music manually can be difficult and time consuming. It is for these reasons, the automatic classification of music by genre is a crucial task. The ability to automatically classify music by genre using machine learning can be quicker and arguably more accurate than doing it manually.

Using neural networks for generic classification tasks is a well researched area within machine learning. In recent years, the classification of music by genre has become part of the same problem domain. Differences in song libraries, machine learning techniques, input formats, and types of neural networks implemented have all had varying levels of success.

This thesis implements a convolutional neural network that classifies music by genre through the examination of spectrogram images. It concentrates on three specific types of spectrogram inputs (Linear, Logarithmic, and Mel scaled spectrograms) as well as several input variables and neural network learning techniques to determine the effect that they have on the overall accuracy of the genre classification network. This thesis demonstrates these convolutional neural network techniques for music genre classification and assesses their viability and accuracy.
\end{abstract}




\section{ACKNOWLEDGEMENTS}

I would like to express sincere thanks to my supervisor, Dr. Craig M. Gelowitz, for making this research possible. His invaluable guidance, support, and dedicated time throughout every phase of my studies and research have been appreciated.

I would like to thank the University of Regina for the financial support I received during my research in the form of research awards.

I would like to thank Adam Tilson for always lending advice while tackling his own path of research. It was helpful to have a peer whose research also lay within the topic of deep learning.

I would like to thank my parents for their wise counsel and sympathetic ear, my husband for supporting me emotionally through my studies, and my sister for pushing me towards pursuing graduate studies. 


\section{DEDICATION}

To,

\section{Evan Pelchat}

whose patience, love, and support have made this research possible. 


\section{TABLE OF CONTENTS}

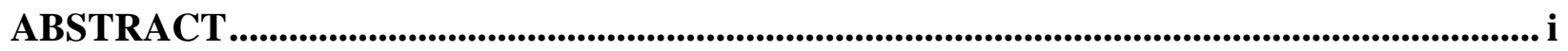

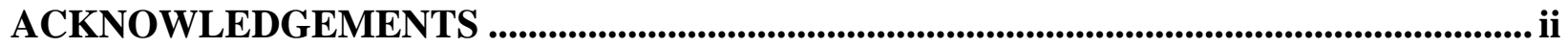

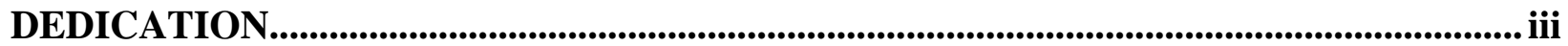

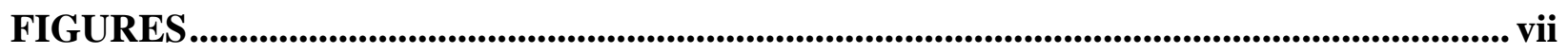

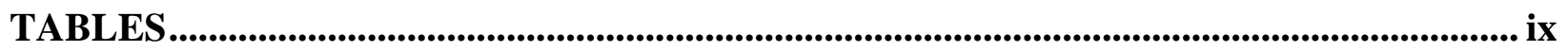

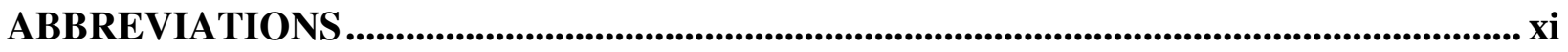

1 INTRODUCTION

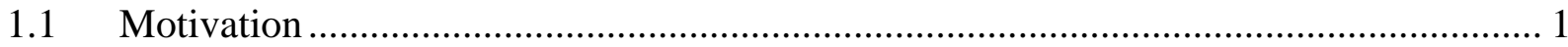

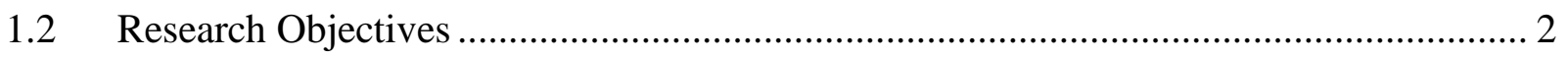

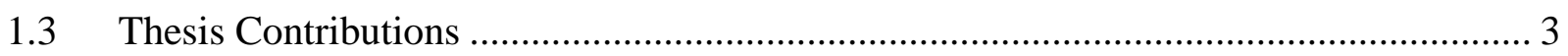

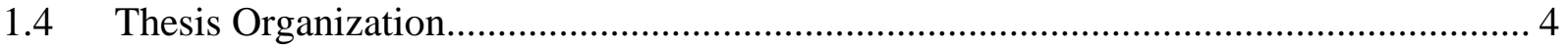

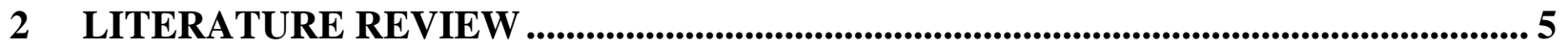

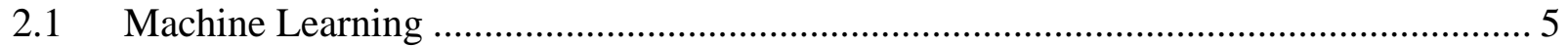

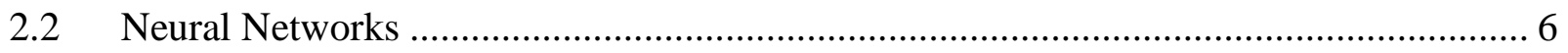

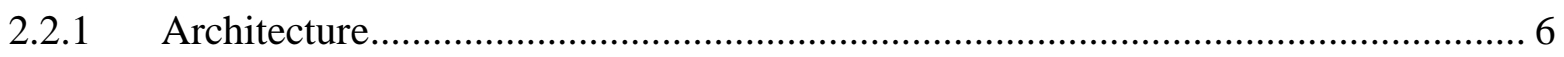

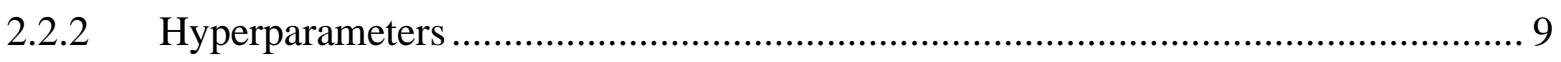

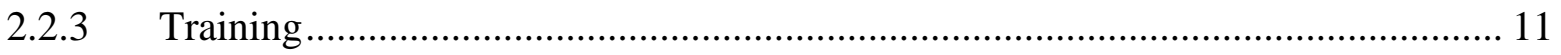

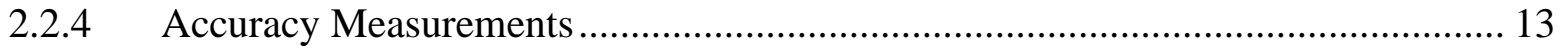

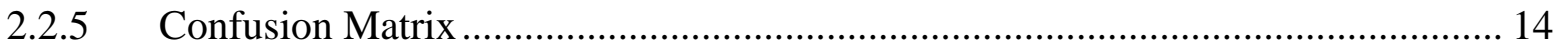

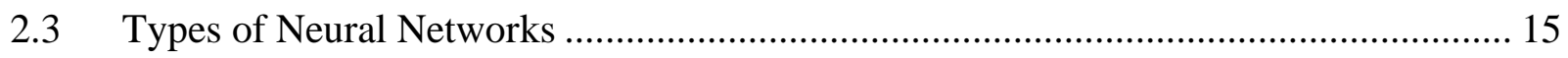

2.3.1 Convolutional Neural Network ........................................................................... 15

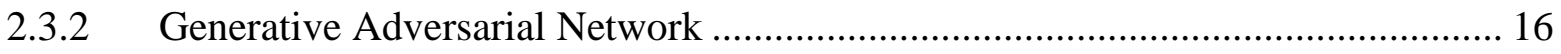

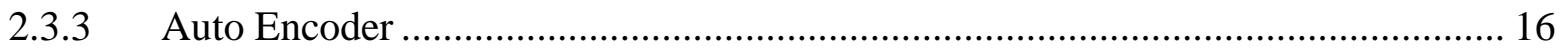

2.3.4 Recurrent Neural Network ....................................................................... 17

2.4 Formatting Music as Input into a Convolutional Neural Network............................ 18

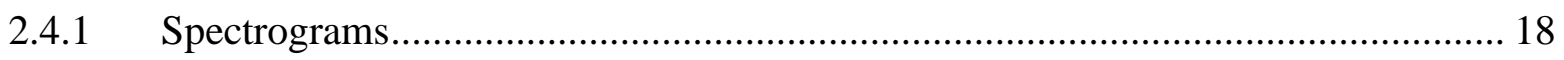

2.4.2 Spectrogram Frequency Scale Visualizations.................................................... 20

2.4.3 Mel Frequency Cepstral Coefficient (MFCC) ................................................... 21

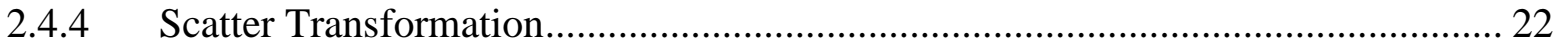

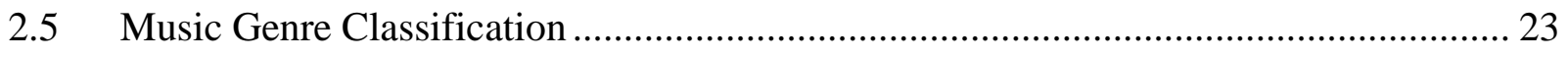


2.5.1 Music Genre Classification Focused on Engineered Features............................ 24

2.5.2 Music Classification using MFCC or Scatter Transformation ............................. 26

2.5.3 Music Classification using Spectrograms .......................................................... 31

3 METHODOLOGY \& IMPLEMENTATION WORK ...................................................... 42

3.1 Collecting Music Libraries .............................................................................. 43

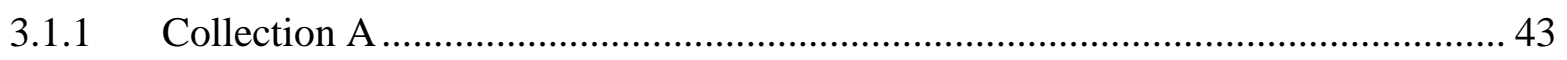

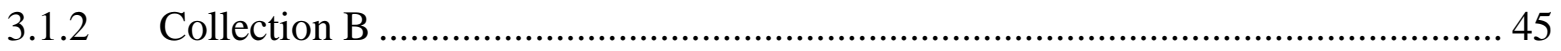

3.1.3 Differences in Collection A and B ............................................................. 46

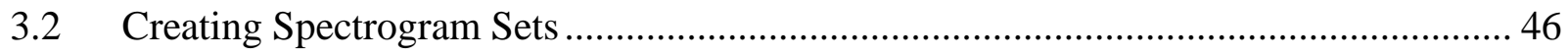

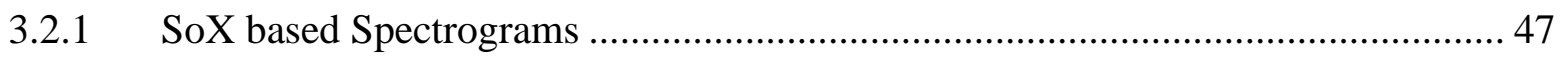

3.2.2 Librosa based Spectrograms .................................................................... 48

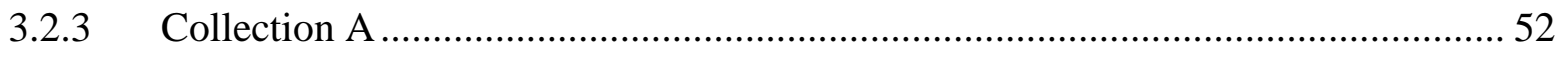

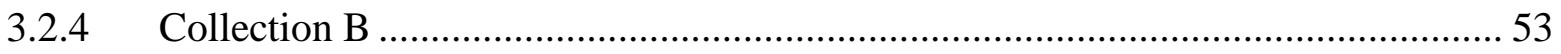

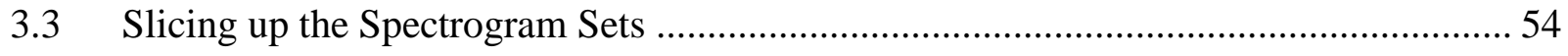

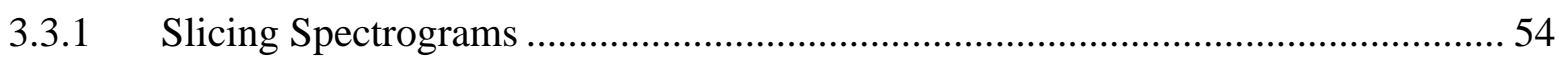

3.3.2 Contrast Filtering on Sliced Spectrograms ............................................... 56

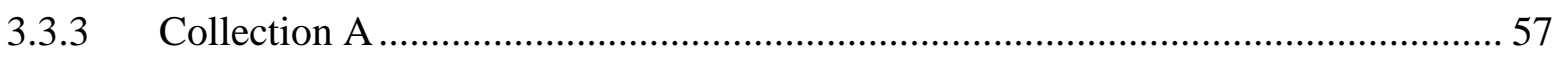

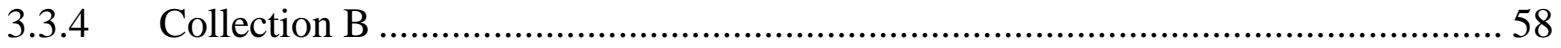

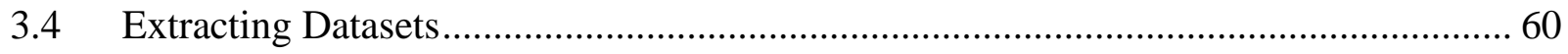

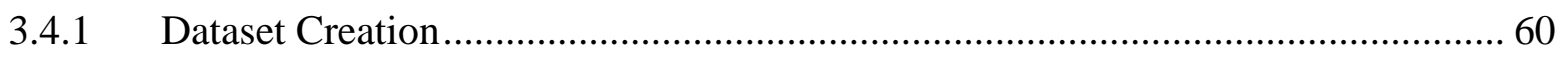

3.4.2 Extracting Datasets to Compare Collection A and Collection B ......................... 61

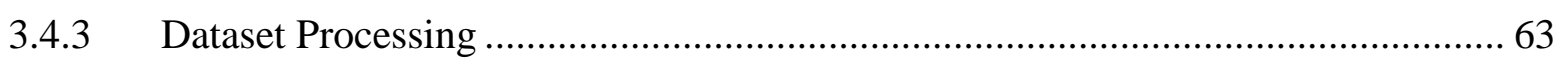

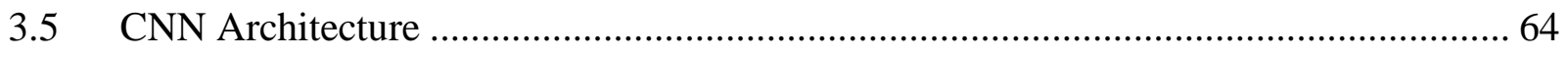

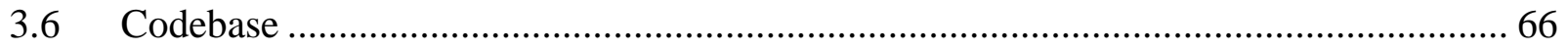

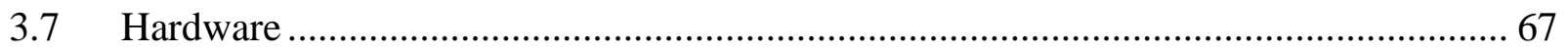

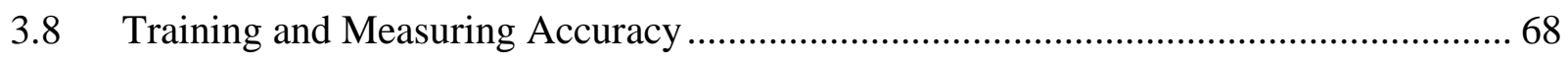

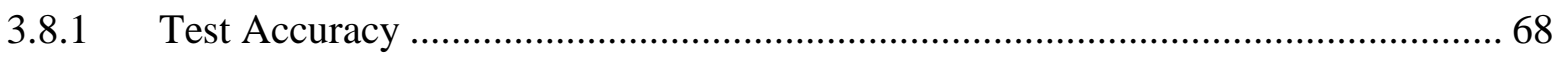

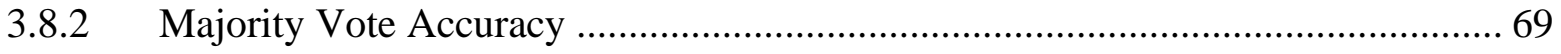

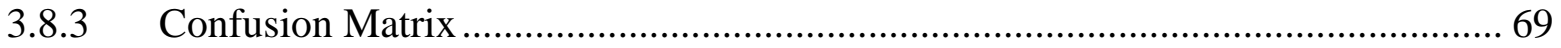

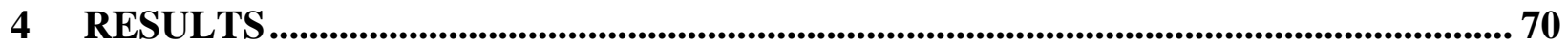

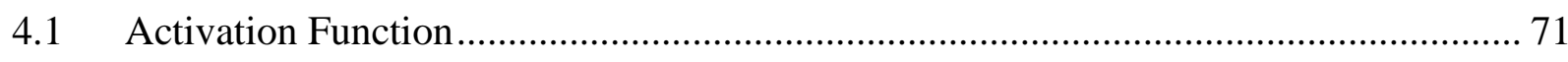

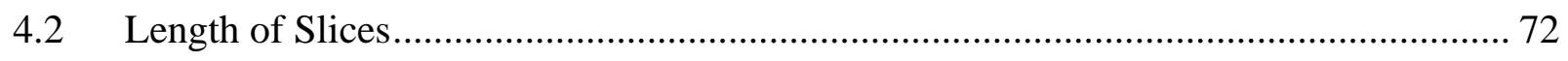




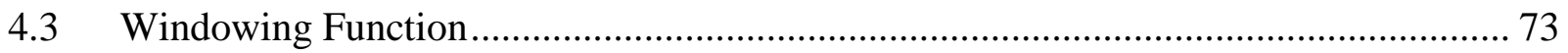

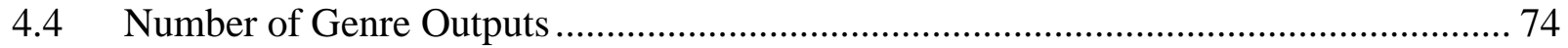

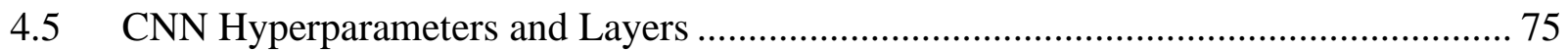

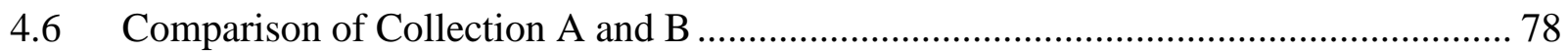

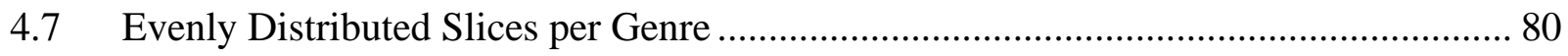

4.8 Linear, Mel, and Logarithmic Spectrogram Input....................................................... 82

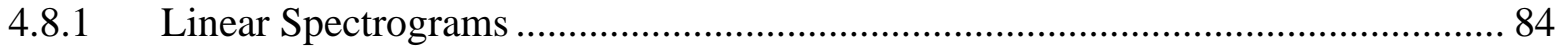

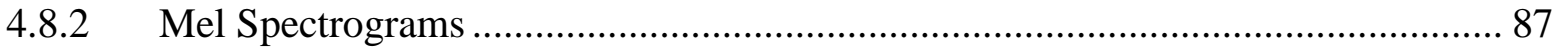

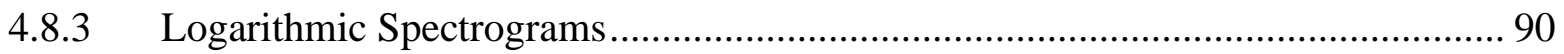

4.8.4 Comparison of Linear, Mel, and Logarithmic Spectrograms ................................ 94

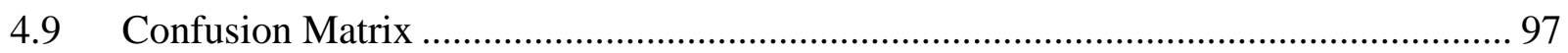

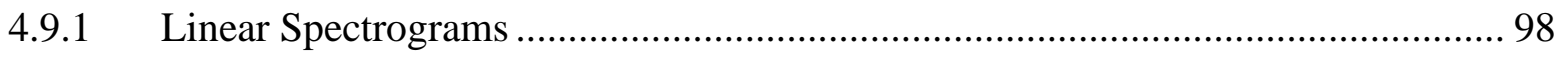

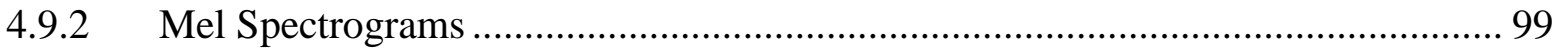

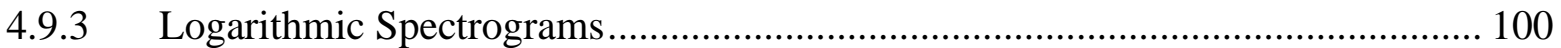

4.9.4 Comparison of Confusion Matrices ……………................................................. 101

5 CONCLUSIONS AND FUTURE WORK................................................................ 103

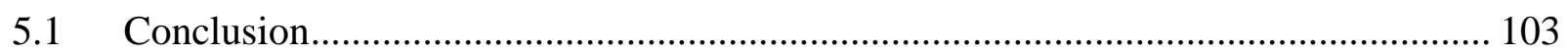

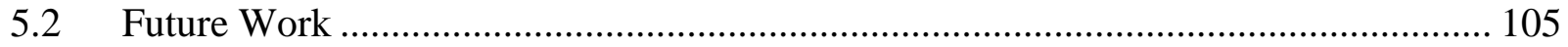

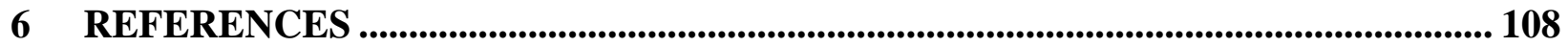




\section{FIGURES}

Figure 1 Equation used within a neural network node. ................................................. 6

Figure 2 A neural network node diagram. ....................................................................... 7

Figure 3 A simple neural network architecture.............................................................. 8

Figure 4 Different activation function graphs used by neural networks.................................. 9

Figure 5 Example of a Cost Function (C) of two variables (v1, v2). Source [17].................... 13

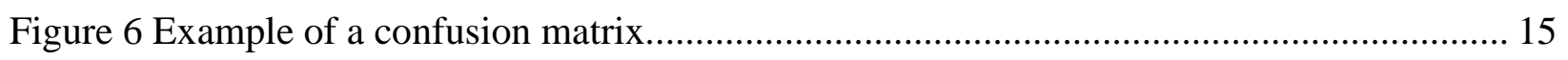

Figure 7 Example spectrogram plotted on a linear $\mathrm{Y}$ axis of $\mathrm{Hz}$ and a $\mathrm{X}$ axis in seconds. ......... 18

Figure 8 Example of a single signal, left; The signal being infinitely repeated over time and displaying a discontinuity, right. Source [30] ................................................................... 19

Figure 9 Window function, left; Window function repeated, right. This shows how a windowing function can help minimize the discontinuity. Source [30] ................................................ 20

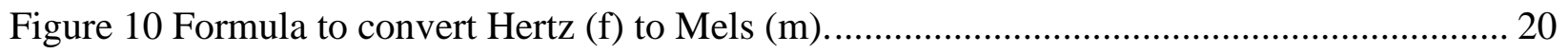

Figure 11 Using the same audio signal, the difference in frequency scale used to create a

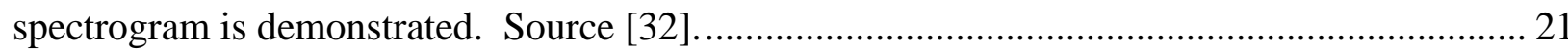

Figure 12 MFCCs extracted from an audio signal. Source [33] ........................................... 22

Figure 13 Accuracy before the majority vote from Dai and Liu. Source [18]......................... 29

Figure 14 An overview of the classification options investigated in [20]. Source [20]............. 32

Figure 15 Data augmentation techniques done on a spectrogram slice by [21]. Source [21]..... 35

Figure $16 \mathrm{CNN}$ architecture used by Despois. Source [42] ............................................... 37

Figure 17 Spectrogram of 12.8 seconds of a Pop song created by SoX with labelled axes. ........ 47

Figure 18 Example of a full 9,000 x 128 pixel spectrogram created by SoX........................... 48

Figure 19 Linear Frequency Spectrogram snippets comparing SoX versus Librosa libraries. .... 50

Figure 20 The same section of song converted into a spectrogram by SoX and Librosa for comparison.

Figure 21 (Top) 9,000 x 128 spectrogram; (Middle) Zoomed in on the first 12.8 seconds of the spectrogram; (Bottom) The 12.8 seconds being sliced into 2.56 second snippets. Figure 22 Enlarged portion of Figure 21; (Top) 12.8 second spectrogram of Pop music; (Bottom) divided into 2.56 second segments. 
Figure 23 Greyscale contrast between full black and full white labelled with their respective RBG value 56

Figure 24 (Top) A song that has a silent pause in the middle; (Bottom) A song finishes before the audio file is turned off. 57

Figure 25 Spectrogram slices which were filtered out because of the lack of contrast. ..............57

Figure 26 Preprocessing stages which took MP3 files into images in a dataset........................ 63

Figure 27 A diagram of the implemented CNN architecture................................................ 64

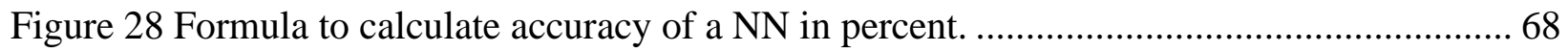

Figure 29 Formula to calculate majority vote accuracy of a $\mathrm{NN}$ in percent............................. 69

Figure 30 A depiction of how the number of neurons and hidden layers in a network can affect

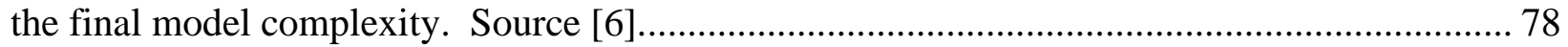

Figure 31 A linear spectrogram slice created by SoX and the same one created by Librosa...... 83

Figure 32 Average training and average validation accuracy per epoch for linear Librosa

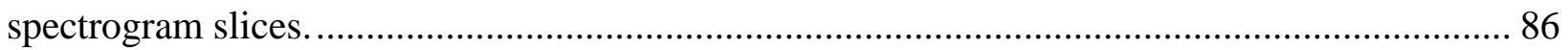

Figure 33 Validation loss per epoch for linear Librosa spectrogram slices............................. 87

Figure 34 Validation accuracy per epoch for Mel scaled Librosa spectrogram slices. ............... 89

Figure 35 Validation loss per epoch for Mel scaled Librosa spectrogram slices. ...................... 90

Figure 36 Validation accuracy per epoch for logarithmic Librosa spectrogram slices. .............. 93

Figure 37 Validation loss per epoch for logarithmic Librosa spectrogram slices. .................... 94

Figure 38 Comparison of validation accuracy per epoch for linear, Mel, and logarithmic Librosa

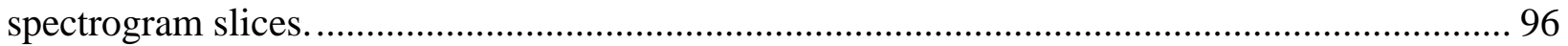

Figure 39 Comparison of validation loss per epoch for linear, Mel, and logarithmic Librosa

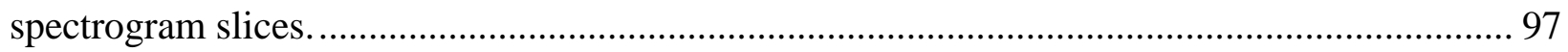

Figure 40 The average confusion matrix after training on linear Librosa spectrograms............. 98

Figure 41 The average confusion matrix after training on Mel Librosa spectrograms. .............. 99

Figure 42 The average confusion matrix after training on logarithmic Librosa spectrograms. . 100 


\section{TABLES}

Table 1 Dataset comparisons from Dai and Lui [18]....................................................... 28

Table 2 Results from three datasets trained using three different techniques by Costa, Oliveira,

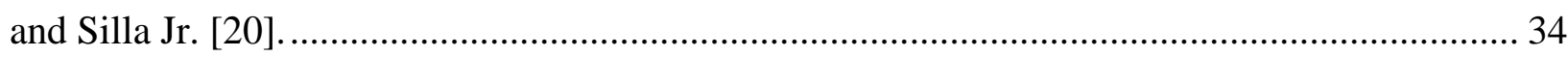

Table 3 Results from different data augmentation techniques used by [21].......................... 36

Table 4 Comparison of linear spectrograms, Mel spectrograms, and MFCC by [44]................ 39

Table 5 Songs per genre within Collection A's music library............................................... 44

Table 6 Songs per genre withing Collection B's music library........................................... 45

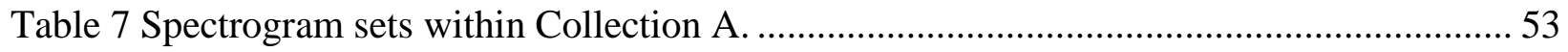

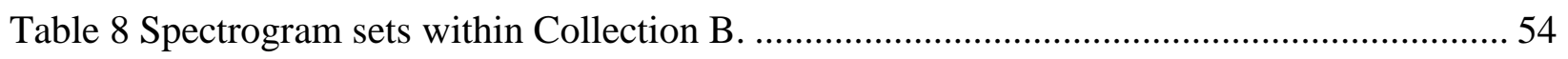

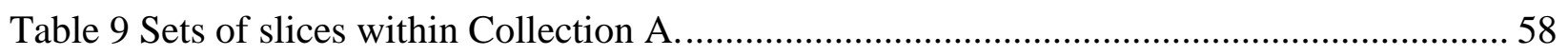

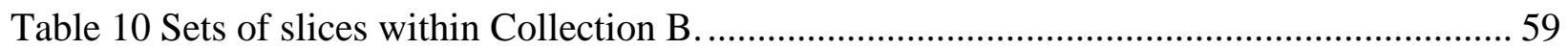

Table 11 Datasets created from Collection B while the number of slices per genre is set to a

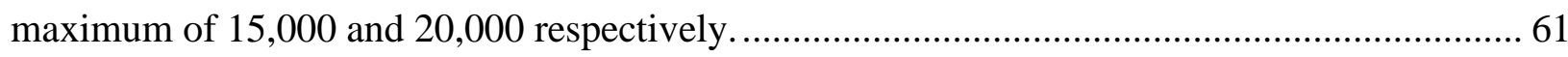

Table 12 The two datasets used to compare Collection A and Collection B directly. ................ 62

Table 13 A list of layers in the implemented CNN architecture ..............................................6 65

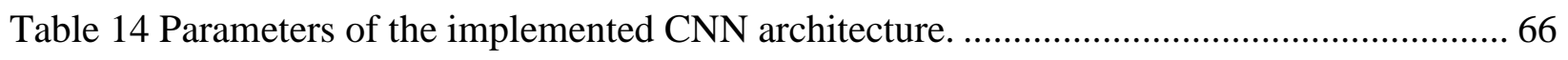

Table 15 Dataset extracted from Pre-Collection A used for testing different activation functions

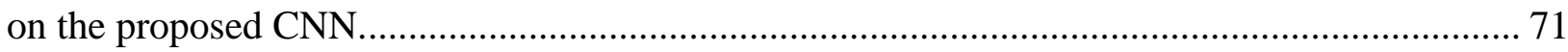

Table 16 Results from testing different activation functions on the proposed CNN.................. 72

Table 17 Datasets extracted from pre-Collection A used for testing varying lengths of

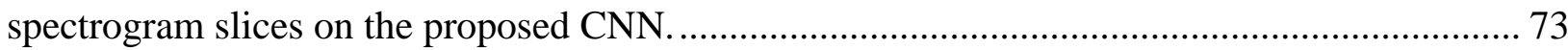

Table 18 Results from varying the length of spectrogram slices on the proposed CNN............ 73

Table 19 Results from testing different windowing functions on the proposed CNN. .............. 74

Table 20 Results from the change in the number of output classes....................................... 74

Table 21 Datasets extracted from pre-Collection A and Collection A used for testing the number

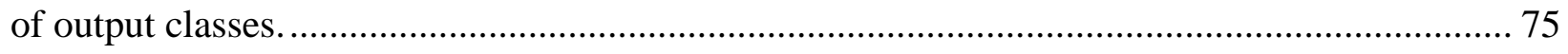

Table 22 Dataset extracted from Collection A for testing the number of hidden layers within the

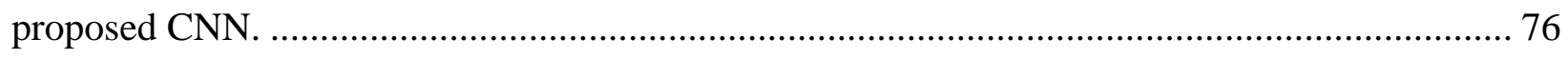

Table 23 Results from changing the number of hidden layers within the proposed CNN.......... 77 
Table 24 The two extracted datasets used to compare Collection A and Collection B directly... 79 Table 25 Results from comparing Collection A with Collection B using the proposed CNN. .... 80 Table 26 The datasets extracted out of Collection B used for comparing an unevenly distributed

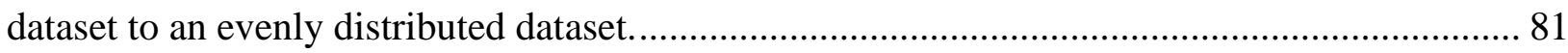
Table 27 Results from comparing an unevenly distributed dataset to an evenly distributed dataset, both extracted from Collection B..................................................................... 82

Table 28 Results from comparing linear spectrograms from SoX and Librosa. ....................... 83

Table 29 The dataset extracted from the linear Librosa spectrogram slices............................ 84 Table 30 Average accuracy and majority vote accuracy obtained used linear Librosa

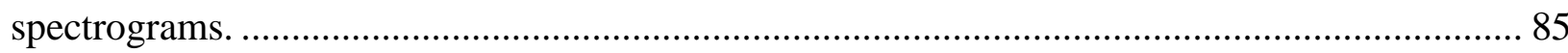

Table 31 The dataset extracted from the Mel scaled Librosa spectrogram slices. ..................... 88 Table 32 Average accuracy and majority vote accuracy obtained used Mel scaled Librosa

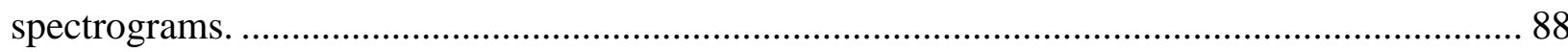

Table 33 The dataset extracted from the logarithmic Librosa spectrogram slices. ................... 91 Table 34 Average accuracy and majority vote accuracy obtained used logarithmic Librosa spectrograms.

Table 35 A comparison of accuracy and majority vote accuracy across linear, Mel scaled, and

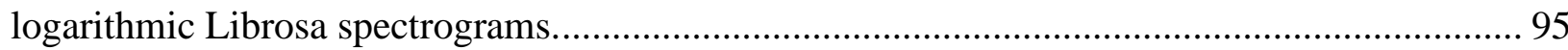

Table 36 Difference between training session accuracies for each spectrogram type................ 95

Table 37 The total slices by genre which are incorrectly labelled for the three types of spectrograms 


\section{ABBREVIATIONS}

AI

$\mathrm{AE}$

AUC-ROC

BN-LSTM

$\mathrm{CNN}$

CRNN

DFT

DSP

ELU

FCN

GAN

GRU

IndRNN

LBP

LMD

LSTM

MFCC

MIDI

MSD

Multi-DNN

$\mathrm{NN}$

ReLU

RH

RNN

ROC

$\mathrm{RP}$

SoX

SSD
Artificial Intelligence

Auto Encoder

Area Under the ROC Curve

Batch-Normalized LSTM

Convolutional Neural Network

Convolutional Recurrent Neural Network

Discrete Fourier Transform

Digital Sound Processing

Exponential Linear Unit

Fully Convolutional Neural Network

Generative Adversarial Network

Gated Recurrent Unit

Independently Recurrent Neural Network

Local Binary Patterns

Latin Music Database

Long Short-Team Memory

Mel Frequency Cepstral Coefficient

Musical Instrument Digital Interface

Million Song Dataset

Multilingual Deep Neural Network

Neural Network

Rectified Linear Unit

Rhythm Histograms

Recurrent Neural Network

Receiver Operating Characteristic

Rhythm Patterns

Sound eXchange

Statistical Spectrum Descriptors 


\section{INTRODUCTION}

\subsection{Motivation}

With the size of digital music collections growing steadily, organizing and classifying music within a collection has become essential for music retrieval. Music can be classified by genre, instruments used, mood description of the composition, and even whether a user might like a given song based on previous preferences. A common first step in structuring large collections of digital music for retrieval is to classify the music by genre label. Music genres are a categorical label created by humans used to describe the style of music [1]. There is no formal definition for musical genre labels as they are somewhat subjective but there is "perceptual criteria related to the texture, instrumentation, and rhythmic structure of music that can be used to characterize a particular genre" [2].

While the management of large collections of digital music commonly depend on correct genre labels, automatically classifying music by genre has also become an important task for music recommendation [3]. Automatic music recommendation systems have the ability to recommend new music to the listener based on personal preference. These systems have become an indispensable function in music software and the genre label has significant influence over the

music recommended to the listener [3]. The ability to automatically classify music by genre using machine learning can be quicker and arguably more accurate than doing it manually [4]. This thesis examines the difficult task of automatically classifying music into genres by analyzing the audio signal through image representations of its spectral domain. 


\subsection{Research Objectives}

The topic of automatic classification of music genre has been researched thoroughly over the years by using support vector machines or neural networks to classify the music. There are many areas within music classification that have been researched. One area focuses on different input formats which can be used for learning. Input formats such as raw audio, spectrograms, Mel Frequency Cepstral Coefficient, and MIDI (Musical Instrument Digital Interface) files have all been at the center of research in this area. This thesis concentrates on different types of generated spectrogram images that can be used to train a neural network. These spectrogram images represent a song's spectral content in the visual domain. The three types of spectrograms considered in this thesis are linear, Mel frequency, and logarithmic spectrograms. They all represent the same audio signal data but have different representations visually across the $\mathrm{Y}$ axis.

The linear spectrogram represents all frequencies equally along the $\mathrm{Y}$ axis. In contrast, the Mel scaled spectrogram puts more emphasis on the audio range which humans can differentiate more clearly. Humans can pick out differences between lower frequencies better than they can with higher frequencies [5]. For the human ear, the difference between a $200 \mathrm{~Hz}$ tone and a $300 \mathrm{~Hz}$ tone sounds more significant than the difference between $10,000 \mathrm{~Hz}$ and $10,100 \mathrm{~Hz}$ even though there is an equal amount of change in frequency. The Mel scaled spectrogram takes this into consideration by accentuating the lower frequencies on the $\mathrm{Y}$ axis rather than the higher ones. Finally, the logarithmic spectrogram simply shows the frequency data on a logarithmic scale along the $\mathrm{Y}$ axis. The nature of a logarithmic scale for frequencies from $0-22,050 \mathrm{~Hz}$ results in the bottom portion of the spectrogram being dedicated to the first $1,024 \mathrm{~Hz}$ while the top portion of the graph shows the rest of the frequency range $(1,024-22,050 \mathrm{~Hz})$. 
One objective of this research is to explore the differences between these three spectrogram types and how they affect the accuracy of a neural network music genre classifier. This leads to a better understanding of whether putting emphasis on different frequency ranges within a spectrogram while training a neural network is beneficial. There are several comparisons between different input formats in the current research literature; however, none were found that directly compared linear, Mel scaled, and logarithmic spectrograms.

In addition to observing the effects of changes to the input spectrogram type used for the network, other research objectives include the comparison of different neural network configurations and techniques of the classifier including the comparison of different dataset configurations. The composition of a dataset used for learning is important for a classification task so the neural network can correctly label never before seen data. The examination of commonly mislabelled data points within the testing dataset can also lead to insights on the performance of the classifier. Lastly, differing configuration values for the neural network such as the size of the dataset, number of genres for classification, and hyperparameters used to tune the network are used to evaluate their effects on genre classification accuracy.

\subsection{Thesis Contributions}

The research contributions of this thesis are:

- A proposed convolutional neural network which classifies music by genre using 2.56 second sections of songs converted into spectrogram images.

- The comparison and evaluation of linear, Mel scaled, and logarithmic spectrogram images as input for a convolutional neural network music genre classifier. 
- An assessment of architecture, hyperparameters, dataset parameters, and their effects on a convolutional neural network music genre classifier.

\subsection{Thesis Organization}

This thesis has been organized into five chapters which are as follows:

Chapter 1 includes an introduction and some background into music genre classification, the motivation behind the research, the objectives, and contributions made by this research.

Chapter 2 provides background knowledge behind music genre classification which starts with machine learning, neural networks and an introduction to a few different types of neural networks. This chapter goes into detail on how music genre classification has been solved using different types of neural networks, different types of datasets, and using different types of accuracy measurement techniques which have all had varying levels of success.

Chapter 3 explains step by step how to replicate the setup for the research done in this thesis. It starts with the gathering of music data, creating the spectrograms from the music, and extracting a dataset to train from. It outlines the type of neural network used and the hyperparameters used which includes the codebase and the hardware used. Lastly, it details the different types of accuracy measurements used in this thesis.

Chapter 4 contains the experiments conducted and the corresponding results obtained. Each subsequent experiment completed in this chapter builds off the prior experiments done to create the best fit network model. It then compares the accuracy from three different types of spectrogram datasets followed by an analysis of the mislabelled outputs of the network.

Chapter 5 gives a summary and conclusion from the research presented in this thesis. It also outlines areas for further research. 


\section{LITERATURE REVIEW}

\subsection{Machine Learning}

The terms Machine Learning and Artificial Intelligence (AI) are often used synonymously; however, machine learning is just one area of the much broader field of AI. Machine Learning is a way of analyzing observational data and automatically building a model based on the data [6]. The system can learn from the data and adapt if the data changes as opposed to a skilled programmer writing specific instructions and conditions to define the model [6]. It is "based on the idea that systems can learn from data, identify patterns and make decisions with minimal human interaction"

[7]. Machine learning has become very popular in recent years. Depending on the type of application and the dataset available, certain types of machine learning techniques are more appropriate than others for different applications.

There are generally four main types of learning algorithms in machine learning. The main types of learning algorithms include supervised learning, unsupervised learning, semi-supervised learning, and reinforcement learning [6]. Supervised learning utilizes a fully labelled dataset to build a mathematical model between the inputs and outputs of the dataset. Using the fully constructed model, supervised learning can be used to predict the output for new input data points [6]. With a dataset of credit card transactions, supervised learning can be used to predict future possibilities of fraudulent card transactions [8]. Whereas, unsupervised learning attempts to extract useful features from an unlabelled dataset without any specific target or goal in mind. Unsupervised learning can be used for clustering datasets. Unsupervised learning can be used to cluster transactional data to identify customer segments with specific attributes [8]. These customer segments could identify outliers within the segment which could point to transaction fraud [8]. Correspondingly, semi-supervised learning utilizes a dataset that contains both labelled and 
unlabelled data. Generally, a small amount of labelled data in combination with unlabelled data has been proven to produce considerable improvement in learning accuracy compared with unsupervised learning [9]. Finally, reinforcement learning takes a different approach through the use of a feedback mechanism. In reinforcement learning, the learning is accomplished through a process of being rewarded for correct actions or predictions. For example, reinforcement learning is often used in games where the intent is to minimize risk and maximize reward. Reinforcement learning could be applied to the game chess, where winning is rewarded and losing is penalized.

\subsection{Neural Networks}

Training a neural network $(\mathrm{NN})$ is a technique of machine learning that is generally effective at extracting critical features from complex datasets and deriving a function or model that expresses those features [10]. Most NNs utilize a training dataset to first train a model. After the model is trained, the NN can then be applied to new or previously unseen data points and classify the data based on the previously trained model.

\subsubsection{Architecture}

Neural networks are complex systems built from multiple layers of neurons, also called nodes. These nodes take in any number of weighted inputs, computes a summation of those weighted inputs, adds a bias, and then applies an activation function to yield an output. Figure 1 shows the equation used before applying the activation function. The value $\mathrm{Y}$ can range anywhere from $-\infty$ to $+\infty$.

$$
\mathrm{Y}=\sum_{i=0}^{n}\left(\text { weight }_{i} * \text { input }_{i}\right)+\text { bias }
$$

Figure 1 Equation used within a neural network node. 
To determine if the node should activate or not, the value of $Y$ from Figure 1 is sent through an activation function. Figure 2 illustrates the node in reference to the inputs, weights, activation function, and output.

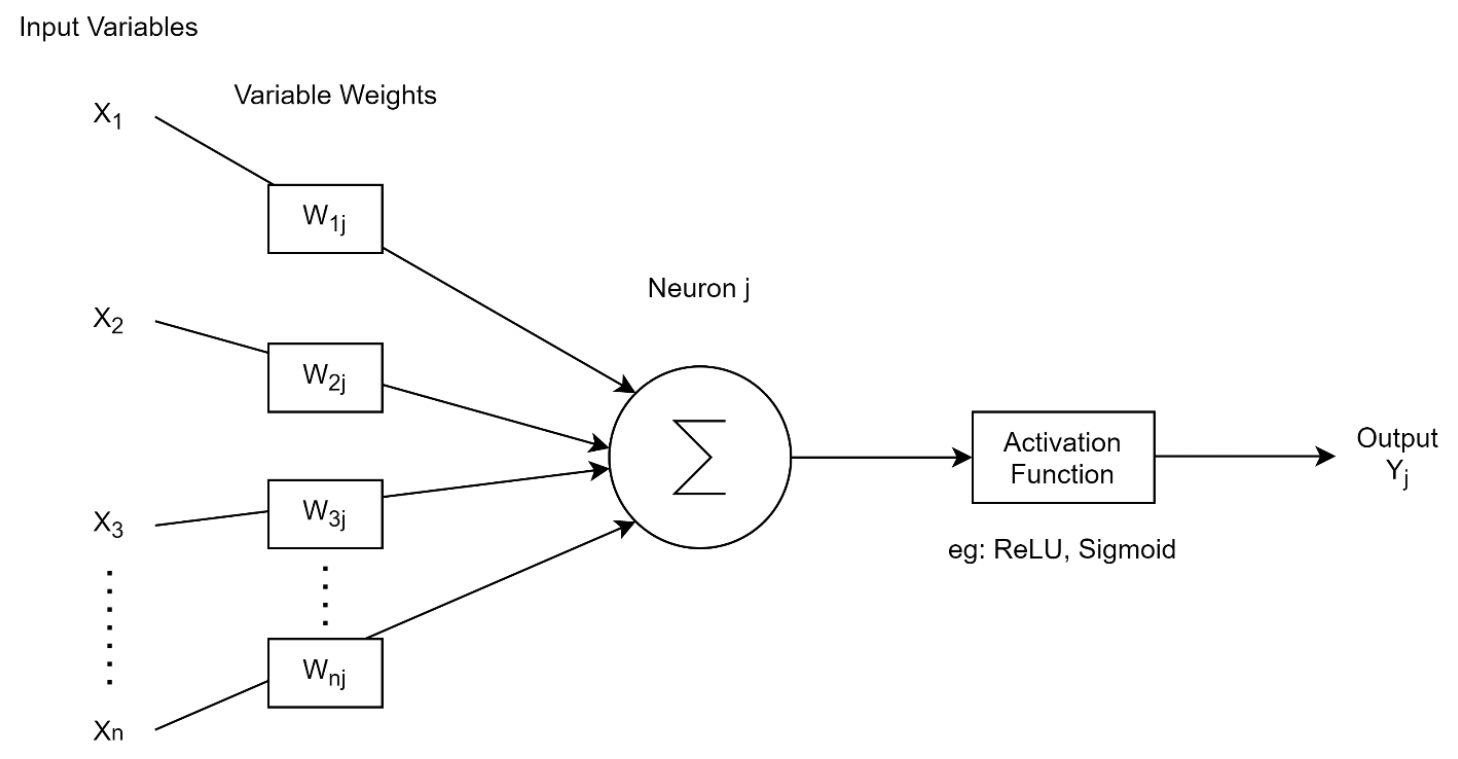

Figure 2 A neural network node diagram.

The output of the activation function, is then fed into the nodes of the next layer of the NN as input. This is depicted in Figure 3 where the output of hidden layer 1 is fed into hidden layer 2. 


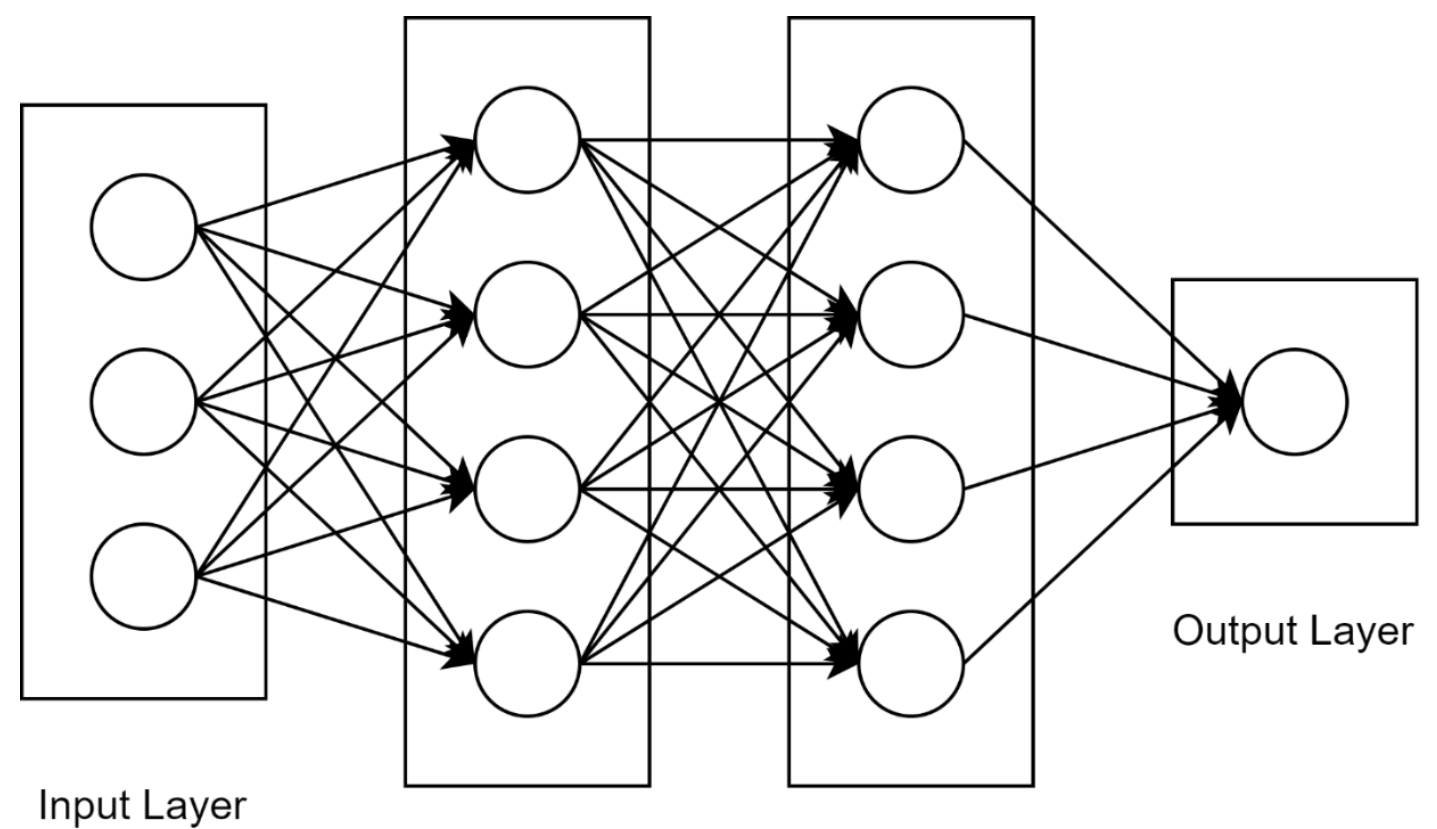

\section{Hidden Layer $1 \quad$ Hidden Layer 2}

Figure 3 A simple neural network architecture.

The activation function comes after the node's summation of weights and biases and will determine if the node should be activated or not. There are multiple different activation functions to choose from. Some common activation functions used are Sigmoid, Tanh, Rectified Linear Unit (ReLU), and Leaky ReLU which are all graphed in Figure 4. The properties of a Sigmoid function, which is graphed in the top left corner of Figure 4, will allow the node's output to take a value between 0 and 1 . In a classification problem with multiple output classes, the final layer nodes would each have different probabilities of activation. This will result in inputs being fed into the $\mathrm{NN}$, filtering through the layers of the network, and a certain number of output classes with different probabilities between 0 and 1 [11]. An example output of a neural network which classifies four classes could look like: [0.01578505, 0.00580563, 0.11662825, 0.86178107]. The highest value output node is the final predicted class of the NN for the respective inputs. For the example array, the fourth output class would be the final prediction. A similar situation would happen with any of the activation functions. 


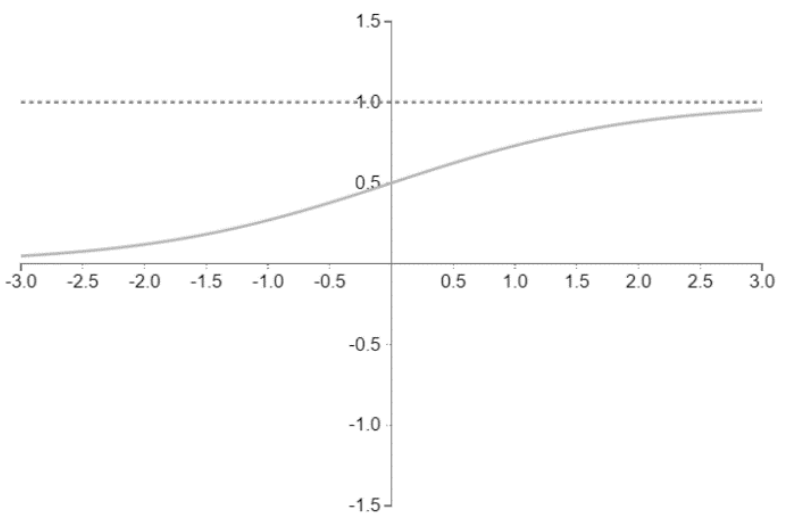

Sigmoid Function

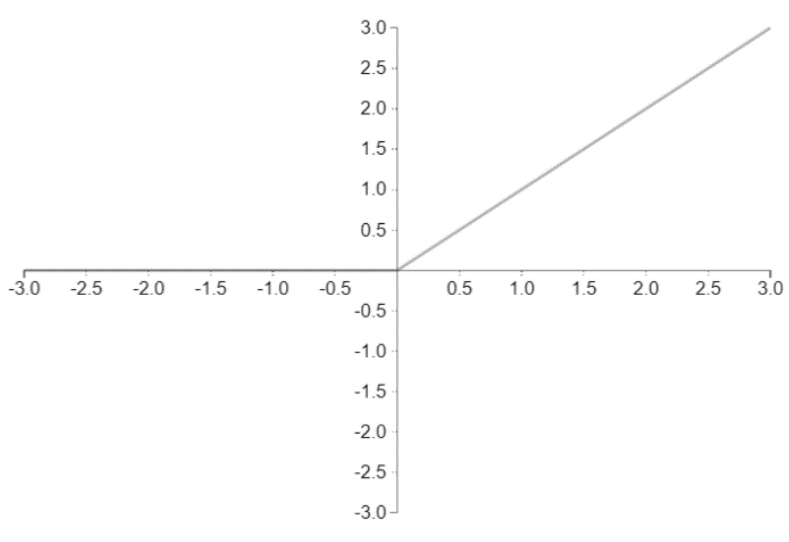

ReLU Function

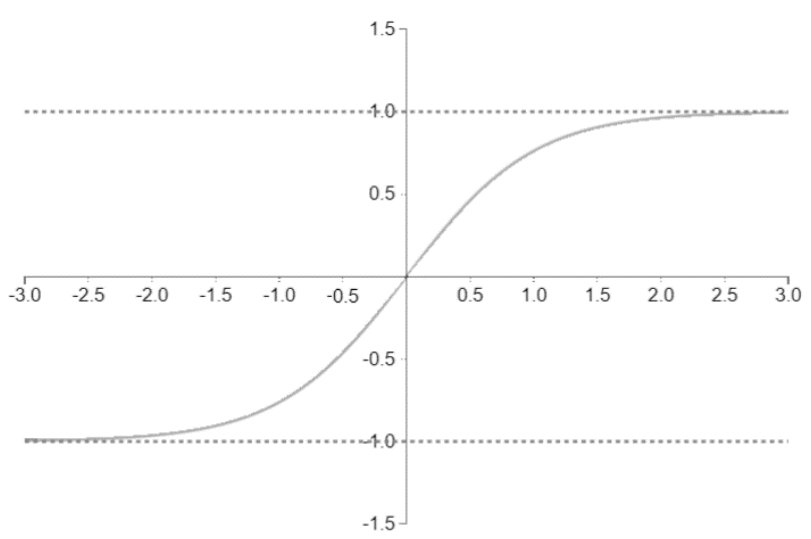

Tanh Function

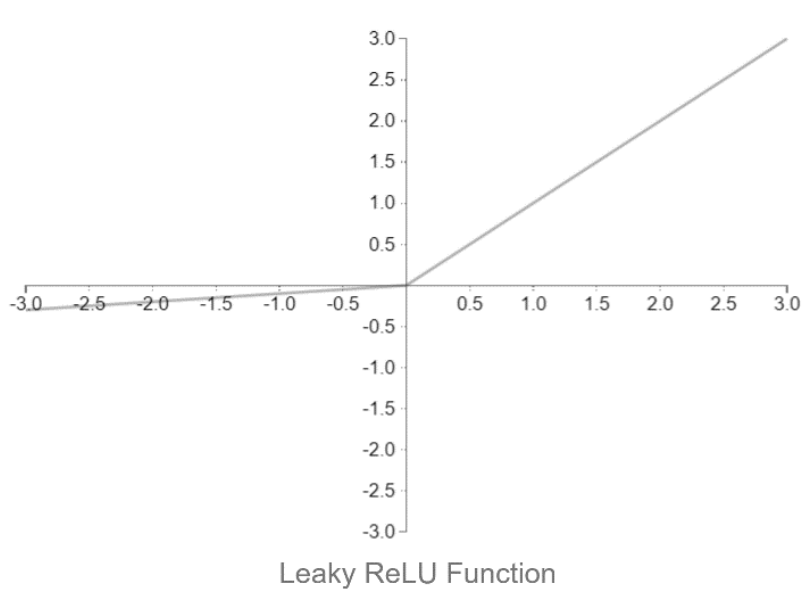

Figure 4 Different activation function graphs used by neural networks.

\subsubsection{Hyperparameters}

Before the training of the NN starts, there are several settings that one sets to control the behavior of the learning algorithm. These settings are called hyperparameters and generally are not adapted by the learning algorithm itself [11]. There can be hyperparameters set which determines the network structure like number of nodes within a layer and the number of layers within the network. There are also hyperparameters set to determine how the network is trained like epochs, batch size, learning rate, weight initialization, and dropout.

The main hyperparameters every network needs to be set prior to training are the number of epochs, batch size, and the number of batches or iterations. One epoch is when the entire dataset is passed forward and backward through the network once. One epoch is too big to feed the computer 
all at once so the dataset is divide into several smaller batches [12]. Batch size is the total number of training examples present in a single batch. The total number of iterations is the number of batches needed to complete one epoch [12]. For example, a dataset of 100,000 examples in it which are split into batches of size 500 would make 200 iterations or steps. Once the whole dataset of 100,000 examples have passed through the network, one epoch is finished.

Another very common hyperparameter that is required is the learning rate. This value is perhaps the most important hyperparameter because it controls the amount of change that happens to the model during each iteration [11]. In other words, the amount that the weights are updated during each iteration. If the learning rate is set too small the model will not learn quickly enough or may get stuck on a suboptimal solution [11]. If the learning rate is set too large, the model will learn quickly but at the cost of arriving on an optimal final set of weights. The model is likely to oscillate around the correct weights but achieve a suboptimal solution which will affect accuracy [11]. A perfectly configured learning rate will allow the model to learn quickly and arrive on the optimal set of weights in the number of training epochs used [13].

The way the weights and biases for each layer of nodes are initialized is considered a hyperparameter to set before training the NN. The initialized biases values can be set to 0 throughout the network without issue [11]; however, the aim of the initialized weights is to avoid exploding or vanishing gradients [14]. In the situation of exploding or vanishing gradients, the network is unable to create an optimal model for the training data [14]. If the initial values of the weights are too large this leads to exploding gradients and if they are too small it leads to vanishing gradients. Initializing weights with inappropriate values will lead to issues and to help with this, most machine learning libraries have functions available to use. A commonly used and 
recommended initialization is Xavier initialization which prevents the issues with gradients described above [14].

A final common hyperparameter is dropout. Dropout is a powerful regularization technique used to avoid overfitting [11]. To avoid overfitting, dropout improves generalization of NN's model which means the network can better classify newly introduced data. Dropout will essentially turn off or dropout a certain percentage of random nodes throughout a layer in the network. Dropout can be applied during training to any layer within the network other than the output layer. It will force the network to make new connections or create new paths through the network as it trains which makes the model more general and robust in terms of the problem it is trying to solve [15].

\subsubsection{Training}

A loss function, synonymous with cost function, is used to evaluate a set of weights and biases throughout the network during training. The accuracy of the NN increases if the cost function is minimized, which is typically defined as Mean Squared Error [16]. There are a number of different preprogrammed functions in most machine learning libraries to use for a cost function. Mean Squared Error is used in "regression problems where a quantity is predicted" [16]. This thesis focuses on a classification problem, which means cross-entropy will be used as the cost function. Cross-entropy is measured by calculating the error between two probability distributions [16]. Using cross-entropy as the cost function will seek a set of model weights that minimize the difference between the training data and the model's predictions [11].

Finding the global minimum for the cost function will determine the most accurate weights and biases throughout the network and can be found using gradient decent [17]. An example cost function is displayed in Figure 5. Since there are only two variables that the cost function depends on, $\mathrm{V}_{1}$ and $\mathrm{V}_{2}$ in the figure, it is visually easy for a human to determine the global minimum. It is 
simple and can effortlessly be graphed. Most NN cost functions depend on thousands of variables (weights and biases) which can not be visualized on a multi-dimensional graph. To compute the global minimum, a NN first starts computing the derivative of the cost function. After the derivative has been determined the slope of the cost function is known, and therefore the direction to move in order to approach the global minimum [17]. It uses the learning rate hyperparameter value as the size of step to take in the direction of the global minimum.

Each training step includes calculating the gradient and uses backpropagation to update the weights. Backpropagation involves taking the partial derivative with respect to each weight throughout the network to calculate how the weight should change. With backpropagation, the weights are updated in the NN so in the next forward pass, the NN should be more accurate. Gradient decent is "a powerful way of minimizing the cost function, and so helping the [neural network] learn" [17]. 


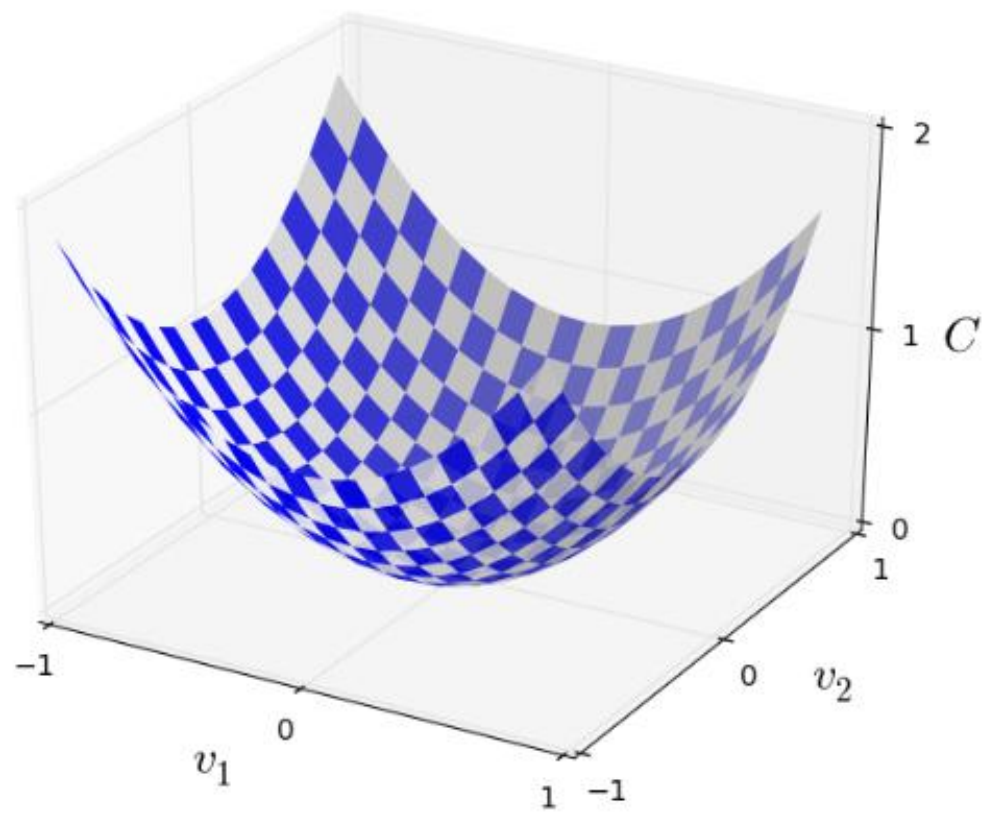

Figure 5 Example of a Cost Function (C) of two variables (v1, v2). Source [17].

\subsubsection{Accuracy Measurements}

After the $\mathrm{NN}$ has been trained, there is usually a measurement of accuracy to see if the $\mathrm{NN}$ is able to correctly label, generate, or classify the data. For classification problems the dataset being used is split up into training and test data. Training data is used to train the NN and after being trained, the test data is reserved to test the $\mathrm{NN}$ on data it has not previously been exposed to. The test data, like the training data, is usually labelled with the correct label in supervised learning scenarios. The accuracy of the network is measured by the number of data points within the test dataset which are classified correctly in comparison to their actual label. The accuracy is usually denoted in a percentage of accurate predictions. This thesis focuses on classification and thus test accuracy refers to the percentage of accurately predicted data points within the test dataset after the NN has been trained. 
Another similar measure of a NN's accuracy as utilized in this thesis is a majority vote accuracy. Majority vote accuracy is commonly found in music classification research [1, 3, 18-21]. In music genre classification, it is common to slice a song up into smaller sections and train on those smaller slices. For example, a 3:00 minute Pop song might be sliced into smaller, 2.5 second increments. The 3:00 minute song would then be represented as 72 data points within the training or test dataset and all 72 data points would be classified by the NN. The majority vote accuracy takes the mode of the predictions across the song's data points. For example, the 72 data points could be classified by the neural network as:

- 55 data points - Pop

- 10 data points - Rock

- 6 data points - Rap

- 1 data point - Classical

The normal test accuracy would be 55 accurate predictions out of 72 which is $76.39 \%$ accurate. By using a majority vote accuracy, classifying this song as Pop would be $100 \%$ accurate because the most common prediction for the song was Pop. The reasoning behind this type of measurement of accuracy is that "in real life, usually, we are interested in determining the genre of the whole music track (a clip of music), rather than the genres of the internal frames" [18].

\subsubsection{Confusion Matrix}

Another measurement of accuracy or performance used in classification is called a confusion matrix. This is a table of predictions versus actual labels on data points within the test dataset and allows for easy identification of confusion between class predictions. "The number of correct and incorrect predictions are summarized with count values and broken down by each class" [22]. A 
confusion matrix not only gives insight into the errors of predictions being made but more importantly the types of errors being made and the confusion the NN might be having between classes. An example of a confusion matrix for a classification problem with 10 classes is shown in Figure 6.

\begin{tabular}{|c|c|c|c|c|c|c|c|c|c|c|}
\hline & \multicolumn{10}{|c|}{ Predicted Labels } \\
\hline & 0 & 1 & 2 & 3 & 4 & 5 & 6 & 7 & 8 & 9 \\
\hline 0. & 167 & 0 & 0 & 0 & 0 & 0 & 0 & 0 & 0 & 0 \\
\hline 1 & 0 & 157 & 1 & 1 & 0 & 1 & 1 & 0 & 5 & 4 \\
\hline 2 & 0 & 3 & 163 & 1 & 0 & 0 & 0 & 0 & 0 & 0 \\
\hline 3 & 0 & 1 & 3 & 159 & 0 & 2 & 0 & 2 & 4 & 0 \\
\hline 4 & 0 & 1 & 0 & 0 & 165 & 0 & 0 & 3 & 1 & 3 \\
\hline 5 & 1 & 0 & 0 & 0 & 0 & 170 & 1 & 0 & 0 & 1 \\
\hline 6 & 2 & 1 & 0 & 0 & 2 & 0 & 165 & 0 & 0 & 0 \\
\hline 7 & 0 & 0 & 0 & 0 & 0 & 0 & 0 & 167 & 1 & 1 \\
\hline 8 & 1 & 10 & 1 & 3 & 0 & 2 & 3 & 2 & 142 & 2 \\
\hline 9 & 2 & 1 & 0 & 1 & 0 & 3 & 0 & 3 & 4 & 157 \\
\hline
\end{tabular}

Figure 6 Example of a confusion matrix.

The diagonal dark blue squares are the number of correctly predicted data points for each class. The other squares represent the number of times a prediction was incorrect per class. There is a square with 10 in the bottom left corner of the table. This specific square represents 10 data points which were incorrectly predicted as Class 1 because the actual label was Class 8. A confusion matrix can give more insight into the classification issues of the NN than the accuracy or majority vote accuracy can.

\subsection{Types of Neural Networks}

\subsubsection{Convolutional Neural Network}

A convolutional neural network $(\mathrm{CNN})$ is a type of neural network that is intended to process multi-dimensional vectors such as images [10]. A CNN can be used for both binary classification 
and multi-classification tasks where these classifiers differ only in the number of output classes. For example, a dataset of animal images can be utilized to train an image classifier. The CNN provided a vector of pixel values from an image that is accompanied with the correct output class label the vector describes (cat, dog, bird, etc.). When an image is provided as an input to the CNN for training, it will attempt to classify the image with an output class. For every training image, the classification is compared with the expected output class label. The weights throughout the network are then iteratively updated through a backpropagation process in an attempt to improve the accuracy of the CNN during training. Each iteration helps form the CNN model which defines the training dataset's features and its ability to correctly classify images.

\subsubsection{Generative Adversarial Network}

There are several other types of NNs that have been developed for different problem sets and application types [23]. For example, generative adversarial networks (GANs) have been used to generate brand new image content that have similar characteristics to a given training dataset [24]. GANs are composed of two NNs with conflicting objectives, data is feed from one network into the other creating a loop which forces each network to improve [24]. The first NN is the generator, which generates new sample data which is similar to real data. This sample data is then fed into the discriminator. The discriminator is the second $\mathrm{NN}$ which is previously trained on the real dataset. It is fed new samples from the generator where it tries to discriminate between the generated data and the real data [24]. This continuous loop results in the generator producing very realistic sample data. An example of this might be producing a realistic new image of a cat or even a human face.

\subsubsection{Auto Encoder}

Auto Encoders (AE) are another type of neural network which can be used to reduce the dimensionality of data into a smaller latent space while preserving imperative features. The input 
and output of an $\mathrm{AE}$ are both the same dimensions while the hidden layer, the middle layer of the network, is the compressed representation of the data [25]. Training an auto-encoder includes shrinking input down to a compressed representation and then decompressing the data with the aim to recover the original data [25]. Once trained, auto-encoders can be used to predict missing or corrupted values of images [23].

\subsubsection{Recurrent Neural Network}

Another common NN is the recurrent neural network (RNN). RNNs are used when sequential context is important. This means the output from a previous iteration may influence the current output [26]. Common application examples of RNNs are for text and speech data because the order of words is important with respect to the meaning of the sentence. RNNs can run into vanishing gradient problems while training [10]. The gradient is used to update the weights throughout the NN during backwards propagation. It shrinks across time which means long input sequences fed into the RNN will decay over time. This means RNNs can have short term memory issues. An example of an RNN displaying short term memory issues is if it could not distinguish that the cat was a female in the following sentence: "The cat was stuck in the tree for 24 hours until she was rescued". When the RNN comes across the word "she", all memory of the previous input word "cat" is forgotten. To solve this issue, long short-term memory (LSTM) networks can be used. LSTMs make use of gates which control the flow of information. These gates learn what information is important to keep and discard, which keeps the previous data relevant for the LSTM to train on [27]. 


\subsection{Formatting Music as Input into a Convolutional Neural Network}

\subsubsection{Spectrograms}

A spectrogram is a visual representation of sound as the frequencies vary in time. Often when a spectrogram image is generated the horizontal axis represents time, the vertical axis corresponds with frequency, and the amplitude of a particular frequency in time is represented by intensity or color in the image [28]. Figure 7 shows an example of this type of spectrogram. The spectrogram is created from 20 seconds of a song and plotted on a $0-22,050 \mathrm{~Hz}$ scale. The darker the pixels in the image, the greater amplitude in the song at that frequency.

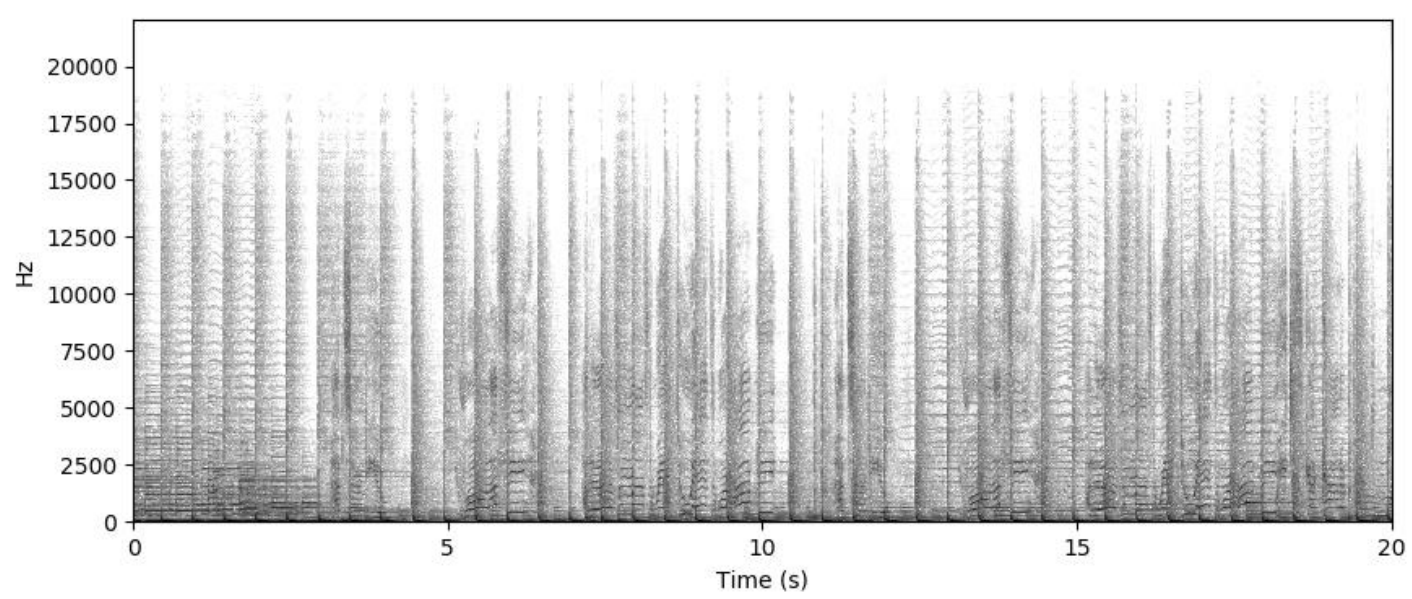

Figure 7 Example spectrogram plotted on a linear $Y$ axis of $H z$ and a $X$ axis in seconds.

A spectrogram represents a time domain signal in the frequency domain which for a digital time domain signal can be composed using the Discrete Fourier Transform (DFT) algorithm [29]. A finite number of sample frequencies are converted into a spectrogram image. To use the DFT algorithm, the sample frequencies must be sequential and equally spaced. The DFT algorithm is based on the idea of an infinitely long input, meaning the input will repeat over time infinitely [30]. When repeating the input signal over time, there will be discontinuities where the end of the signal is butted up against the beginning of the signal. For example, the point where the Nth signal ends 
and the Nth +1 signal starts, there will be discontinuity, displayed on the right of Figure 8 . This discontinuity affects the DFT algorithm and ultimately the spectrogram produced.
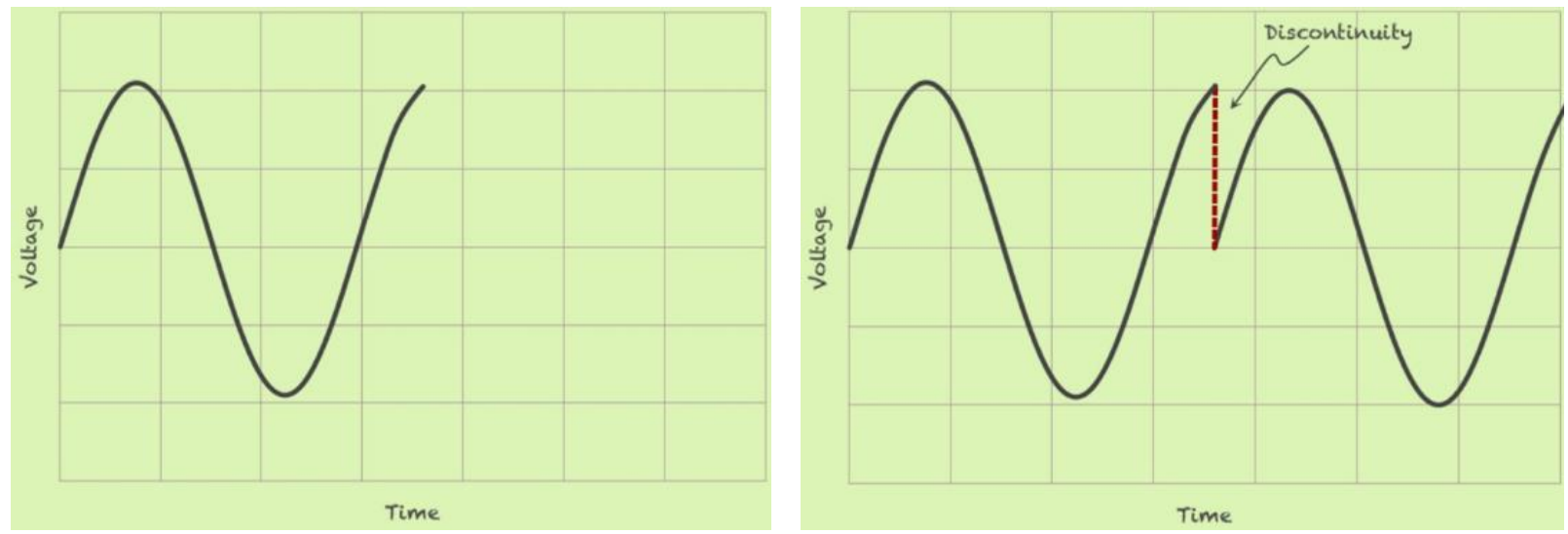

Figure 8 Example of a single signal, left; The signal being infinitely repeated over time and displaying a discontinuity, right. Source [30].

To deal with this issue, there are windowing functions which can be utilized to minimize this discontinuity. These windowing functions are a significant parameter to the Discrete Fourier Transform algorithm [31]. There are several windowing functions to choose from, including Hann, Hamming, Bartlett, Rectangular or Kaiser to name a few.

The Hann window "has good all-round frequency-resolution and dynamic-range properties" [31]. The Hamming window is known for better frequency resolution but lower dynamic range and for higher dynamic range but lower frequency resolution, there is the Kaiser window [31]. An example of how a windowing function works is in the left image of Figure 9 and on the right of Figure 9 shows how the window function helps minimize the discontinuity. 

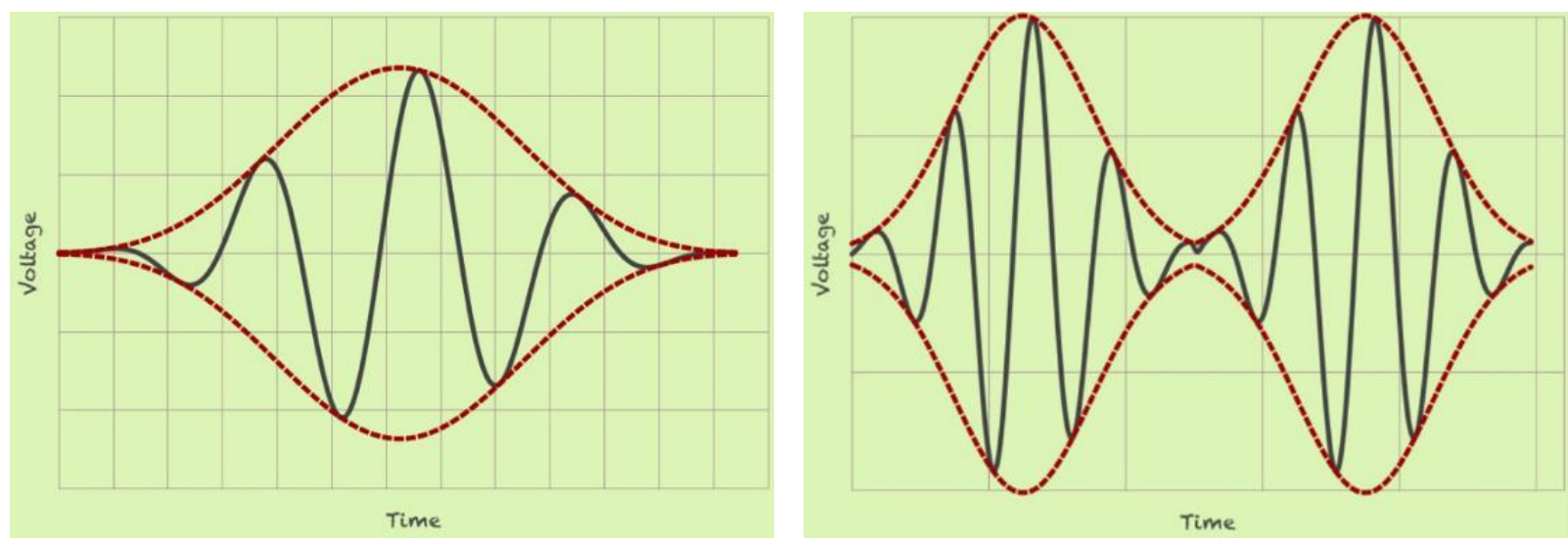

Figure 9 Window function, left; Window function repeated, right. This shows how a windowing function can help minimize the discontinuity. Source [30].

\subsubsection{Spectrogram Frequency Scale Visualizations}

Spectrograms can be visualized as in Figure 7 where the spacing of frequency is on a linear scale. Another way of plotting the frequency is through using a Mel frequency scale instead of linear frequency scale. The name $\mathrm{Mel}$ comes from the word melody which indicate the scale is based on pitch comparisons [32]. The Mel scale relates the perceived frequency of a tone to the actual measured frequency [33]. The scale is a non-linear transformation of the frequency scale [34]. It has been derived from experiments on human subjects to reflect more closely to what the human ear can differentiate [33]. The sole purpose of the scale is to describe the human auditory system on a linear scale. The formula [35] to convert Hertz (f) into Mels (m) is in Figure 10.

$$
m=2595 \log _{10}\left(1+\frac{f}{700}\right)
$$

Figure 10 Formula to convert Hertz $(f)$ to Mels $(m)$.

Spectrograms can also be plotted using a logarithmic frequency scale. In Figure 11 the same signal is plotted using a Mel scale, a logarithmic scale, and a linear scale. The linear scale does not put emphasis on any specific range of frequencies where as the Mel scale and the logarithmic scale emphasis $0-1,000 \mathrm{~Hz}$ in different ways. The emphasis on $0-1,000 \mathrm{~Hz}$ is highlighted in Figure 11. To plot the frequency scale in an image there needs to be a set height in pixels and then the frequency 
scale is partitioned into bins or bands. These are referred to as Mel bins if it is on the Mel scale, or a frequency bin if it is on a linear or logarithmic scale [34]. An image of 128 pixel height would have 128 frequency bins.

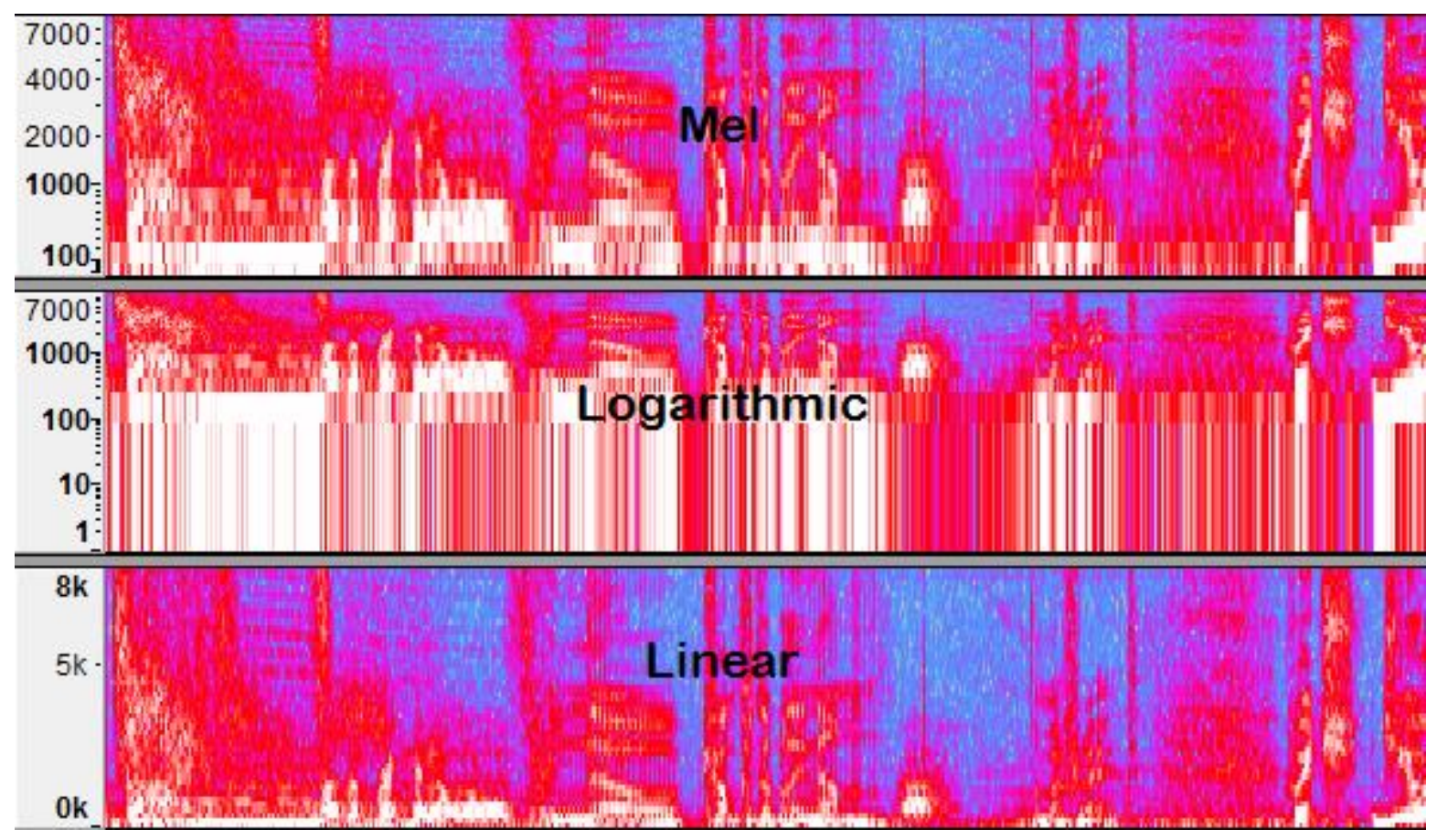

Figure 11 Using the same audio signal, the difference in frequency scale used to create a spectrogram is demonstrated. Source [32].

In the following chapters, unless otherwise stated, the spectrograms used are images with a horizontal time axis, vertical frequency axis, and amplitude of the frequency represented as black and white contrast in the image. The vertical frequency axis will vary from linear, Mel, and logarithmic but will always be specified.

\subsubsection{Mel Frequency Cepstral Coefficient (MFCC)}

In sound processing, the "Mel Frequency Cepstrum is a representation of the short-term power spectrum of a sound, based on a Mel scaled spectrogram" [36]. Mel Frequency Cepstral Coefficients (MFCCs) of an audio signal are a small set of features which concisely describe the overall shape of a spectral envelope [36]. The MFCCs are coefficients that make up the Mel 
Frequency Cepstrum [36]. The purpose of the MFCC is to adapt the audio signal feature to the human hearing system. Humans can hear frequencies below $1,000 \mathrm{~Hz}$ as a linear scale and frequencies above as a logarithmic scale [36]. For this reason, MFCC is prevalently used in speech recognition systems [36]. An example MFCC graph is in Figure 12. MFCCs are obtained by averaging spectrogram values over Mel frequency bands which improves stability, but also removes information. For this reason, MFCCs are effective at describing a signal over a short time window of less than $25 \mathrm{~ms}$. "Over time intervals larger than $25 \mathrm{~ms}$, the information loss becomes too important" [37]. For music genre classification, longer intervals help represent the song better. This means MFCC have a poor classification performance most of the time [18].

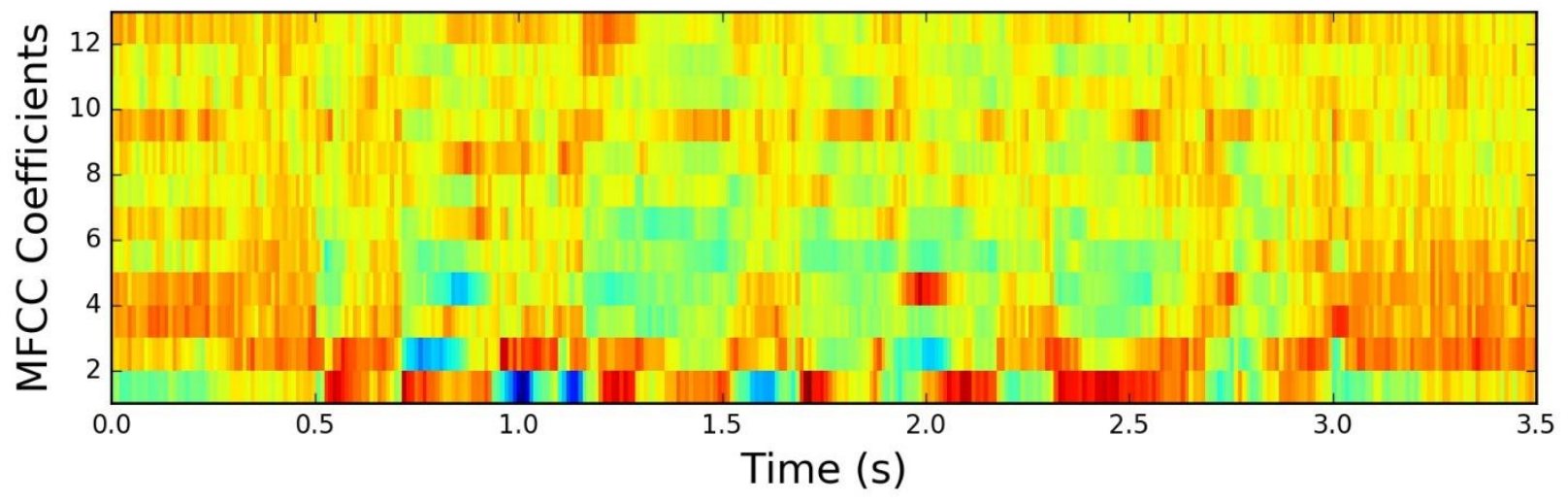

Figure 12 MFCCs extracted from an audio signal. Source [33].

\subsubsection{Scatter Transformation}

A scattering transform, like MFCCs, provides a low-level invariant representation of the signal [37]. Scattering transform is an extension of MFCC [37]. Anden and Mallat describe scattering transform as:

The information lost by mel-frequency spectrograms can be recovered with multiple layers of wavelet coefficients. In addition to being locally invariant to time-shifts, scatter transform is also stable to time-warping deformation. ... The computational 
structure is similar to a convolutional deep neural network, but involves no learning.

It outputs time averaged coefficients, providing informative signal invariants over potentially large time scales. [37]

\subsection{Music Genre Classification}

Classifying music is generally split up into two steps: feature representation and the classifier design [18]. A considerable number of research papers have been focused on classifying music genre of compositions. A large number of those papers concentrate on the representation of the features $[1,3,18-21,38-40]$, which generally aim to make the features more discriminative. The features extracted are often hand-crafted which requires in-depth domain knowledge and engineering ingenuity [41]. Hand-crafted typically refers to features which are manually engineered by the researchers. Another large section of music genre classification research draws more attention to the NN architectures and classification methods which exploit the advantages of different classifiers [18, 20, 40-43]. There are many network architectures that can classify music, choosing between the different types of NN architectures available usually depends on the type of input being used for the network. The architectures can vary from CNNs [19-21, 42-45] to RNNs $[3,40,43]$ and some researchers are still producing results with Support Vector Machines (SVM) $[1,20,38]$. The type of input also varies from paper to paper while authors are trying to decipher what the most promising input type is. Numerous input types have been used including spectrograms [20, 21, 42-45], MFCC and scatter transformation [3, 18, 19, 40, 44], and finally engineered or hand-crafted features $[1,20,38,39]$. The research in the following sections have been grouped according to the type of input utilize starting with a section for hand engineered features, then MFCCs, and finally spectrograms as input for the NNs. 


\subsubsection{Music Genre Classification Focused on Engineered Features}

Many machine learning methods used to classify music genre, such as SVMs [46] and Decision Trees [47], are trained on human engineered features extracted from the raw music file [3]. In this section, the research introduced focus on engineered features and feature representation. The human engineered features are constructed prior to the classification and machine learning implementation. Machine learning techniques are heavily reliant on the quality and accuracy of the features being used as input. The research presented in this section have a common goal of condensing the dataset by striping out non-essential data points while maintaining the critical information for the $\mathrm{NN}$ to learn from. The features extracted from the dataset should be comprehensive, compact, and effective; this means they should represent the music well, require a smaller amount of storage, and require little computation to extract [1, 48-50]. If the correct features are not extracted or there is any loss of data while extracting, the machine learning phase will lack the ability to make use of vital information and thus the features chosen will significantly affect the final results and accuracy of the work [40]. Deciding on the correct and pertinent features to extract from the dataset is difficult because it requires significant domain knowledge.

Humans are particularly good at listening to short samples of songs with the ability to distinguish the artist, the song title, and even the genre to a certain degree of accuracy. Emulating these abilities has been attempted through a number of $\mathrm{NN}$ approaches which have shown varying levels of success [20]. A popular application example for automatically determining both artist and song title from music data is the mobile device application Shazam. Shazam is primarily known for being able to determine the song title and the artist from only a few seconds of a song. Shazam has also been working on classifying different aspects of music data including genre, instruments used, mood description of the composition, and whether a given user might like a given song [39]. Shazam uses a technique in which they refer to as a song's signature. Shazam defines a song's 
signature as the large peaks in amplitude taken from the song's spectrogram. "It's like collecting the locations of the highest mountain peaks in a region; instead of (latitude, longitude, altitude), we have (time, frequency, amplitude) for these prominent peaks" [39]. O'Brien from Shazam put together a NN which consisted of two fully-connected layers with a final classification output layer of genre labels [39]. "This resembles a very "vanilla' multi-class classifier model” [39]. He achieved test accuracy in the low 90\% range. Combining his NN with another Shazam employee's track-level collaborative filtering features, he was able to slightly improve the model.

Lopes et al. [1] presented a method to limit the number of training data points to only include the ones that had shown better discrimination with respect to the output class. Their objective was "to build, from only a portion of the training data, a music genre classifier with at least similar performance as when the whole data is used" [1]. The dataset used consists of 900 songs split equally into 10 music genres from the Latin Music Database (LMD) dataset [1]. The 10 genres within the LMD dataset have significant similarities with regard to instruments, rhythmic structure, and harmonic content [38]. The LMD dataset is known for being particularly challenging to discriminate between the genres. The approach Lopes used was to create a 34-dimentional feature vector which was computed from the averages and variances of five 30 second segments around the middle of each audio file. Each music piece was then represented by 646 instances (i.e. data points in the dataset) [1]. In the paper by Lopes et al., a SVM was used as the classifier. The classification happens at an instance level, which means there was a classification for each of the 646 instances of one audio file. The final classification used a majority vote accuracy. An audio file was classified into the most voted class among its instances. Keeping in mind the challenging dataset, the final accuracy they obtained was $59.6 \% \pm 13.5$. 
Others have had success in music genre recognition by creating spectrograms and extracting features from the spectrograms. Costa, Oliveira et al. [38] worked on music genre classification using different types of inputs including spectrograms. Using 900 songs from the Latin Music Database, they divided them into 10 music genres equally. From each of the 900 songs, they extracted three 30 second segments from the beginning, middle, and end of the songs. From the spectrograms they mathematically extracted several features where each 30 second spectrogram was represented as ten 28 -dimensional feature vectors. This ultimately resulted in 30 vectors for a single music piece. With those vectors as inputs, they trained an SVM and then compared their work with Lopes et al. [1]. Lopes had previously presented a method to limit the number of training data points to only include the ones that had shown better discrimination with respect to the output class. The final accuracy Lopes achieved was $60 \%$ whereas Costa, Oliveira et al. saw an improvement of $7 \%$ having achieved a $67 \%$ recognition rate.

\subsubsection{Music Classification using MFCC or Scatter Transformation}

In the previous section, the engineered features described by the research required significant domain knowledge to decide on the correct and applicable features to extract from the data. In this section, the research introduced also focus on feature representation; however, the features used as input into the neural networks are MFCCs or scatter transformation images. With these types of feature representation, less domain knowledge is needed because the features can be extracted using different libraries of code $[29,31,32]$. The author can then focus on different $\mathrm{NN}$ architectures or classifier designs instead of extracting relevant data from the song. These common features being extracted by libraries enable the machine learning algorithms to "extract and organize the discriminative information from the data" [20]. There is also less risk of striping out crucial information prior to training or having data loss issues in the preprocessing phase because the NN extracts the discriminative information from the dataset itself during training. This means the NN 
must do some of the features extraction itself to focus on the important data in the dataset and ignore the trivial data. NNs are exceptionally good at doing this feature extraction in the deep layers of the network which means humans do not need to preprocess the data before feeding it into the NN. The features extracted by the $\mathrm{NN}$ often perform better than those manually engineered features, which makes the final classification result better [3].

Dai and Liu [18] employed a multilingual deep neural network (Multi-DNN) to classify music genres. A Multi-DNN, has been widely used for automatic speech recognition with low resources [18]. Low resources in this context refers to the dataset, this approach can utilize a large dataset for training if a similar but smaller dataset does not have enough data to train a NN with. Both the larger and smaller dataset do not need to have the same number of outputs, in this case genres. The two datasets Dai and Liu used were the ISMIR dataset and the GTZAN dataset. Both are freely available [51, 52]. ISMIR consists of 1,458 music pieces [20] which is about 100 hours of song data across 6 genres and is significantly larger than the GTZAN dataset which consists of 10 hours of song data across 10 genres. The GTZAN dataset was initially collected by Tzanetakis [53] in 2000-2001 and has been used by several papers in the music field to measure different aspects of performance. The GTZAN dataset consists of 1,000 clips of 30 second duration, all divided evenly between 10 genres: Blues, Classical, Country, Disco, Hip Hop, Jazz, Metal, Pop, Reggae, and Rock [53]. An outline of each of the datasets can be found in Table 1. 
Table 1 Dataset comparisons from Dai and Lui [18].

\begin{tabular}{ll||ll} 
GTZAN & & ISMIR & \\
\hline Genre & Tracks & Genre & Tracks \\
\hline Blues & 100 & Classical & 640 \\
Classical & 100 & Electronic & 229 \\
Country & 100 & Jazz/Blues & 52 \\
Disco & 100 & Metal/Punk & 90 \\
Hip Hop & 100 & Rock/Pop & 203 \\
Jazz & 100 & World & 244 \\
Metal & 100 & & \\
Pop & 100 & & \\
Reggae & 100 & & \\
Rock & 100 & Total & 1,458 \\
\hline Total & 1,000 & & \\
\hline
\end{tabular}

To deal with the uneven output classes between the datasets, Dai and Liu "utilized the nearest neighbor algorithm to find 'the most similar resource' data from [the] large similar database" [18]. 'The most similar resource' was then adopted for the training of the Multi-DNN. Dai and Liu used the LSMIR dataset to train their Multi-DNN using scatter transformation. With their trained MultiDNN they used the GTZAN for testing purposes and they were able to achieve $93.4 \%$ accuracy. Their accuracy was a majority vote of all the frames in a music clip. Their reasoning behind reporting this type of accuracy was because "in real life, usually, we are interested in determining the genre of the whole music track (a clip of music), rather than the genres of the internal frames" [18]. From a graph found within the paper, Figure 13, the highest accuracy before the majority vote seemed to be $\sim 85 \%$ accurate. 


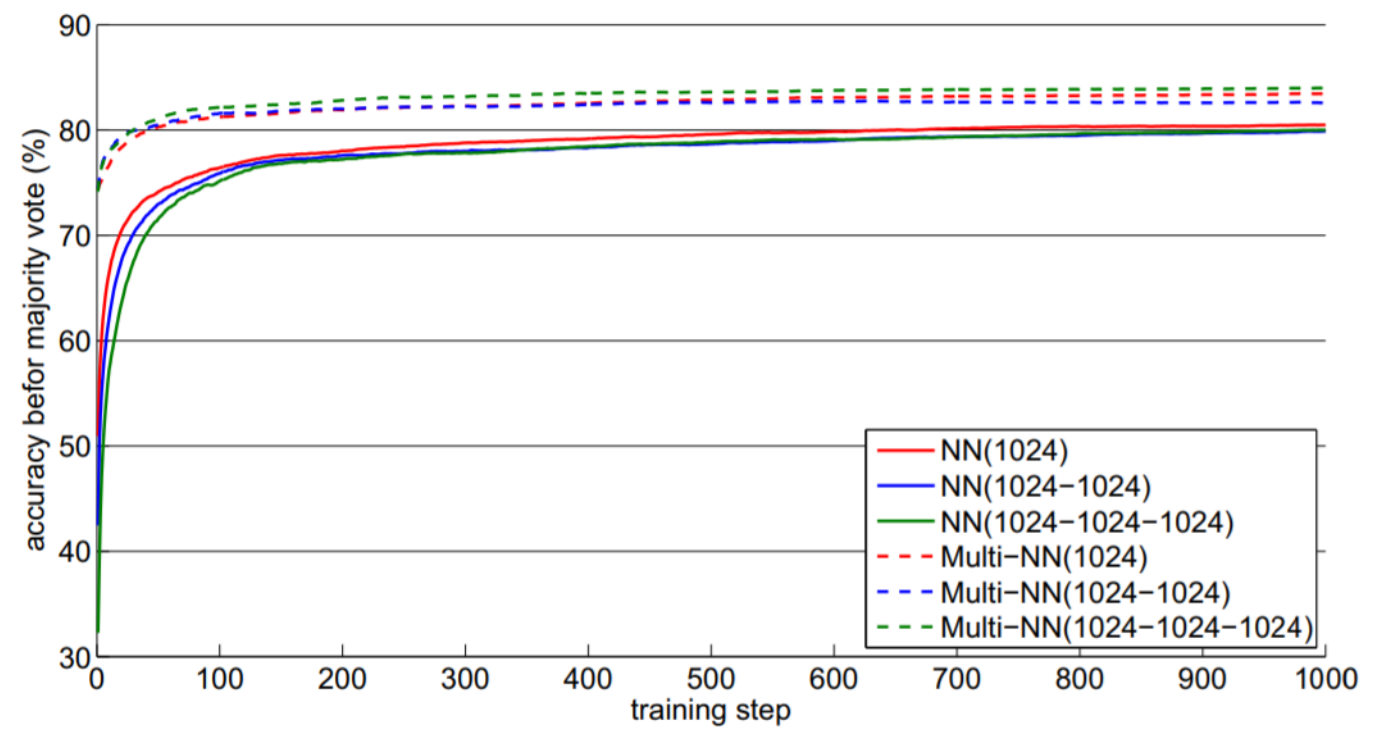

Figure 13 Accuracy before the majority vote from Dai and Liu. Source [18].

Wu, Han, Song et al. [3] implemented an Independently Recurrent Neural Network (IndRNN) to label music with its respective genre. An IndRNN has a longer memory than the popular LSTM and the Gated Recurrent Unit (GRU) thus it is a more suitable NN architecture for music labelling [3]. The length of the processable sequence for an LSTM is less than 1,000 inputs whereas for an IndRNN it is greater than 5,000 inputs [3]. Each neuron within the IndRNN "can independently process a time and space model" [3]. In their data preprocessing phase, prior to the IndRNN model, scattering transform was used which kept the data loss at a minimum. Wu, Han, Song et al. trained their IndRNN on the GTZAN dataset. Wu, Han, Song et al. achieved a high recognition rate using the GTZAN dataset and compared their results to other advanced models. The accuracy presented by $\mathrm{Wu}$, Han, Song, et was a majority vote where they achieved $96 \%$, which can be compared to the next best model mentioned in the paper by Dai and Liu [18] at 93.4\% accuracy.

Song, Wang, Han et al. [40] used a deep recurrent neural network to auto-tag music by genre, instrument, mood and so on. In the paper, there were numerous models tested including LSTM, GRU, and Batch-Normalized LSTM (BN-LSTM). Along with changing the model, the number of 
hidden layers in each network was tested with 4-6 layers, each containing 256 or 512 nodes in each hidden layer. The MagnaTagATune dataset was used which contains 25,863 clips of 29 seconds each "annotated with 188 different tags including genre, instrument, mood" [40]. By applying a filter of the top 50 tags, they were left with 21,108 clips to train and test their proposed system on.

The MagnaTagATune dataset creates complexity while calculating the accuracy of the work because there can be numerous tags associated to each clip. This means "most of the tags are false (0) for most of the clips" in the dataset [43]. This makes a percentage accuracy calculation inappropriate as a measure of performance of the network. Therefore, Area Under the ROC (Receiver Operating Characteristic) Curve (AUC-ROC) is used for determining the accuracy [40, 43-45]. "It is worth noting that a random guess is expected to score an AUC of 0.5 while a perfect classification 1.0, i.e., the effective range of AUC spans between [0.5, 1.0]" [44].

The proposed system by Song, Wang, Han et al [3] uses scattering transform to reduce the size of musical data while still retaining the relevant information. The model proposed was a five-layer RNN using GRU. After numerous tests based around the NN type, the number of hidden layers, number of nodes in the hidden layers, and the preprocessing of the dataset they received an AUCROC score of 0.909 using scattering transform, 5 hidden layers, and 512 nodes per layer. Other papers in the next section also use AUC-ROC to measure the performance of their network because they also use the MagnaTagATune dataset.

Vishnupriya and Meenakshi [19] calculated two types of feature vectors which were the MFCC and Mel Frequency spectrum. They utilized the dataset Million Song Dataset (MSD) which consists of audio tracks totalling $280 \mathrm{~GB}$. Vishnupriva and Meenakshi used 1,000 songs across 10 genres for their dataset. Feature extraction was done using the python based Librosa package [29] which has functionality available to calculate the MFCC of a song file. Librosa helped in providing good 
parameters for the network training. Afterwards, they applied a CNN to classify the music feature vectors into 10 different genre classes and compared the results between the two feature vectors extracted - MFCC and Mel Frequency Spectrum. The feature vector size for Mel Frequency spectrum was $599 \times 128 \times 2$ and for MFCC it was $599 \times 13 \times 5$ which is about a quarter of the size of the Mel Frequency spectrum vector. Vishnupriya and Meenakshi [19] split the dataset up into $80 \%$ training and $20 \%$ testing data which totalled 800 songs for training and 200 songs for testing.

The accuracy obtained in their paper using Mel Frequency spectrum feature vector was $47 \%$ and using the feature vector defined as MFCC, accuracy was 76\% [19]. Both of these accuracies were using a majority vote on the songs. MFCC has a better accuracy which was interesting granted the input into the $\mathrm{CNN}$ to obtain those results was about one quarter of the data to obtain an accuracy of $47 \%$ using Mel Frequency spectrum.

\subsubsection{Music Classification using Spectrograms}

The prior two sections have covered two different approaches to feature extraction for a NN trying to classify genres of songs. The first section covered hand engineered features which involves significant domain knowledge in order to extract the relevant data and discard trivial data from a song. The final results and accuracy of the work is dependent on the quality of the features extracted. A lack of vital information, like loss of data while extracting features, will negatively affect the machine learning accuracy [40]. The next section involved extracting features from songs using code libraries which leaves the in-depth domain knowledge up to the experts writing the library functions. Authors can be confident that crucial information and features of the song are being preserved by using a library to extract common features, for example MFCCs or scatter transformation. In this section, the research presented use code libraries to extract spectrograms as input into the NN. The spectrograms used can vary in the size of the images, color of spectrograms, and the scale of the axes used. Using spectrogram images as input for a $\mathrm{NN}$ is similar to using 
MFCCs because it allows the NN to do some of the feature extraction during training instead of during the preprocessing phase which happens while using hand engineered features. NNs excel at separating the critical data from the inconsequential data which often helps the performance in comparison to manual engineered features [3].

Costa, Oliveira, and Silla Jr. [20] investigate whether is it more beneficial to train an SVM classifier on acoustic features, train an SVM classifier on hand-crafted textural features extracted from a spectrogram, or finally to train a CNN on the actual spectrograms themselves to classify genre. Figure 14 shows these different classification paths investigated. Costa, Oliveira, and Silla Jr. used three different databases in this paper, Latin Music Database (LMD), ISMIR, and the African music database. The first two databases were classified by genre; however, the African music database was classified into different categories such as country, function, ethnic group, or instrumentation. All accuracies from this work were presented through a majority vote accuracy.
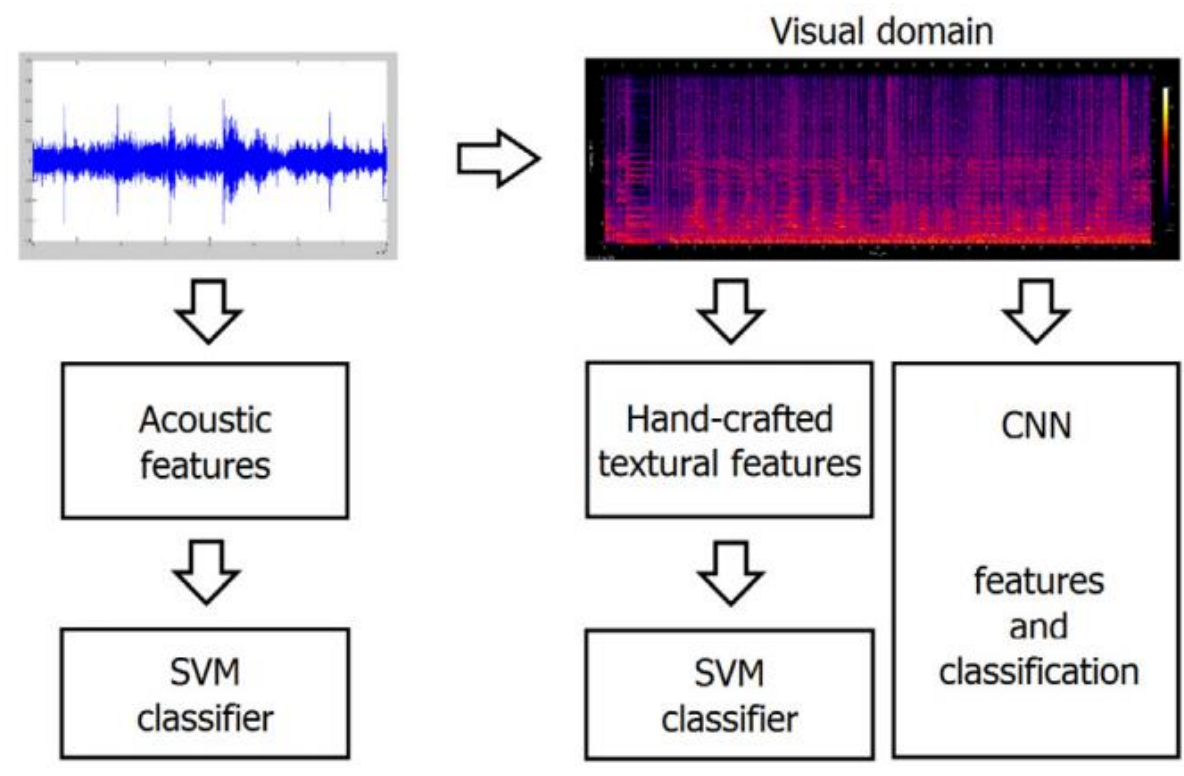

Figure 14 An overview of the classification options investigated in [20]. Source [20].

The acoustic features used to train the first SVM classifier included Rhythm Patterns (RP), Statistical Spectrum Descriptors (SSD), and Rhythm Histograms (RH). Next, the hand-crafted 
textural feature used to train the second SVM classifier was a variation of Local Binary Patterns (LBP) called Robust LBP. Finally, to create the spectrograms used for both the second SVM classifier and the CNN, Costa, Oliveira, and Silla Jr. used a single 60 second music segment from 0:30 to $1: 30$ of the original track. Depending on the database the sampling rate would change, LMD is sampled at $22,050 \mathrm{~Hz}$ and ISMIR is sampled at $44,100 \mathrm{~Hz}$, but each spectrogram produced would be created using a "window size of 1,024 samples using a Hann window function which has good all-round frequency-resolution and dynamic-range properties" [20]. After creating the spectrograms, the images were $800 \times 256$ pixels. To feed these spectrograms into the CNN, they were divided into slices with a size of $16 \times 256$ pixels. After creating the acoustic features, the hand-crafted textural features (visual features), and the spectrograms on each of the three datasets, they trained the respective SVM classifiers and CNN model on the inputs.

The Latin Music Database was used to train the first SVM classifier on the acoustic features and resulted in 52-67\% accuracy. It was used to train the second SVM classifier on the visual features extracted from the spectrograms which resulted in $87 \%$ accuracy. It was finally used to train a CNN using the raw spectrograms producing $83 \%$ accuracy. With the Latin Music Database, it was clear the visual features which were extracted from the spectrogram resulted in the best accuracy.

The ISMIR dataset was used next and trained using the acoustic features resulting in $63-76 \%$ accuracy. Using the visual features an accuracy of $83 \%$ was obtained and finally the CNN training on the spectrograms produced $86 \%$ accuracy. For the ISMIR dataset, the spectrograms feed into the CNN generated the best results.

The African Music Database was the last experiment they tried. With the acoustic features produced $64-87 \%$ accuracy and the visual features produced $88 \%$ accuracy. The CNN trained off 
spectrograms produced the best results at $94 \%$. Table 2 displays these results in a simpler manner from the past three paragraphs.

Table 2 Results from three datasets trained using three different techniques by Costa, Oliveira, and Silla Jr. [20].

\begin{tabular}{llll} 
Dataset & Features & & Majority Vote Accuracy (\%) \\
\hline LMD & Acoustic & RH & $52.0 \%$ \\
& & RP & $67.0 \%$ \\
& & SSD & $63.3 \%$ \\
& Visual & RLBP & $\mathbf{8 7 . 4 \%}$ \\
& & CNN & $83.0 \%$ \\
\hline ISMIR & Acoustic & RH & $63.4 \%$ \\
& & RP & $73.5 \%$ \\
& & SSD & $76.8 \%$ \\
& Visual & RLBP & $83.4 \%$ \\
& & CNN & $\mathbf{8 5 . 9 \%}$ \\
\hline Dfrican Music & Acoustic & RH & $64.1 \%$ \\
& & RP & $80.2 \%$ \\
& & SSD & $87.1 \%$ \\
& & RLBP & $88.2 \%$ \\
& Visual & CNN & $\mathbf{9 3 . 9 \%}$ \\
\hline
\end{tabular}

It is well known that deep learning systems are more difficult to train than shallow ones because they require more samples to provide a good classification model [21]. The authors from the previous paper continued their research into $\mathrm{CNN}$ learning using raw spectrograms in the work presented in [21]. They were specifically investigating whether data augmentation can improve the accuracy of their music genre classification using CNNs. The reason behind this research was because "well-labelled large datasets are not always available or they might be hard to acquire and to operate" [21].

Costa, Oliveira, and Silla Jr. used the same approach as they did in [20] where the spectrograms were divided up into slices and the slices were used as input into the CNN. The LMD dataset was 
used which is comprised of only 900 samples categorized into 10 genres. The data augmentation techniques they used included "adding noise, pitch shifting, time stretching, and loudness variance" [21]. They mention that not all data augmentation techniques were considered, for example rotation of the images, since that would distort important information across the time domain (X axis). An example of these different data augmentations is shown in Figure 15 which shows the same spectrogram slice that has gone through the different data augmentations.

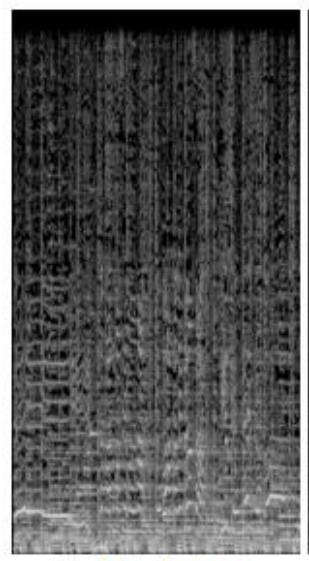

(a) Original sample.

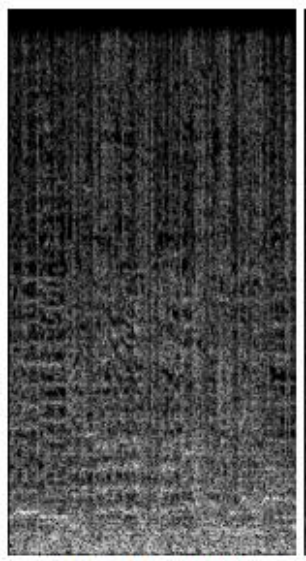

(f) Noised sample.

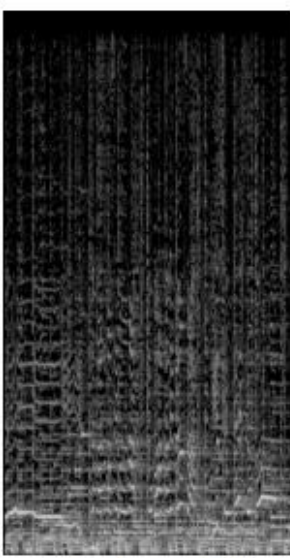

(b) Loudness $-10 \mathrm{~dB}$.

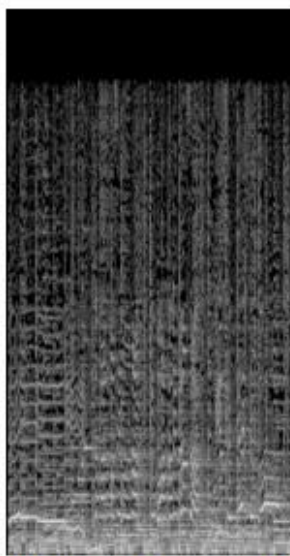

(g) Pitch Shifting - one tone (h) Pi lower. lower.

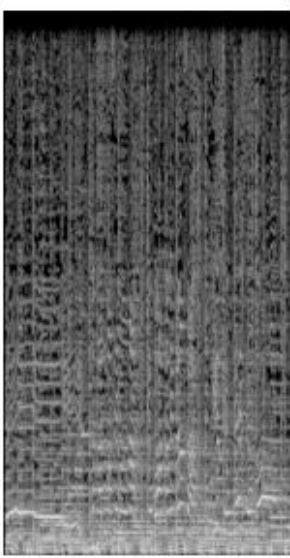

(c) Loudness $+10 \mathrm{~dB}$

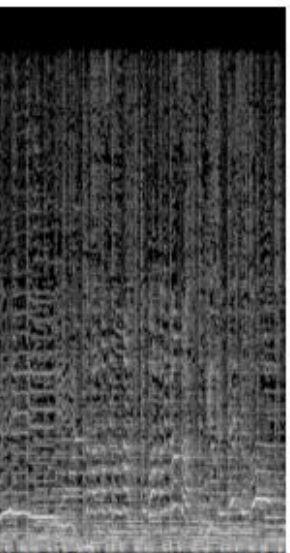

lower. higher.

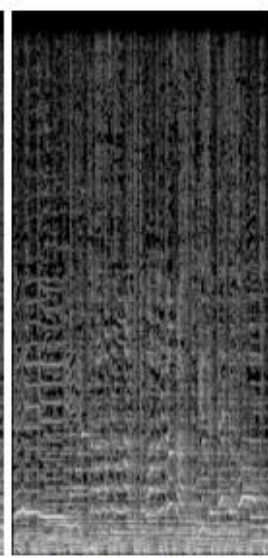

(d) Time stretching shorter spectrogram.

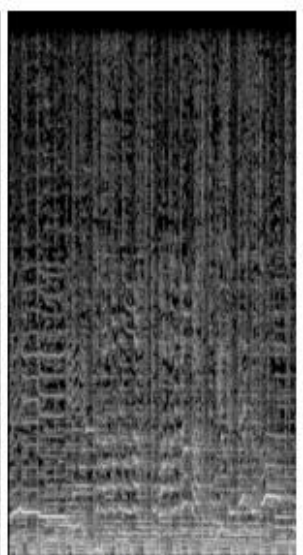

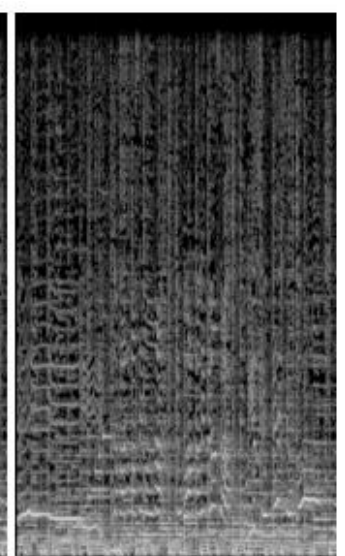

(e) Time stretching - longer spectrogram.

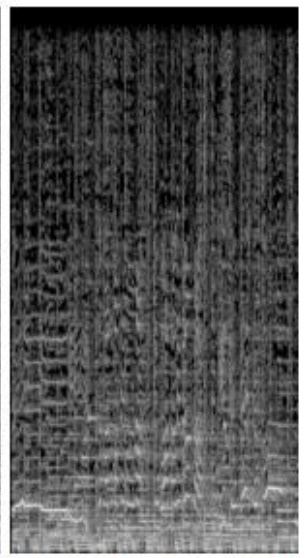
higher.

Figure 15 Data augmentation techniques done on a spectrogram slice by [21]. Source [21].

After an analysis of their results shown in Table 3, they concluded the data augmentation had a positive impact on their CNN. They indicate that with almost every data augmentation technique they saw an accuracy increase. Pitch shifting with one tone showing significantly "better results 
than all the other approaches with the exception of the other pitch shifting strategy with half tone" [21]. They corroborate their results with some other related audio work. In a separate paper that Costa, Oliveira, and Silla Jr. [21] found, noise addition did not improve the computational results. As well, in another paper presented by [21] it was "found that the use of pitch shifting, as a data augmentation strategy, provided the best results when compared to other strategies" [21].

Table 3 Results from different data augmentation techniques used by [21].

\section{Experiment description}

Baseline

Noise Addition

Loudness

Pitch shifting (Half tone)

Pitch shifting (One tone)

Time stretching

\section{Majority Vote Accuracy (\%)}

$86.3 \%$

$83.8 \%$

$86.4 \%$

$88.3 \%$

89.5\%

$86.8 \%$

Similar to Costa, Oliveira, and Silla Jr. in the previous two works, Despois [42] also trained a convolutional neural network on raw spectrograms. One difference to note is Despois worked with a personal music library consisting of 2,000 songs labelled with genre. The songs in the personal library were classified into a large number of genres and there were too few examples per genre to train on [42]. Despois reduced the number of genres by grouping the sub-genres into the main genre classes. By doing this the number of genres were reduced to a core six which included Hardcore, Dubstep, Electro, Classical, Soundtrack, and Rap. An example of this would be classifying Hark Rock, Metal, and Heavy Metal into one genre labelled Metal.

Now with a library consisting of six core genres, Despois converted each song into a spectrogram using SoX (Sound eXchange) [31] command-line music application utility which produced an image with a height of 128 pixels and length dependant on the length of song. After each spectrogram was produced, they were divided up into $128 \times 128$ pixel images which were referred 
to as slices or snippets. Each pixel along the $\mathrm{X}$ axis was $20 \mathrm{~ms}$ of a song so the $128 \mathrm{x} 128$ pixel images equates to 2.56 seconds of audio with 128 frequency bins along the $\mathrm{Y}$ axis. A working example of this could be a 3:00 minute Pop song. The song would be converted to a spectrogram which would be 9,000 x 128 pixel image. It would be sliced up into smaller snippets. Slicing the image would create 70 - 128 x 128 snippets all labelled as Pop. Despois used 12,000 images per genre which equaled 72,000 images in the dataset. Despois used 70\% of this dataset for training, $20 \%$ validation, and $10 \%$ for testing. Once this dataset was built, the training and validation sets were used during training as input into the $\mathrm{CNN}$. The architecture of the $\mathrm{CNN}$ used by Despois is displayed in Figure 16 which consisted of four CNN layers, a fully connected layer, and a softmax function to classify the results into the genre classes specified.

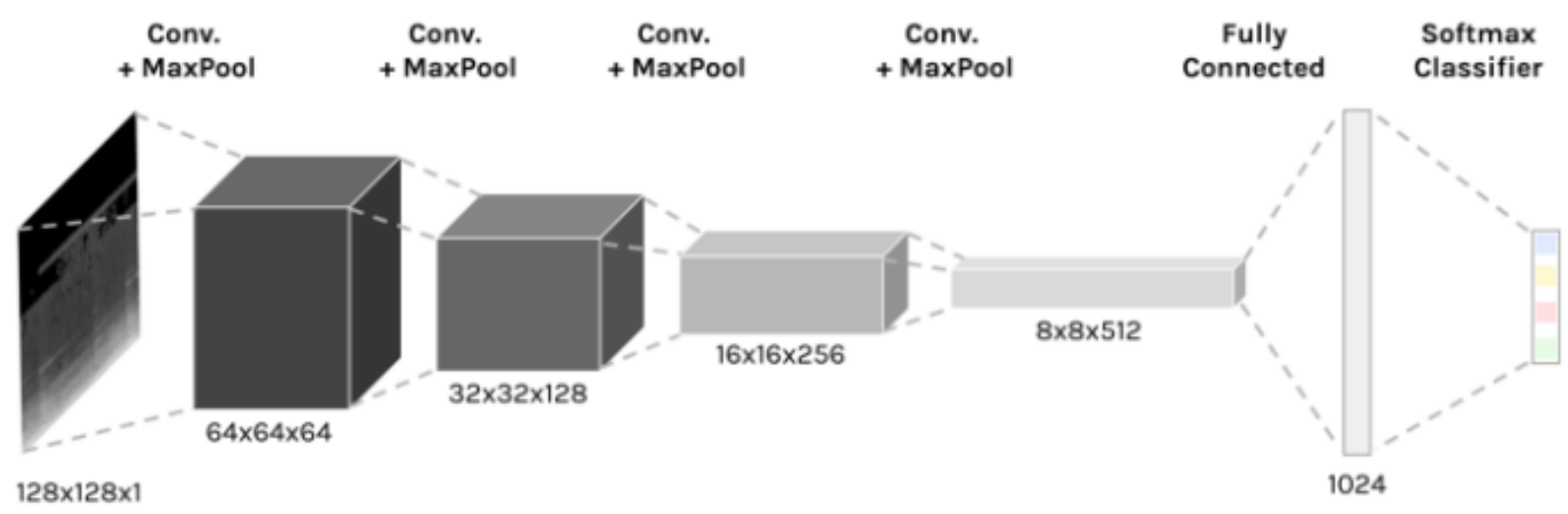

Figure 16 CNN architecture used by Despois. Source [42].

The test set accuracy of the work was not provided by Despois; however, the reported validation accuracy was $90 \%$. Some future research mentioned tweaks to the code that could be done which might vary the accuracy of the NN. One of the changes included making sure that a song's spectrogram slices were used in one of dataset segments (training, validation, or test sets) and not all three, which could potentially bring down the accuracy if the NN was being tested with the same data it was training on. 
The use of Mel scaled spectrograms has been explored in various papers [43-45]. The premise behind Mel spectrograms is supported by domain knowledge about the human auditory system and has been proven by performance gains in various tasks $[44,45]$. On the contrary, the use of Shorttime Fourier Transform (STFT), which is the equivalent of a linear spectrogram, provides finer resolutions than Mel spectrograms for frequencies greater than 2,000 Hz. This may be desirable for some tasks; however, it has not been the most favored choice in the topic of genre classification using deep learning models [44].

A direct comparison between Mel spectrograms, linear spectrograms and MFCC was done by Choi, Fazekas, and Sandler [44]. The dataset chosen for the comparison was the MagnaTagATune dataset which has a total of 188 different tags which can include genre, instruments used, and mood of the 29 second clips. For their work, they refined the 188 tags down to 50 tags by merging together very similar tags, which was also done by [40]. Something to note is that there can be more than one tag attached to a song clip in the dataset, which is different than other common datasets. This means the measure of accuracy within their work was calculated as AUC-ROC. They evaluated linear spectrograms, Mel spectrograms, and MFCC representations as input into a fully convolutional neural network (FCN). The linear and Mel spectrogram inputs were produced from the 29 second clips as $96 \times 1,366$ pixel images. They did not slice these images up before feeding it into their network as inputs. The FCN architecture used for their first experiment in comparing the three types of inputs consisted of four convolutional layers with an average-pooling at the final convolutional layer.

The results illustrated in Table 4 show that with respect to the FCN input representations, using Mel spectrograms resulted in greater accuracy compared to linear spectrograms and MFCCs [44]. 
This result aligns with the preferences of Mel spectrograms over linear spectrogram on automatic tagging [44].

Table 4 Comparison of linear spectrograms, Mel spectrograms, and MFCC by [44].

\section{Type of Input}

Linear Spectrogram (STFT)

Mel Spectrogram

MFCC

After the research showed that the Mel spectrogram was the preferred input type for classifying

\section{AUC-ROC}

\subsection{6}

0.894

0.862

music by genre [44], Panwar, Das, Roopaei et al. [43] started experimenting with Convolutional Recurrent Neural Networks (CRNNs) for genre classification. A CRNN is a neural network with both convolutional layers and recurrent layers. The benefit to this architecture is designed to allow the $\mathrm{NN}$ to understand local and temporal features from the input images, in this case, spectrogram inputs. The local features were learned through the CNN layers and the temporal and global features were learned through the RNN layers [43].

Panwar, Das, Roopaei et al. [43] used the MagnaTagATune dataset and they refined the 188 tags down to 50 tags by merging together very similar tags, similar to [40,44]. They converted the 29 second clips into Mel spectrograms using the Librosa library [29] which produced images of $96 \mathrm{x}$ 1,366. The image height represented $96 \mathrm{Mel}$ bins and the 1,366 pixel length was the total number of frames used [43]. Similar to [44], the images were not sliced up before being used as input into their network. The images went through four convolutional layers, two recurrent layers, and finally a fully connected layer to calculate the output values. The AUC-ROC value obtained in [43] was 0.893 which was comparable with the other works presented with the same type of calculation. This was also comparable to the AUC-ROC value presented in [44] as 0.894 which came from the same dataset. 
Dieleman and Schrauwen [45] did an examination into whether it was more beneficial to train a $\mathrm{NN}$ on raw audio signals or Mel spectrograms created from the raw audio [45]. The reason for their choice behind the Mel spectrogram was because most researchers do not use linear frequency spectrograms [45]. The Mel scale or a logarithmic frequency scale were preferred instead because "they reduce the resolution for higher frequencies, which matches the human perception of frequency, and reduce the size of the representation" [45]. In the years leading up to their work, feature learning had become increasingly popular with the main benefit being the researchers who built the models only needed a minimum of prior domain knowledge [45]. In other words, instead of the researchers extracting specific features they believe were important, they could create a spectrogram or some other type of mid-level representation of the audio. This still requires domain knowledge to extract this data from the dataset and the research presented in [45] explores if the NN could learn directly off the raw audio signal which would greatly reduce the need for prior knowledge about the problem.

For the two tests, Mel spectrograms versus raw audio signal, the same CNN architecture and dataset were used. The CNN was comprised of two convolutional and two fully connected layers with max pooling between layers. The dataset used was MagnaTagATune which consists of 29 second clips and had been reduced to have 50 tags, similar to $[40,44,45]$. The measured value noted in this work was AUC-ROC because the dataset used consists of multiple tags per audio clip. To compute the Mel spectrograms for input into the $\mathrm{CNN}$, a window of about 3 seconds of audio was used. For the raw audio signal, a window of about 3 seconds was also used. To compute the tag predictions for a clip within the dataset, they averaged the predictions over a consecutive window. Training the $\mathrm{CNN}$ from spectrograms gave 0.881 and training the $\mathrm{CNN}$ from the raw audio signal gave 0.849 . The Mel spectrogram approach consistently outperforms the raw audio 
signal while testing different window lengths and strides for the spectrograms and raw audio. "Although the performance level of a spectrogram-based approach was not reached, [it was] shown that the networks are able to learn useful features from raw audio" [45]. 


\section{METHODOLOGY \& IMPLEMENTATION WORK}

In this section, a CNN architecture is proposed that trains on the spectrograms of songs which have been prelabelled by musical genre. The proposed CNN extends the research done by Despois [42] by fixing inherent issues with the work, extending the work by introducing different types of spectrograms, and doing an analysis of the spectrogram results. No other comparisons were found in previous research which compares linear, Mel scaled, and logarithmic spectrograms. The CNN model established as a result of the training can subsequently categorize the musical genre of spectrograms of songs that have not been previously processed by the CNN. To achieve this, a number of steps were taken that include:

1. The gathering of a music library

2. The preprocessing of spectrograms from the music library for the $\mathrm{CNN}$

3. Extracting a dataset from the spectrograms

4. Creating a $\mathrm{CNN}$ architecture

5. Testing the trained model for accuracy using numerous measurements

6. Analysis and comparison of three types of spectrogram images used

The above steps began with the gathering of a music library consisting of raw audio files across numerous genres. Every song within the music library went through preprocessing which outputted one spectrogram per song. The spectrograms were then sliced up into multiple smaller time-slices for each song to create a larger dataset to train from. The music library, full spectrograms, and time-slices in this section are collectively called a collection. A dataset was created using the spectrogram slices within the collection and was then used as input into the NN for training and testing. A dataset does not necessarily consist of all the slices within the collection because the 
number of slices to extract from the collection was dynamically set. For the collection and dataset, if the collection was large enough, the size of the dataset could be easily changed by dynamically configuring the number of slices per genre and pulling them from the collection into the dataset currently being used.

\subsection{Collecting Music Libraries}

An initial music library was gathered and compiled for this work and is denoted in this thesis as Collection A. There were some disadvantages to this collection determined as the research progressed. The disadvantages were mainly the limited number of songs and uneven number of songs per genre which negatively affected the network. This prompted the assembly of the music within Collection B which was used for the research following the initial research results obtained with Collection A.

\subsubsection{Collection A}

Collection A's music library consisted of 1,918 songs in MP3 format which were labelled with a genre by Apple Music. Every MP3 audio file used to create the music library had a maximum length of 3:00 minutes. If the original song was longer than 3:00 then the rest of the song was discarded. The music library for this collection was $10.6 \mathrm{~GB}$ in size. The 1,918 songs were already labelled with their respective genres; however, there were about 27 genres labelled throughout the whole music library. This proved to be an insufficient number of examples per genre to train from so modifications were made to limit the number of genres to a maximum of 10 . This was done by consolidating the numerous sub-genres into 10 main genres. There were two main ways genres were combined. The first was to combine similar sub-genres so there were a core number of genres with enough data in each to train a NN. For example, Classic Rock, Rock, and General Rock were classified into one main genre labelled Rock. The other was to combine different spellings of genres 
like HipHop, Hip-Hop, and Hip Hop into one main genre labelled Hip Hop and similarly R\&B, RB Soul, and RB were combined into one main genre labelled RB Soul. The final 10 genres associated with the music library in Collection A are listed in Table 5 along with the number of songs in each genre.

Table 5 Songs per genre within Collection A's music library.

Genre

Alternative

Classical

Country

Dance

Electronic

Hip Hop

Pop

Rap

RB Soul

Rock

Total

\section{Number of Songs} 134

42

474

71

28

10

640

214

107

198

Collection A was used for any initial experiments involving different $\mathrm{CNN}$ architectures. In the very early stages of training, it became obvious that the network was being negatively affected because the number of songs per genre were not equivalent. The genre Hip Hop had a total of 10 songs while the genre Pop had 640 songs. There was no easy way to even out the number of songs per genre in Collection A's music library. Another disadvantage with Collection A was the song lengths. With each song having a maximum length of 3:00, this limited the spectrogram size produced which ultimately limited the data the $\mathrm{CNN}$ could train from. It was these disadvantages that led to the gathering of Collection B, along with the necessity to ensure the proposed CNN architecture worked similarly with other music libraries. 


\subsubsection{Collection B}

Collection B was compiled from a music library donated for the purposes of this research. One of the main advantages of this music library was the amount of songs, $50 \mathrm{~GB}$ of audio MP3 files. The number of songs per genre used to compile Collection B's music library was more than Collection A because of the increased variety of songs within the donated music library. The genres in Collection B were chosen to be the same as Collection A with one genre difference, swapping out Dance from Collection A for Blues in Collection B. The reason behind this was because there were not enough Dance songs in Collection B's music library. The number of songs within each of the 10 genres are shown in Table 6.

Table 6 Songs per genre withing Collection B's music library.

\begin{tabular}{ll} 
Genre & Number of Songs \\
\hline Alternative & 282 \\
Blues & 205 \\
Classical & 184 \\
Country & 344 \\
Electronic & 181 \\
Hip Hop & 354 \\
Pop & 266 \\
Rap & 246 \\
RB Soul & 237 \\
Rock & 811 \\
Total & 3,110 songs (17.6 GB) \\
\hline
\end{tabular}

After selecting the songs to create Collection B's music library, there were a total of 3,110 songs which were all the full length of the original song. The music library that created Collection B was 17.6 GB in size. 


\subsubsection{Differences in Collection $A$ and $B$}

One observation to note is the number of songs in Collection B was about $160 \%$ more than the songs in Collection A. There were two reasons for adding significantly more songs to the second music library. The first was to ensure that Collection A and B could be directly compared. As many variables as possible were kept constant between the two collections while directly comparing them. This included keeping the uneven number of songs per genre from Collection A and applying it to Collection B to see if the accuracy was similar. In Collection A, one genre has 640 songs while another has 10 songs. To mimic this in Collection B, at least one genre needed 640 songs and another needed a minimum of 10 songs. The number of songs pulled from the collection into the dataset for training was configurable so the number of songs per genre in each collection does not need to be identical. It is the datasets created from these collections that need to match.

Another reason Collection B had more songs than Collection A was to be able to test if having an even number of songs per genre increases the accuracy of the network. Collection A had a total of 1,918 songs in the music library. To create a similar sized dataset, Collection B needed about 200 songs per genre. 200 songs in each of the 10 genres makes 2,000 total songs which would be similar in size to Collection A. As shown in Table 6, each genre in Collection B had around 200 songs minimum. There are three genres which have significantly more than 200 songs and this was to satisfy the direct comparison between Collection A and B, as explained in the above paragraph.

\subsection{Creating Spectrogram Sets}

In this work, all data went through the same preprocessing steps prior to being used as input into the NN. This was to ensure the same preprocessing steps were applied to all training, validation, and testing data. The preprocessing was completed before splitting up the data into their respective categories. 


\subsubsection{SoX based Spectrograms}

The first step in the preprocessing of the MP3 files was to translate the MP3 into a spectrogram using SoX and then label the spectrogram with the genre from the MP3 file. There was one spectrogram produced for every MP3 file in the collection's music library. The spectrograms were added to the collection and used at a later time.

A 3:00 minute long stereo song using a sampling frequency of $44,100 \mathrm{~Hz}$ would produce $7,938,000$ samples. To reduce this to a more manageable size, the stereo channel was discarded because it contains highly redundant information for the majority of songs [42]. The SoX library was used to easily discard the right channel while retaining the left channel. Using the channel's data, a spectrogram was created utilizing SoX [31] which is a free code library that can be imported by Python. To create the spectrograms, the first 20 seconds was trimmed off while loading the MP3 file. This was done because most songs do not start immediately. A spectrogram of Pop music created from an audio file containing 12.8 seconds is shown in Figure 17 with labelled axes. This spectrogram was created only for demonstration purposes because the spectrograms used for training do not have labelled axes. A more realistic example of a spectrogram used for training is presented in Figure 18.

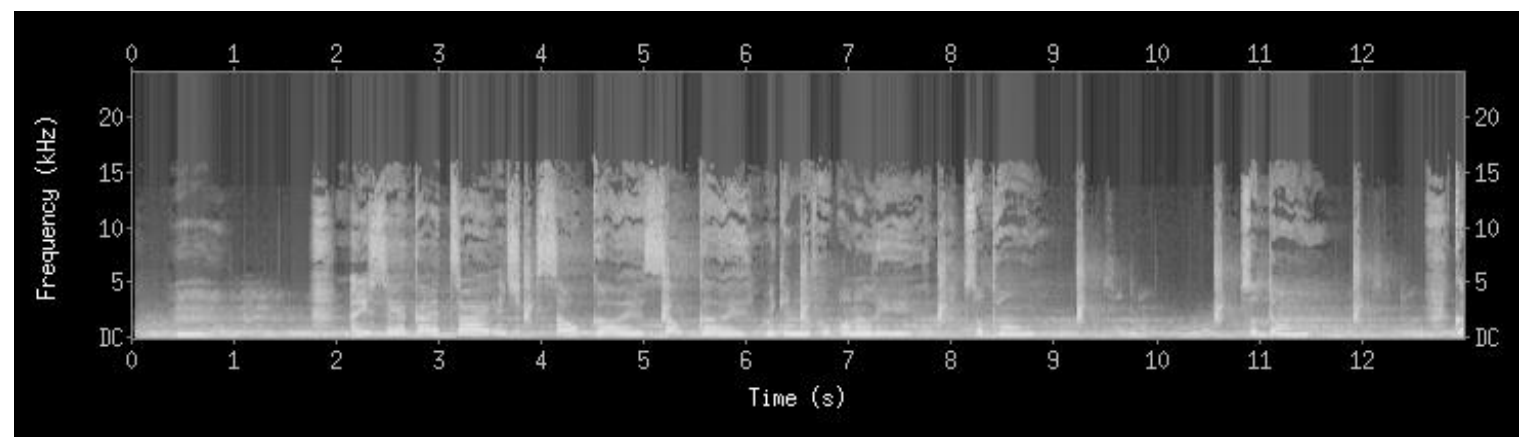

Figure 17 Spectrogram of 12.8 seconds of a Pop song created by SoX with labelled axes. 
Figure 18 Example of a full 9,000 x 128 pixel spectrogram created by SoX.

A song with a length of 3:00 minutes was transformed into a spectrogram of 9,000 x 128 pixels. While creating a spectrogram, there are numerous parameters that can be changed which include $\mathrm{X}$ axis resolution, $\mathrm{Y}$ axis resolution, $\mathrm{Z}$ axis resolution (brightness of the image), axis labels, colored or monochrome image, sampling rate, windowing function used while calculating the Discrete Fourier Transform, and several others. Along the $\mathrm{X}$ axis of the spectrograms created for this work is time; 50 pixels per second of raw audio (20ms per pixel) was chosen. The sampling rate used was $44,100 \mathrm{~Hz}$ which allows the reconstruction of frequencies up to $22,050 \mathrm{~Hz}$ which is the upper limit of the human audible range [42]. Along the $\mathrm{Y}$ axis $22,050 \mathrm{~Hz}$ in 128 pixels is about $172 \mathrm{~Hz}$ per pixel. The spectrograms were monochrome with the 'brightness' level set by the amplitude. The final configuration involved changing the digital sound processing (DSP) window function used when converting the MP3 file to a spectrogram. The DSP windowing default is the Hann window in SoX, "which has good all-round frequency-resolution and dynamic-range properties" [31]. After assessing the Hann, Bartlett, Hamming, Rectangular, and Kaiser windows on Collection A it was discovered the Hamming window provided the most accuracy for this work so the windowing function was changed to use the Hamming window function for creating the spectrograms. The Hamming window is known for better frequency resolution but lower dynamic range [31]. The description of the Hamming window aligns with other conclusions found within this research.

\subsubsection{Librosa based Spectrograms}

As described in Chapter 2, there are numerous types of spectrograms. This work focuses in on linear frequency spectrograms, Mel scaled spectrograms and logarithmic spectrograms. SoX was used to create linear frequency scaled spectrograms during the early stages of this work. SoX can 
only create linear frequency spectrograms so in order to test the proposed CNN model with different types of spectrograms, the SoX library [31] needed to be replaced with the Librosa library [29]. Librosa has options to create linear spectrograms, Mel spectrograms, as well as logarithmic spectrograms.

While replacing SoX with Librosa, the main goal was to ensure the linear spectrograms created by SoX included the same image features as the linear spectrograms from Librosa. Figure 19 compares examples of the spectrograms created by SoX and Librosa. Each of the three examples are from a different section in a song to ensure the song's spectrogram produced included the same image features from SoX to Librosa. The amplitude was depicted by pixel intensity which shows that the only difference between libraries is that they use an inverse image scale between them and thus the same features are seen in both the SoX and Librosa spectrograms. 

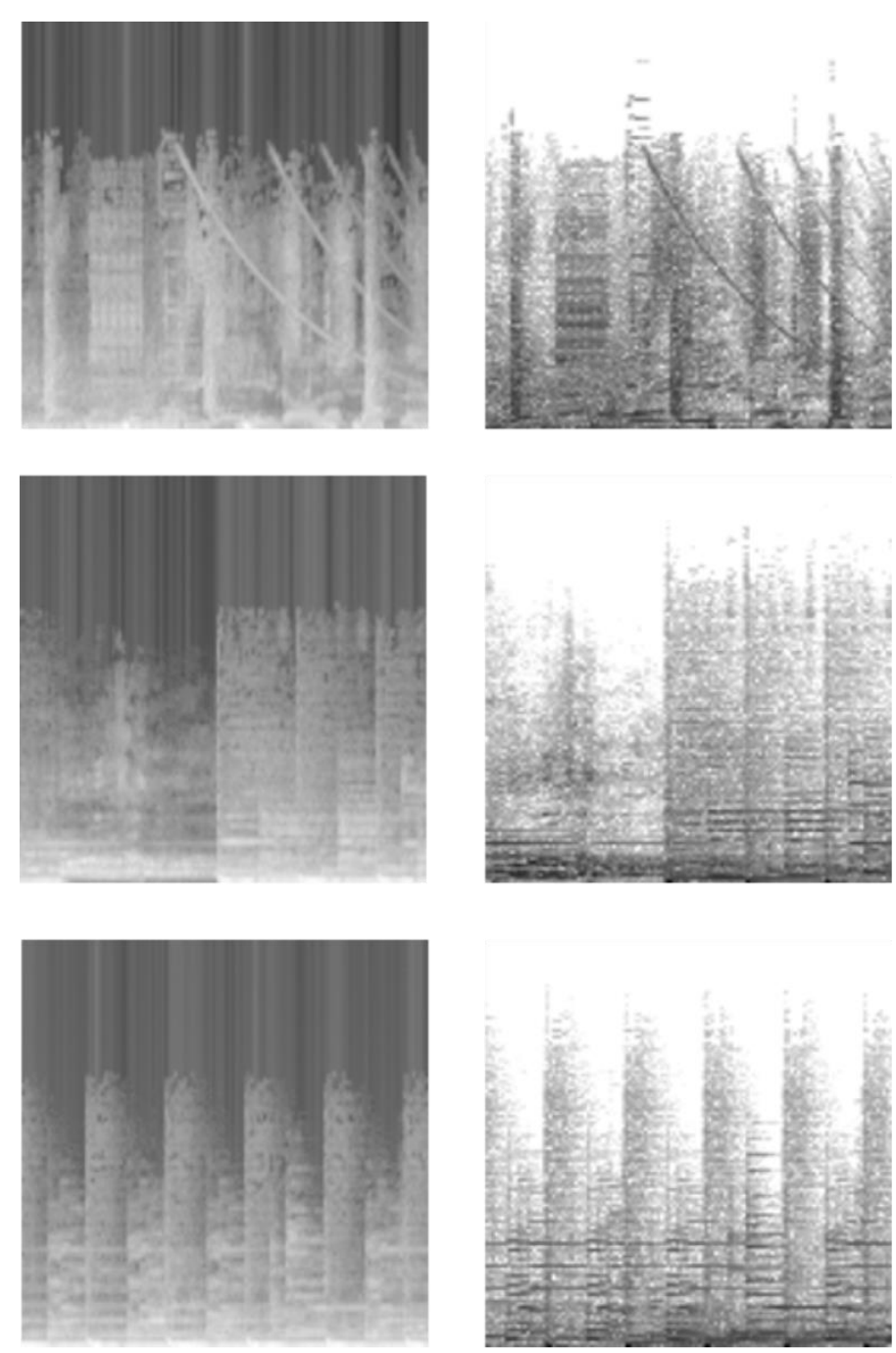

Created by SoX Library

Created by Librosa Library

Figure 19 Linear Frequency Spectrogram snippets comparing SoX versus Librosa libraries.

The Librosa spectrograms were created by first converting the audio into the Short-time Fourier transform (STFT) which "represents a signal in the time-frequency domain by computing discrete Fourier transforms (DFT) over short overlapping windows" [29]. The function in Librosa used to calculate this was `librosa.core.stft()`. After creating the STFT, it was converted from a power spectrogram to decibel units. This spectrogram data can then be plotted on a linear frequency $\mathrm{Y}$ axis or a logarithmic frequency Y axis, both of which are shown in Figure 20. 
One major difference between the images SoX and Librosa produced were the number of layers of the image. SoX created a black and white 9,000 x 128 pixel image with one layer. However, the Librosa library saved a 9,000 x 128 pixel image with three layers which corresponds to RBG layers. The reason for this was because Librosa gives the option to change the color of the amplitude of the image. The proposed CNN model is based on the input spectrogram having only one layer. Since a black and white scale was chosen, all three layers of the image saved by Librosa are the same. This means that while the images saved have three layers, only the first layer was used while training and testing the $\mathrm{NN}$ proposed.

After successfully replacing the SoX library with Librosa by comparing linear spectrograms, the same process was done to create the logarithmic spectrograms since it uses the same data from the STFT function just plotted on a logarithmic graph instead of linear. After both the linear and logarithmic spectrograms were created, the next task was to create Mel spectrograms. There was a specific function named 'librosa.feature.melspectrogram()’ in Librosa which computes the Melscaled spectrogram. There were two ways to use this function, the first was to send in the spectrogram data that has already been created, for example the output of `librosa.core.stft()`. The ‘librosa.feature.melspectrogram()` function will map the spectrogram frequency data directly from linear to Mel scale. The second way was to send in the raw audio file and it will create a Mel based spectrogram from the file [29]. Similar to the linear and logarithmic spectrograms being plotted using their respective axes, the Mel spectrogram data from the function was then plotted using a Mel frequency $\mathrm{Y}$ axis. Figure 20 shows a small section of a spectrogram created by SoX and Librosa, the equivalent of 2.56 seconds of a song. The first two images on the left are both linear spectrograms made by SoX and Librosa respectively. The third image is a Mel spectrogram and the fourth image is a logarithmic spectrogram, both created by Librosa. 
The section of spectrogram used for the comparison displayed in Figure 20 was chosen to accentuate the $\mathrm{Y}$ axis changes. In the first two images, there are curved lines swooping from the middle of the spectrogram to the bottom right of the image. In the third image, the lines are in the same region in respect to the $\mathrm{X}$ axis which is time; however, they are higher because of the Mel scaled $\mathrm{Y}$ axis. Similarly, in the fourth image the lines are in the same region in respect to the $\mathrm{X}$ axis; however, with respect to the $\mathrm{Y}$ axis, the lines are almost straight because they are plotted on a logarithmic Y axis. Please note, these curved lines were found within an example spectrogram. These images have not been manually edited to highlight the $\mathrm{Y}$ axis changes.

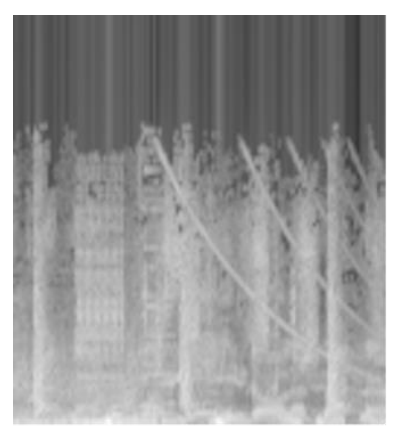

SoX - Linear

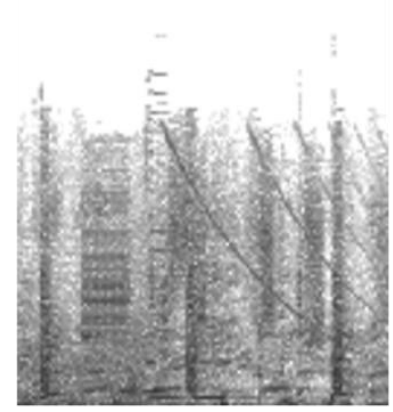

Librosa - Linear

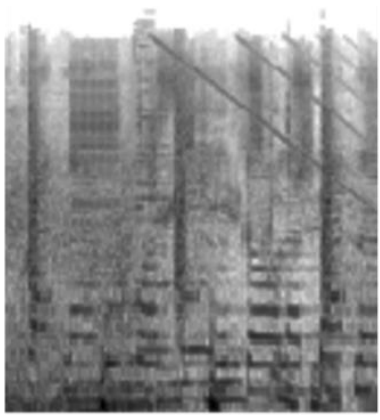

Librosa - Mel

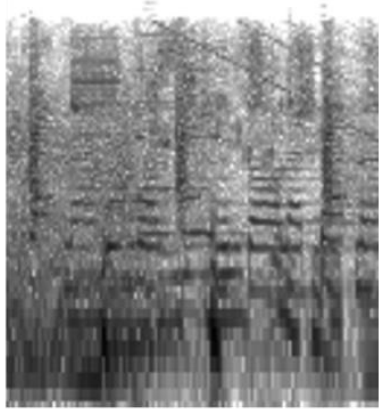

Librosa - Logarithmic

Figure 20 The same section of song converted into a spectrogram by SoX and Librosa for comparison.

Once the code was able to create correct linear, Mel, and logarithmic spectrograms it was possible to add these new spectrograms to Collection B. This was only done for Collection B because of the disadvantages with Collection A previously identified.

\subsubsection{Collection A}

Once spectrograms were created for a whole collection's music library they are added to the collection as a 'set'. Collection A consisted of one set of spectrograms which were linear spectrograms created by SoX. The number of spectrograms per genre are shown in Table 7 Spectrogram sets within Collection A. Collection A consisted of a music library containing 1,918 songs, and a linear SoX spectrogram set containing 1,918 spectrograms. 
Table 7 Spectrogram sets within Collection A.

\begin{tabular}{ll} 
Genre & Linear SoX Spectrogram Set \\
\hline Alternative & 134 \\
Blues & 42 \\
Classical & 474 \\
Country & 71 \\
Electronic & 28 \\
Hip Hop & 10 \\
Pop & 640 \\
Rap & 214 \\
RB Soul & 107 \\
Rock & 198 \\
\hline Total & 1,918 spectrograms (1.1 GB) \\
\hline
\end{tabular}

\subsubsection{Collection $B$}

Collection B's music library was used to create four spectrogram sets which included two linear spectrogram sets, a Mel scaled set, and logarithmic set. All four spectrogram sets were added to Collection B which now contains: a music library with 3,110 songs, a linear spectrogram set created by SoX with 3,110 spectrograms, a linear spectrogram set created by Librosa with 3,110 spectrograms, a Mel scaled spectrogram set created by Librosa with 3,110 spectrograms, and a logarithmic spectrogram set created by Librosa with 3,110 spectrograms. 
Table 8 Spectrogram sets within Collection B.

\begin{tabular}{lllll} 
Genre & \multicolumn{4}{c}{ Spectrogram Sets } \\
\hline Genre & Linear SoX & Linear Librosa & Mel Scaled & Logarithmic \\
\hline Alternative & 282 & 282 & 282 & 282 \\
Blues & 205 & 205 & 205 & 205 \\
Classical & 184 & 184 & 184 & 184 \\
Country & 344 & 344 & 344 & 344 \\
Electronic & 181 & 181 & 181 & 181 \\
Hip Hop & 354 & 354 & 354 & 354 \\
Pop & 266 & 266 & 266 & 266 \\
Rap & 246 & 246 & 246 & 246 \\
RB Soul & 237 & 237 & 237 & 237 \\
Rock & 811 & 811 & 811 & 811 \\
\hline Total & 3,110 & 3,110 & 3,110 & 3,110 \\
& spectrograms & spectrograms & spectrograms & spectrograms \\
& $(2.4$ GB) & $(4.6$ GB) & $(3.9$ GB) & $(4.5$ GB) \\
\hline
\end{tabular}

A name was given to each of these types of spectrogram sets to establish consistency and avoid confusion when referring back to a certain spectrogram set. They were named Collection B - Linear SoX, Collection B - Linear Librosa, Collection B - Mel, and Collection B - Logarithmic. Each spectrogram set, whether created from SoX or Librosa, consisted of the same number spectrograms as songs within the music library. The size of the spectrogram sets created by Librosa were significantly larger because the image were 128 x 128 pixels with three layers per image, whereas the images created by SoX were only 128 x 128 pixels with one layer.

\subsection{Slicing up the Spectrogram Sets}

\subsubsection{Slicing Spectrograms}

The next step in preprocessing the data for the CNN was to slice up the spectrogram sets into 128 pixel wide PNGs each representing 2.56 seconds of a given song. At the top of Figure 21 is a $9,000 \times 128$ pixel spectrogram. This is an example of a full spectrogram created from SoX. There 
is a magnified section of the spectrogram below that which correlates to 12.8 seconds of the song. The magnified section of the spectrogram is demonstrated being split up into the 2.56 second increments. There was analysis done using increments of 1.28 seconds, 2.56 seconds, and 5.12 seconds.

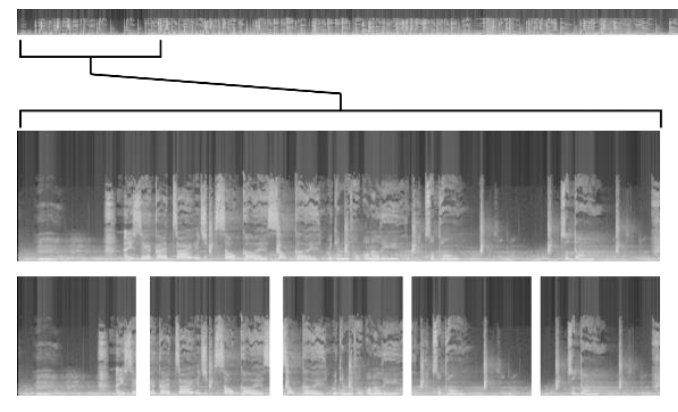

Figure 21 (Top) 9,000 x 128 spectrogram; (Middle) Zoomed in on the first 12.8 seconds of the spectrogram; (Bottom) The 12.8 seconds being sliced into 2.56 second snippets.

Figure 22 below is an enlarged portion of the bottom of Figure 21. The enlarged image shows the 12.8 second increment being sliced into $128 \times 128$ pixel images. Once this was done for an entire spectrogram, all slices produced were labelled with the song's genre. This means, for a 3:00 minute Pop song, there was $70-128$ x128 pixel images all labelled as Pop.
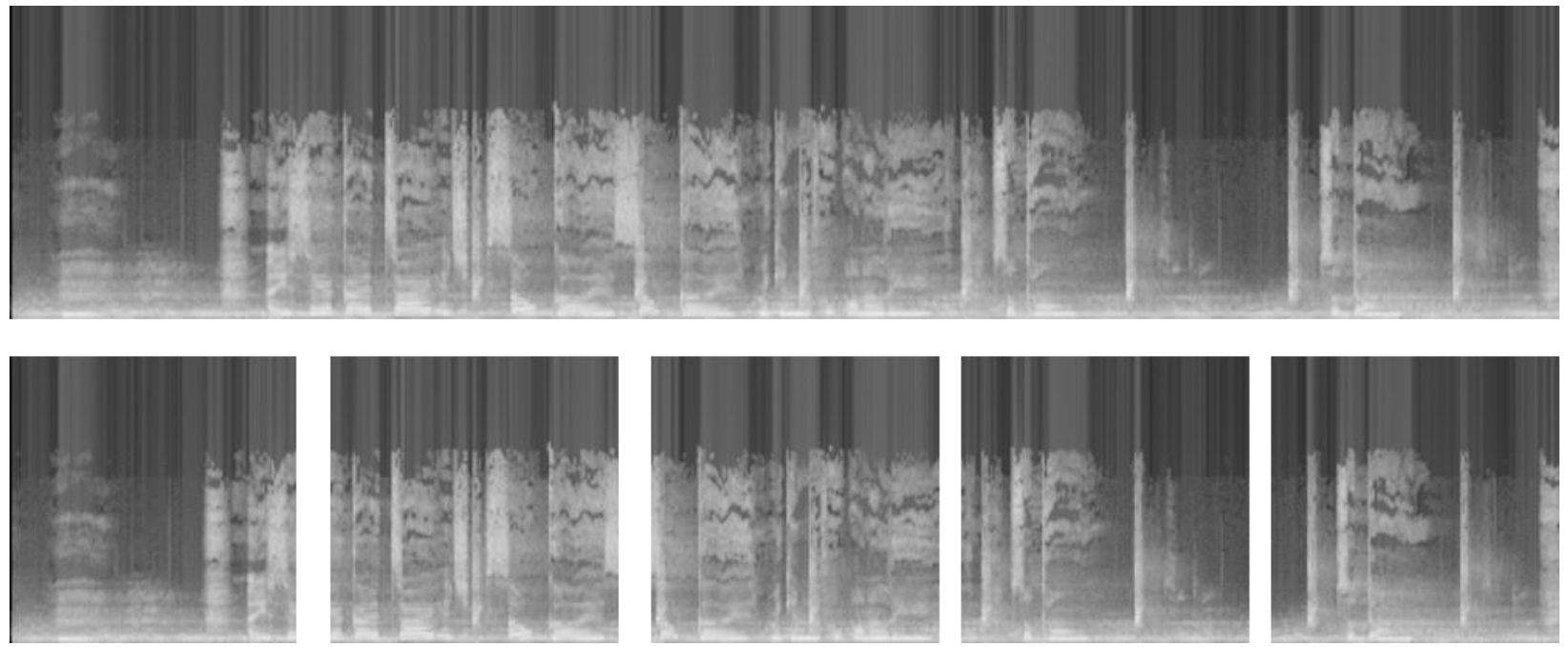

Figure 22 Enlarged portion of Figure 21; (Top) 12.8 second spectrogram of Pop music; (Bottom) divided into 2.56 second segments. 
The collection was considered complete once all the slices were added to the collection for each of the spectrogram sets within the collection. The complete collection holds the music library, spectrogram sets which consist of one spectrogram for every song within the library, and finally for every spectrogram set there is a set of slices from the spectrograms being sliced up.

\subsubsection{Contrast Filtering on Sliced Spectrograms}

Collection B was used more extensively during testing because of the shortcomings of Collection A highlighted in 3.1.1. The extensive testing done using Collection B led to filtering out spectrogram slices that were entirely white or images without significant contrast in the image. An image without significant contrast, in this context, means a contrast of less than 30 throughout the whole 128 x 128 pixel image. To calculate this contrast difference within the image, the lowest contrast pixel (blackest pixel) was subtracted from the highest contrast pixel (whitest pixel) and if that value was less than 30 , the image was not used in the collection. An example would be if the highest contrast pixel was 255 and the lowest pixel was 226 then the contrast difference for that image slice would be 29 and would not be used. For visual reference, a range of contrast from 0 to 255 is shown in Figure 23.

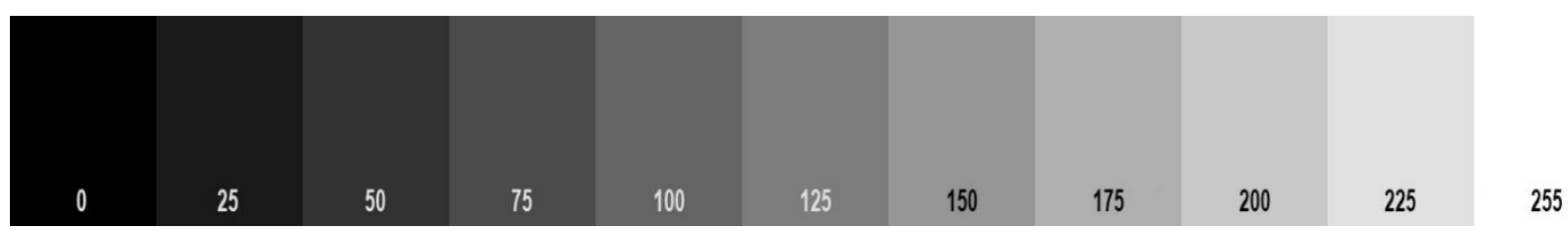

Figure 23 Greyscale contrast between full black and full white labelled with their respective RBG value.

A 2.56 second image would have a contrast of less than 30 if the song has not started, the song has ended, or there is a silent pause within the song. There was only a handful of images that were filtered out because of a silent pause within the song which can be seen in the top of Figure 24 . Most images were filtered out at the end of song when the sound quits which is shown in the bottom of Figure 24. 
Figure 24 (Top) A song that has a silent pause in the middle; (Bottom) A song finishes before the audio file is turned off.

Images without significant contrast were filtered out because there could be numerous genres with white images and it might affect the accuracy of the NN if there were lots of white images being labelled differently depending what genre they happened within. Some example images that have been filtered out of Collection B are shown in Figure 25. A border has been added around the spectrogram images for clarity as well as red circles to highlight the areas in the spectrogram which have darker pixels. The genre is labelled below each image as well as the contrast difference which is the difference between the darkest and lightest pixel in the image. The contrast difference is increasing from left to right. The left spectrogram has a contrast difference of 3 and it is hard to distinguishable where the darker pixels are located. The right most spectrogram has a contrast difference of 29 and red circles highlight the darker pixel clusters which are more noticeable.

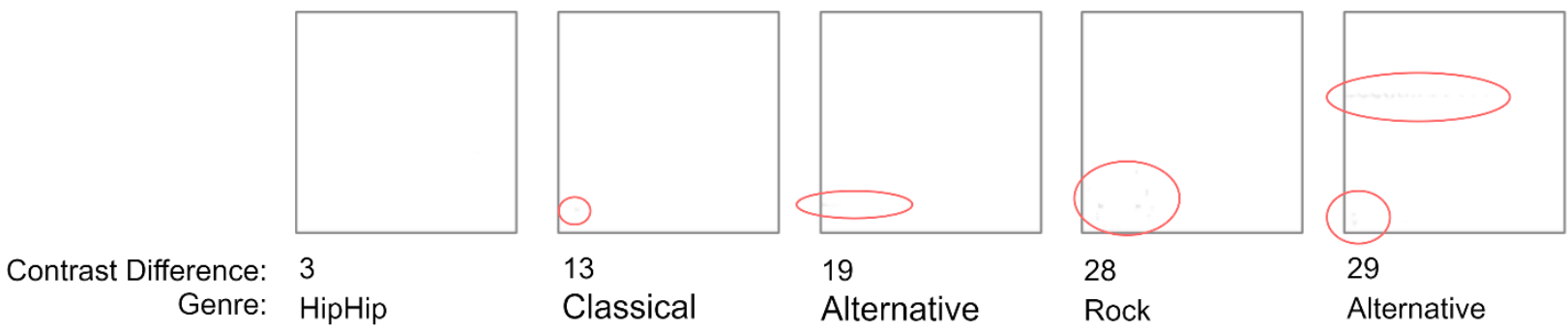

Figure 25 Spectrogram slices which were filtered out because of the lack of contrast.

As mentioned above, the filtering of white images from the sets of spectrogram slices was only applied to Collection B.

\subsubsection{Collection A}

For Collection A, the process of slicing up the spectrogram set created a total of 132,827 labelled 128 x 128 pixel slices shown by genre in Table 9. After converting the raw audio files into 
spectrograms and slicing the spectrograms into their own smaller snippets, the total size of sliced spectrograms for Collection A was 1.5 GB.

Table 9 Sets of slices within Collection A.

\begin{tabular}{ll} 
Genre & Linear SoX Sliced Spectrograms \\
\hline Alternative & 9,294 \\
Classical & 2,785 \\
Country & 32,953 \\
Dance & 4,946 \\
Electronic & 1,931 \\
Hip Hop & 700 \\
Pop & 44,420 \\
Rap & 14,852 \\
RB Soul & 7,372 \\
Rock & 13,574 \\
\hline Total & 132,827 slices (1.5 GB) \\
\hline
\end{tabular}

The final Collection A consists of:

- A music library (1,918 songs)

- A linear spectrogram set created by SoX (1,918 spectrograms)

- A set of linear spectrogram slices by SoX (132,827 spectrogram slices)

\subsubsection{Collection B}

For Collection B, the process of slicing up spectrograms needed to be applied to four spectrogram sets. All four spectrogram sets were sliced up to created their own set of slices. Each set of slices were 253,046 labelled 128 x 128 pixel slices and were added to Collection B. After creating these sets of slices, the sets originating from Librosa went through contrast filtering to filter out white images. The sets effected by the filtering of low contrast images within Collection B 
were updated. They were updated so in the future, any dataset extracted from the collection would not use these white images.

After filtering and updating these sets there was a different number of slices per set; however, the difference between sets was minimal, all within $1 \%$ of each other. The actual totals for each set are broken out per genre in Table 10.

Table 10 Sets of slices within Collection B.

\begin{tabular}{lllll} 
Genre & \multicolumn{4}{c}{ Sets of Sliced Spectrograms } \\
\hline Genre & Linear SoX & Linear Librosa & Mel Scaled & Logarithmic \\
\hline Alternative & 24,039 & 24,980 & 24,004 & 24,006 \\
Blues & 16,439 & 16,404 & 16,419 & 16,422 \\
Classical & 13,790 & 13,611 & 13,660 & 13,669 \\
Country & 22,114 & 22,091 & 22,103 & 22,104 \\
Electronic & 17,023 & 16,984 & 16,998 & 16,998 \\
Hip Hop & 29,816 & 29,734 & 29,752 & 29,755 \\
Pop & 20,787 & 20,727 & 20,749 & 20,752 \\
Rap & 21,396 & 21,376 & 21,379 & 21,379 \\
RB Soul & 16,971 & 16,963 & 16,966 & 16,966 \\
Rock & 70,671 & 69,556 & 69,649 & 69,662 \\
\hline Total & 253,046 slices & 251,426 slices & 251,679 slices & 251,713 slices \\
& $(2.8 \mathrm{~GB})$ & $(5.5 \mathrm{~GB})$ & $(4.4 \mathrm{~GB})$ & $(5.3 \mathrm{~GB})$
\end{tabular}

The images produced by Librosa were 128 x 128 pixels with three layers so the sliced up spectrogram images in Collection B - Linear, Mel, and Logarithmic took up 5.5 GB, 4.4 GB, and 5.3 GB, respectively, which is in comparison to SoX with $128 \times 128$ pixel images with one layer which totaled 2.8 GB. As mentioned before, the actual images used to train the $\mathrm{NN}$ are similar in size for all four collections since only the first layer of the images were used.

The final Collection B consists of:

- A music library (3,110 songs) 
- A linear spectrogram set created by SoX (3,110 spectrograms)

- A linear spectrogram set created by Librosa (3,110 spectrograms)

- A Mel spectrogram set created by Librosa (3,110 spectrograms)

- A logarithmic spectrogram set created by Librosa (3,110 spectrograms)

- All slices from the linear spectrogram set by SoX (253,046 spectrogram slices)

- All slices from the linear spectrogram set by Librosa (251,426 spectrogram slices)

- All slices from the Mel spectrogram set by SoX (251,679 spectrogram slices)

- All slices from the logarithmic spectrogram set by SoX (251,713 spectrogram slices)

\subsection{Extracting Datasets}

\subsubsection{Dataset Creation}

Both Collection A and B consist of a music library, spectrogram set(s) created from the raw audio, and the set(s) of spectrogram slices. After the collections were created, numerous datasets could be extracted from the collections for the purposes of training and testing the CNN. To create different datasets from the same collection, a configuration value was set which specifies the maximum number of slices per genre to extract. These configuration values can be set to a different value for every genre in the collection. Table 11 shows an example of two different datasets that can be extracted from Collection B - Linear SoX. The first column of numbers in the table are the total number of slices per genre within Collection B - Linear SoX. The second column is how many of those slices were extracted to form a dataset when the configuration value was set to 15,000 for each genre. Similarly, the third column is when the value was set to 20,000. This shows how the configuration value can easily be set to extract two separate datasets from the same collection. 
Table 11 Datasets created from Collection B while the number of slices per genre is set to a maximum of 15,000 and 20,000 respectively.

\begin{tabular}{llll} 
Genre & Slices in Collection B & $\begin{array}{l}\text { Dataset 1 } \\
* \text { Max 15,000 per genre }\end{array}$ & $\begin{array}{l}\text { Dataset 2 } \\
\text { *Max 20,000 per genre }\end{array}$ \\
\hline Alternative & 24,039 & 15,000 & 20,000 \\
Blues & 16,439 & 15,000 & 16,439 \\
Classical & 13,790 & 13,790 & 13,790 \\
Country & 22,114 & 15,000 & 20,000 \\
Electronic & 17,023 & 15,000 & 17,023 \\
Hip Hop & 29,816 & 15,000 & 20,000 \\
Pop & 20,787 & 15,000 & 20,000 \\
Rap & 21,396 & 15,000 & 20,000 \\
RB Soul & 16,971 & 15,000 & 16,971 \\
Rock & 70,671 & 15,000 & 20,000 \\
\hline Total & 253,046 slices & 148,790 slices & 184,223 slices \\
\hline
\end{tabular}

These configurable values helped balance the number of slices per genre in a dataset without having to create a new collection of data. An example of this is Dataset 1 in Table 11 where almost all of the genres had the same number of slices per genre in them which helps the NN learn each genre equally. The values can also be set to intentionally make the slices per genre disproportional as well. This technique was used to create similar datasets from Collection A and Collection B while comparing the linear SoX spectrogram slices. An example of this is in the next section.

\subsubsection{Extracting Datasets to Compare Collection A and Collection B}

The configurable values used to set the number of slices per genre in a dataset were initially implemented to help directly compare the linear SoX slices within Collection A and B. Collection A had unequal slices per genre, for example one genre had 700 slices while others had up to 44,420 slices. As such, the datasets extracted from Collection A could not have an even number of slices per genre while still having enough data to train from. Once Collection B was prepared, it was necessary to compare Collection A directly to Collection B - Linear SoX to ensure the NN 
architecture and settings were generic enough to be applied to multiple sets of data. To compare the sets of data collected, the number of slices per genre extracted from Collection B to form a dataset were deliberately unbalanced. By using the configurable values, it was easy to create this asymmetrical dataset and compare the two collections.

Table 12 shows the two datasets extracted from Collection A and Collection B, respectively. The table shows that the number of slices was unequal per genre for both datasets extracted. The two datasets were very similar in the total slices per genre, only differing in the number of slices within Collection A - Country and Collection B - Hip Hop. There were not enough Hip Hop slices within Collection B to extract 32,953 into the dataset. With the two datasets outlined in Table 12, a NN accuracy was calculated for each and compared before moving onto further testing of Collection B.

Table 12 The two datasets used to compare Collection A and Collection B directly.

\section{Dataset from Collection A $\mid$ Dataset from Collection B}

\begin{tabular}{rr|ll}
\hline Genre & Slices Extracted & Slices Extracted & Genre \\
\hline Alternative & 9,294 & 9,294 & Pop \\
Blues & 0 & 0 & Dance \\
Classical & 2,785 & 2,785 & Rap \\
Country & 32,953 & 29,816 & Hip Hop \\
Dance & 4,946 & 4,946 & Alternative \\
Electronic & 1,931 & 1,931 & RB Soul \\
Hip Hop & 700 & 700 & Country \\
Pop & 40,000 & 40,000 & Rock \\
Rap & 14,852 & 14,852 & Blues \\
RB Soul & 7,372 & 7,372 & Classical \\
Rock & 13,574 & 13,574 & Electronic \\
\hline
\end{tabular}




\subsubsection{Dataset Processing}

After creating each dataset, whether from Collection A or B, the dataset slices were split into $70 \%$ training data, $20 \%$ validation data, and $10 \%$ test data. To ensure a song's slices were not split across the training, validation and test sections, the data was only shuffled after being separated into their respective sections. This transformation is illustrated in Figure 26.

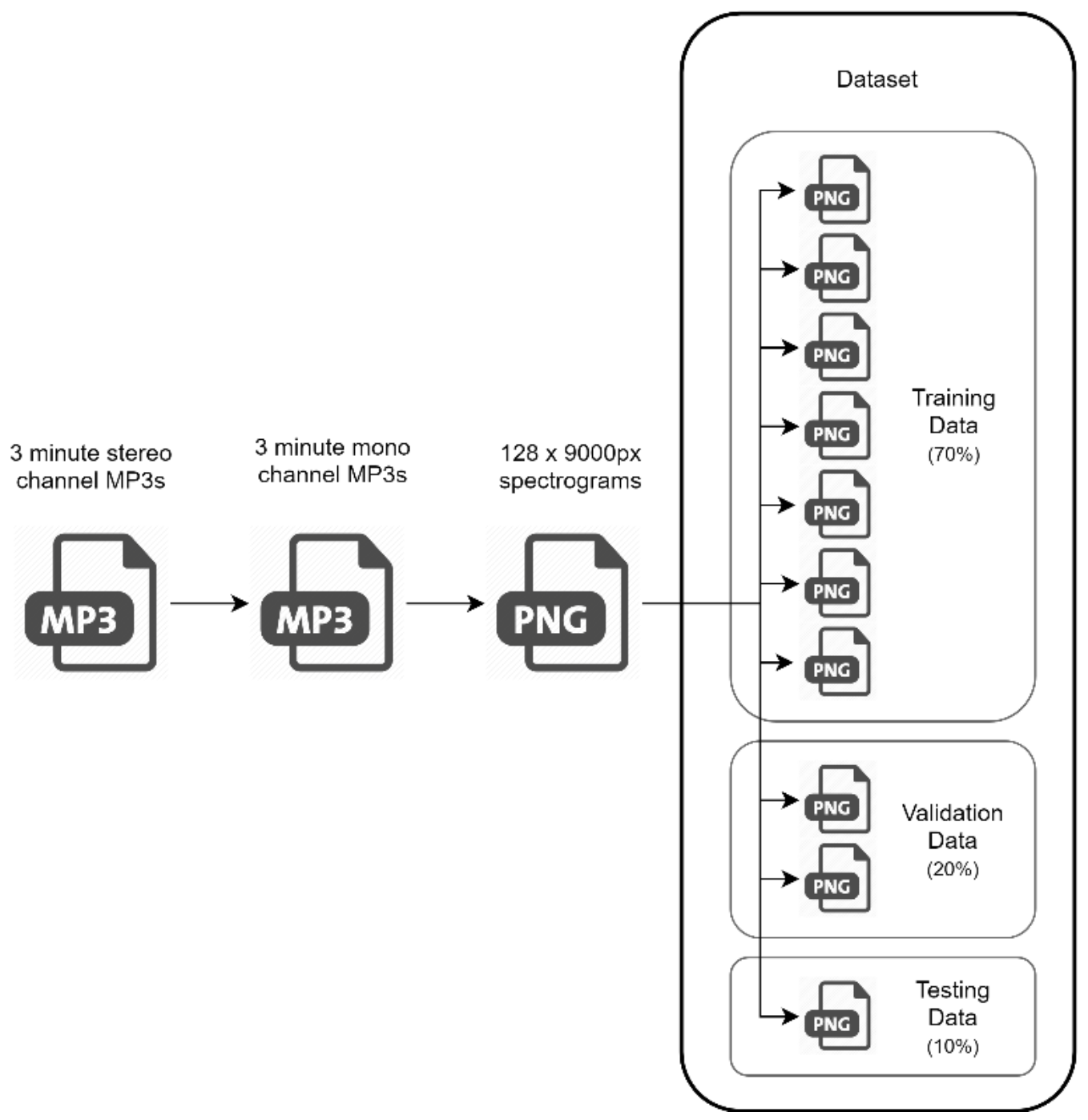

Figure 26 Preprocessing stages which took MP3 files into images in a dataset.

While using a dataset to train the $\mathrm{CNN}$ model, the training and validation data are used up. The training data is used during each iteration to train the $\mathrm{NN}$ and the validation data is used after each iteration to test whether the model is learning. This test creates the validation accuracy after each 
iteration. After the model is fully trained, the test data is used to calculate the test accuracy. This test data consists of songs the NN has not seen before.

\subsection{CNN Architecture}

A NN based off Despois [42] has been implemented in this work with several alterations. The differences include an increase in the number of music genre classes, an increase in the number of training spectrogram slices per genre used for training the NN, as well as the use of a different music library. The work extends the research in [42] by introducing different types of spectrograms and comparing results. More changes in the work include a change to the activation function, the use of different digital signal processing window functions when converting the MP3 files to spectrograms, as well as an increase in the number of CNN layers.

The neural network proposed is a deep convolutional neural network using a library built on top of TensorFlow called TFlearn [54]. TensorFlow is a very verbose open source machine learning platform whereas TFlearn is cleaner and shorter to build a fully functioning NN. The final CNN architecture is illustrated in Figure 27.

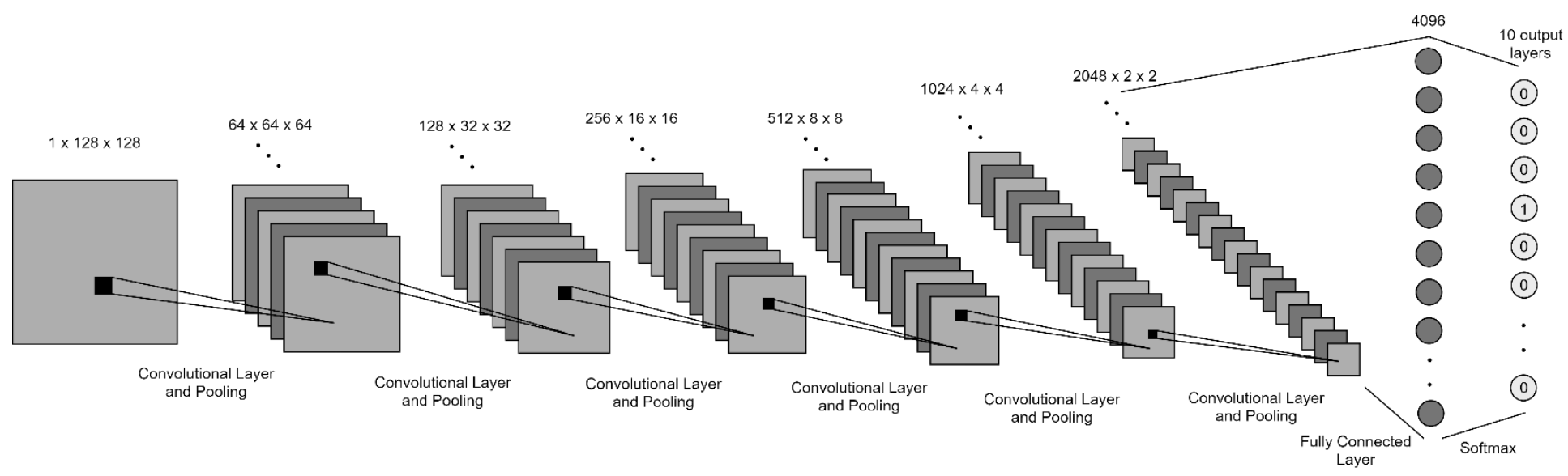

Figure 27 A diagram of the implemented CNN architecture. 
The input vector is a $1 \times 128 \times 128$ pixel spectrogram converted into a multi-dimensional vector. The first six layers are all convolutional 2D layers using the 'tflearn.layers.conv.conv_2d()' function. The extracted features in each layer are max pooled by ‘tflearn.layers.conv.max_pool_2d()` with a kernel size of $2 \times 2$ with a stride of two after each successive layer. There is a fully connected layer after the first six convolutional layers, where each output of the previous layer is fed into each input of the fully connected layer. This technique produces a 4,096 vector of numbers. Another fully connected layer with a softmax activation function is then applied to determine 10 outputs each representing a specific genre. The output from the NN is a one-hot array, which means it is an array of 0's with the chosen genre denoted as a single 1 in the output array [25]. A list of these layers and their dimensions are in Table 13.

Table 13 A list of layers in the implemented CNN architecture.

\section{Layer}

Input Spectrogram Image:

Convolution 2D + Max Pooling (2×2)

Convolution 2D + Max Pooling (2x2)

Convolution 2D + Max Pooling (2x2)

Convolution 2D + Max Pooling $(2 \times 2)$

Convolution 2D + Max Pooling (2x2)

Convolution 2D + Max Pooling $(2 \times 2)$

Fully Connected

SoftMax

\section{Output Dimension}

$1 \times 128 \times 128$

$64 \times 64 \times 64$

$128 \times 32 \times 32$

$256 \times 16 \times 16$

$512 \times 8 \times 8$

$1,024 \times 4 \times 4$

$2,048 \times 2 \times 2$

4,096

10 (One-hot array)

All the weights were initialized using Xavier initialization and the activation function being used throughout the network was a Rectified Linear Unit (ReLU). The choice to use ReLU was made after assessing the network accuracy and choosing the best performing activation function. The tests included using Exponential Linear Unit (ELU), Tanh, Leaky ReLU, and ReLU. The final layer utilizes a softmax activation function to create the output of the NN. The optimizer used was 
Adam optimizer which helps optimize the loss function. The loss function used was cross-entropy. To curb overfitting, a dropout with probability of 0.5 was implemented immediately after the fully connected layer during training. The hyperparameters used while training were a learning rate of 0.000145 , batch size of 128 and number of epochs equal to 20 . These parameters are outlined in Table 14.

Table 14 Parameters of the implemented CNN architecture.

\section{Parameter}

Input size

Output size

Learning Rate

Batch Size

Number of Epochs

Dropout

Weight Initialization

Loss Function

Optimizer

Activation function
Convolution Layers

$1^{\text {st }}$ Fully connected

$2^{\text {nd }}$ Fully connected
Value

$1 \times 128 \times 128$

10 genres

0.000145

128

20

0.5

Xavier

Categorical Cross-Entropy

Adam Optimizer

ReLU

ReLU

Softmax

\subsection{Codebase}

The music preprocessing was all done using python; from loading the MP3 song to converting it to a spectrogram to slicing the spectrogram up and saving it as smaller slices. In the same code base, python was used to create the collections, extract the datasets, and implement the CNN architecture for training and testing. The code was committed here: https://github.com/nikkipelchat/MusicGenreClassification which shows the progression of the work through pull requests. There has been work done on quality-of-life changes, preprocessing changes, and CNN architecture changes. Quality-of-life changes include being able to pause 
training while saving the weights to changes in syntax for uniformity. Preprocessing changes include the way music files are handled prior to creating the dataset for training and changes to the testing of the $\mathrm{CNN}$ by using a majority vote accuracy. $\mathrm{CNN}$ architecture changes involve changes to the number of layers and types of layers that create the model as well as changing the dimensions of the input image. There is a README.md file in GitHub to help others get up and running; however, the music libraries used in this work are not publicly available. All available commands used in the command line are listed in the README.md which include creating and slicing the spectrograms, training, resume training, testing, majority voting, and printing out a confusion matrix.

\subsection{Hardware}

Initially, training was being done on a laptop. Training times were slowly increasing as slices were added to the dataset, to the point where training was taking 26 hours. This became too time consuming because the code changes or hyperparameter changes between training could take seconds to tweak before training would begin again, all of that to note down changes a hyperparameter could make on accuracy. A computer was set up at the University of Regina so training could happen quicker and more efficiently. The code for the CNN was on GitHub so it was downloaded on the remote computer to do training only. Development happened locally and files were transferred to the remote computer via WinSCP during development. Once the code change was working, a pull request was submitted to GitHub and merged.

The GPU in the University of Regina's computer was comprised of two NVIDA GeForce GTX $1080 \mathrm{Ti}$. The GPU is important for machine learning because as a general rule, the training data is composed of simple matrix math calculations where the speed of which can be greatly enhanced if 
the computations can be carried out in parallel. There was also 32G of RAM in the computer and was utilizing a Ryzen Threadripper 1900X 8-Core Processor which runs at $2.2 \mathrm{GHz}$.

\subsection{Training and Measuring Accuracy}

Training started on a laptop which quickly became insufficient with the size of datasets, number of layers, and number of times the network needed to be trained while testing hyperparameters. After the training was moved to the remote computer located at the University of Regina, the training time was cut from 26 hours to 1 hour. After tweaks to the NN and music libraries, the training took anywhere from 1-2 hours. This timeframe depended on the size of the dataset as well as number of epochs, which is the number of times the $\mathrm{NN}$ will run through the entire dataset while training. For usability, there was a checkpoint after each epoch which saved the parameters of the partially trained network so training could be resumed at a later date.

\subsubsection{Test Accuracy}

To test the proposed model, there were two types of accuracy implemented which helped analyze and measure the final neural network in more than one area. First a normal percentage accuracy. This type of accuracy is a built in feature of TFlearn [54] to test the NN after training. The accuracy represents the amount of correct predictions in the test dataset divided by the total number of data points in the test dataset in percentage format, shown in Figure 28. For this work, this accuracy represents the percentage of correct predictions from analyzing a 2.56 second spectrogram from a song that the $\mathrm{NN}$ has never trained on or seen before.

$$
\text { Accuracy }=\frac{\text { Correct slice predictions }}{\text { Total slices in Test Dataset }} * 100
$$

Figure 28 Formula to calculate accuracy of a NN in percent. 


\subsubsection{Majority Vote Accuracy}

The second accuracy implemented was a majority vote accuracy. This accuracy was custom built by keeping track of which slices came from which songs so later there could be calculations done on each song as a whole. The song from which each slice was taken from is withheld from the NN while testing. After the NN has a prediction for each slice within the test dataset, the mode of the predictions across one whole song's slices was taken as the predicted label of the song. After each song within the test dataset has a majority vote (mode), an accuracy was calculated from how many songs were correctly labelled divided by total number of songs within the test dataset in percentage format, shown in Figure 29. The majority vote accuracy is likely to be a higher percentage than the normal test accuracy. By taking the majority vote (mode) from all song's predictions, it is limiting the odd inaccurate prediction for a slice from affecting the percentage.

$$
\begin{gathered}
\text { Song Label }=\text { Mode }(\text { Slice predictions for one song }) \\
\text { Majority Accuracy }=\frac{\text { Correct Song Labels }}{\text { Total songs in Test Dataset }} * 100
\end{gathered}
$$

Figure 29 Formula to calculate majority vote accuracy of a NN in percent.

\subsubsection{Confusion Matrix}

Finally, a confusion matrix was also implemented to help understand the mistakes between class predictions that the NN was making. The confusion matrix gives insight into the errors in predictions but also the types of errors being made. A confusion matrix is introduced in section 4.9 with more detailed explanation on how to interpret the matrix of values. 


\section{RESULTS}

In the very early stages of this research, when there had been no changes made to the architecture created by Despois [42], the music library used to create the dataset for training had 27 genres across 995 songs. It appeared the implementation of the CNN was overfit as the accuracy for the validation data was $95 \%$ while the test dataset had an accuracy of only $47 \%$. The main characteristic of an overfit $\mathrm{NN}$ model is when the difference between the validation accuracy and test accuracy is too large. Unfortunately, Despois did not report the accuracy on the test dataset but the validation accuracy reported by Despois was $90 \%$, which is similar to the initial training in this work.

Following the initial results, the music library was more than doubled to 1,918 songs, which became the music library used to create Collection A presented in 3.1.1. In addition to adding songs to the music library, modifications were made to limit the number of genres within the library from 27 down to 7 main genres (Alternative, Country, Electronic, Hip Hop/Rap, Pop, RB Soul, and Rock). Collection A has a total of 10 main genres which was used for results starting in section 4.4. The collection used for the results in sections 4.1-4.3 is denoted pre-Collection A with the only difference being the number of main genres the songs were divided into. After the addition of songs from 995 to 1,918 and reduction of genre outputs from 27 to 7 , for pre-Collection A, test accuracy was calculated to be $62 \%$.

This chapter covers results obtained from the proposed CNN model using various types of spectrograms presented in the previous chapter. The dataset extracted from the collection for training and producing results is outlined for each experiment where applicable. The dataset is described explicitly since the whole collection was not used each time. Each of the following sections detail the music preprocessing stages or $\mathrm{NN}$ architecture changes to obtain a better accuracy while utilizing Collection A and Collection B. 


\subsection{Activation Function}

The initial evaluations for the network were based on the activation function. The activation function determines whether the node should activate or not during training. This ultimately influences the routes made through the network by the training data. The activation function used in the convolutional layers was initially set to the default value, an Exponential Linear Unit (ELU). The dataset used is outlined in Table 15 and was extracted from pre-Collection A, which has 7 main genre output classifications. The spectrograms within the dataset are linear spectrograms created by SoX.

Table 15 Dataset extracted from Pre-Collection A used for testing different activation functions on the proposed CNN.

\section{Genre Dataset}

*Linear SoX Spectrogram Slices

\begin{tabular}{ll}
\hline Alternative & 9,358 \\
Country & 33,166 \\
Electronic & 6,930 \\
Pop & 40,000 \\
Rap & 15,680 \\
RB Soul & 7,070 \\
Rock & 13,876 \\
\hline Total & 126,080 slices
\end{tabular}

Changing the activation function to a Rectified Linear Unit (ReLU) resulted in an increase in accuracy of 5\%. There is no rule of thumb when it comes to activation functions, each preforms differently with the task at hand. The choice of activation function came from testing numerous functions including Tanh, ELU, Leaky ReLU and ReLU. While testing different activation functions, every other hyperparameter of the network was kept constant. The results from testing these different activation functions are shown in Table 16. The main focus of this initial experiment was to monitor the effects of the activation function on test accuracy. 
Table 16 Results from testing different activation functions on the proposed CNN.

\section{Activation Function}

Tanh

ELU

Leaky ReLU

$\operatorname{ReLU}$
Test Accuracy

$61.28 \%$

$62.45 \%$

$65.39 \%$

$67.28 \%$

After training the CNN using the ReLU activation function, the test accuracy for this work improved to $67 \%$, which was previously $62 \%$. The activation function was set to use a ReLU activation function for all future experiments including other hyperparameters, CNN architecture, and input formats.

\subsection{Length of Slices}

The next adjustment involved editing the length of the slices being used as inputs for the CNN. The datasets used for this test were extracted from pre-Collection A, which was composed of only linear spectrograms made by SoX. By default, the input length of the spectrograms after being sliced up for the $\mathrm{CNN}$ was $128 \times 128$ pixels which equated to 2.56 seconds of audio. There were two other datasets that were extracted for this test, one with smaller images and one with larger images. Smaller slices were created by using 1.28 seconds which produced an image with a size of $64 \times 128$ pixels. By increasing the slice size to 5 seconds, the images were $256 \times 128$ pixels. The datasets extracted from pre-Collection A are in Table 17 which show how many slices in each genre were used. 
Table 17 Datasets extracted from pre-Collection A used for testing varying lengths of spectrogram slices on the proposed CNN.

\begin{tabular}{l|lll} 
Genre & \multicolumn{3}{|c}{ Datasets } \\
& \multicolumn{3}{|c}{ *Linear Sox Spectrogram Slices } \\
\hline Genre & $64 \times 128$ pixels (1.28s) & $128 \times 128$ pixels (2.56s) & $256 \times 128$ pixel (5.12s) \\
\hline Alternative & 18,716 & 9,358 & 4,679 \\
Country & 40,000 & 33,166 & 16,583 \\
Electronic & 13,860 & 6,930 & 3,465 \\
Pop & 40,000 & 40,000 & 22,626 \\
Rap & 31,360 & 15,680 & 7,840 \\
RB Soul & 14,140 & 7,070 & 3,535 \\
Rock & 27,752 & 13,876 & 6,938 \\
\hline Total & 185,828 slices & 126,080 slices & 65,666 slices \\
\hline
\end{tabular}

Creating smaller slices by using 1.28 seconds of audio led to a decrease in test accuracy of $2 \%$.

It is possible 1.28 seconds is not long enough to classify a song into a genre. In contrast, increasing the slice size to 5 seconds decreased accuracy by $3 \%$. With this change, the number of labelled spectrogram samples went from 126,080 to 65,666 which might not be a large enough dataset to train with and could explain the decrease in accuracy. However, to test this hypothesis more songs would be necessary. The results from varying the length of the spectrogram slices are outlined in Table 18. The length of spectrogram slices was set to 2.56 seconds for the remainder of this work based on the conclusions obtained in this section which produced a test accuracy of $67 \%$.

Table 18 Results from varying the length of spectrogram slices on the proposed CNN.

Spectrogram Slice Length

$64 \times 128$ pixels (1.28 seconds of audio)

$128 \times 128$ pixels (2.56 seconds of audio)

$256 \times 128$ pixels (5.12 seconds of audio)

\section{Test Accuracy}

$64.92 \%$

$67.28 \%$

$63.54 \%$

\subsection{Windowing Function}

Subsequent adjustments involved changes to the DSP windowing function which was used when converting the MP3 file to a spectrogram. The dataset was unchanged from the previous section 
and is located in the middle of Table 17. The default windowing function was the Hann window which was set by SoX. After assessing the Rectangular, Kaiser, Hann, Bartlett, and Hamming windows it was discovered that the Hamming window provided the best accuracy for this work. While using the Hamming window, a test accuracy of $71 \%$ was achieved. The results from each window function test are in Table 19. The windowing function was set to use the Hamming window function for the rest of the experiments.

Table 19 Results from testing different windowing functions on the proposed CNN.

\section{Windowing Function}

Rectangular

Kaiser

Bartlett

Hann

\section{Hamming}

\section{Test Accuracy}

$63.79 \%$

$65.44 \%$

$66.38 \%$

$67.28 \%$

\subsection{Number of Genre Outputs}

With changes to the activation function and DSP window function, edits to the number of genres were altered again to understand the effects on the final accuracy. The two datasets used for this section are defined in Table 21 . The 1,918 songs were labelled with 27 genres initially and had been reduced down to the main 7 . Broadening the number of genres from 7 to 10 diminished the test accuracy by $2 \%$ which is summarized in Table 20 .

Table 20 Results from the change in the number of output classes.

\section{Number of Genres}

7 main genres (pre-Collection A)

10 main genres (Collection $A$ )

\section{Test Accuracy}

$70.71 \%$

$68.87 \%$

The decrease in accuracy was not significant given the increase in genre labels the network was now correctly classifying. The decision was made to continue classifying 10 genres (Alternative, Classical, Country, Dance, Electronic, Hip Hop, Pop, Rap, RB Soul, and Rock) instead of 7. From 
this point forward pre-Collection A which had 7 genres was not used. It was replaced with Collection A consisting of 10 genres. Both extracted datasets are shown in Table 21 for comparison. An accuracy of $69 \%$ was obtained with these changes.

Table 21 Datasets extracted from pre-Collection A and Collection A used for testing the number of output classes.

\begin{tabular}{lll} 
Genre & \multicolumn{2}{c}{ Datasets } \\
& \multicolumn{2}{c}{ *Linear SoX Spectrogram Slices } \\
\hline Genre & 7 Genres (Pre-Collection A) & 10 Genres (Collection A) \\
\hline Alternative & 9,358 & 9,294 \\
Classical & -- & 2,785 \\
Country & 33,166 & 32,953 \\
Dance & -- & 4,946 \\
Electronic & 6,930 & 1,931 \\
Hip Hop & -- & 700 \\
Pop & 40,000 & 40,000 \\
Rap & 15,680 & 14,852 \\
RB Soul & 7,070 & 7,372 \\
Rock & 13,876 & 13,574 \\
\hline Total & 126,080 slices & 128,407 slices \\
\hline
\end{tabular}

\subsection{CNN Hyperparameters and Layers}

Modification to the $\mathrm{CNN}$ settings were adjusted to maximize accuracy. The dataset used while adjusting the hyperparameters in this section is in Table 22. This was the same dataset used in the previous section. As stated earlier, a dropout with probability of 0.5 had been implemented during training. This means $50 \%$ of possible nodes were turned off at random which forces the NN to make new connections while training which aims to curb overfitting. By removing this setting, a drop of $3 \%$ in accuracy was logged, thus the setting was returned to its original state. The default optimizer was initially set to RMS Props, which is used to optimize the loss function in the network. 
Substituting this optimizer for Adam optimizer saw no change in accuracy; however, the training time for the model decreased from 75 minutes to 45 minutes so the change was preserved.

Table 22 Dataset extracted from Collection A for testing the number of hidden layers within the proposed CNN.

\section{Genre}

Alternative

Classical

Country

Dance

Electronic

Hip Hop

Pop

Rap

RB Soul

Rock

\section{Dataset}

*Linear SoX Spectrogram Slices

9,294

2,785

32,953

4,946

1,931

700

40,000

14,852

7,372

13,574

\section{Total} 128,407 slices

More changes to the $\mathrm{CNN}$ architecture included adding and removing CNN layers. Originally there were four CNN layers which produced an accuracy of $69 \%$, as seen in the previous section. By reducing the number of layers to two, an accuracy of $59 \%$ was attained. By increasing the number of layers to six the accuracy improved to $73 \%$. This rise in test accuracy prompted another test with a total of eight hidden layers to see if accuracy would increase further; however, the accuracy diminished significantly to $31 \%$. These results are summarized in Table 23 . A total of six hidden layers was used for the rest of the research results. 
Table 23 Results from changing the number of hidden layers within the proposed CNN.

Hidden Layers within the Network

2 hidden layers

4 hidden layers

6 hidden layers

8 hidden layers
Test Accuracy

$58.63 \%$

$68.87 \%$

72.71\%

$31.19 \%$

With each extra layer, there are more weights and biases for the network to adjust [6]. This allows functions with higher complexity to be expressed in the NN model. An example of this is in Figure 30, which shows three scenarios of network models sorting the light and dark dots into their respective light and dark sections. The left most image shows a model consisting of one hidden layer with 3 neurons. This example demonstrates that it is not a deep enough network to model the data accurately because there are dark dots not encompassed by the dark section which represents data points that would be incorrectly labelled. The right most image represents a model composed of a hidden layer with 20 neurons which can express a more complex dataset but can become overfit quickly [6]. This model correctly shows that every dark dot is encompassed by the dark section. However, once new data is introduced, the dark section might not encompass the newly introduced dark dots. This scenario represents a network that is overfit. The middle image with 6 neurons in a hidden layer might be the best architecture for the example at hand because the dark section encompasses most of the dark dots and it is likely that the majority of newly introduced dots will fall within the correct sections.

Figure 30 shows a smaller sized network is appropriate for simpler data while a larger, deeper network is more suitable for complex data. The proposed CNN model benefited from more layers which could mean four layers was not deep enough to accurately depict the complexities of the music data. To curb overfit while adding more layers to the network, dropout of $50 \%$ was still being used. 

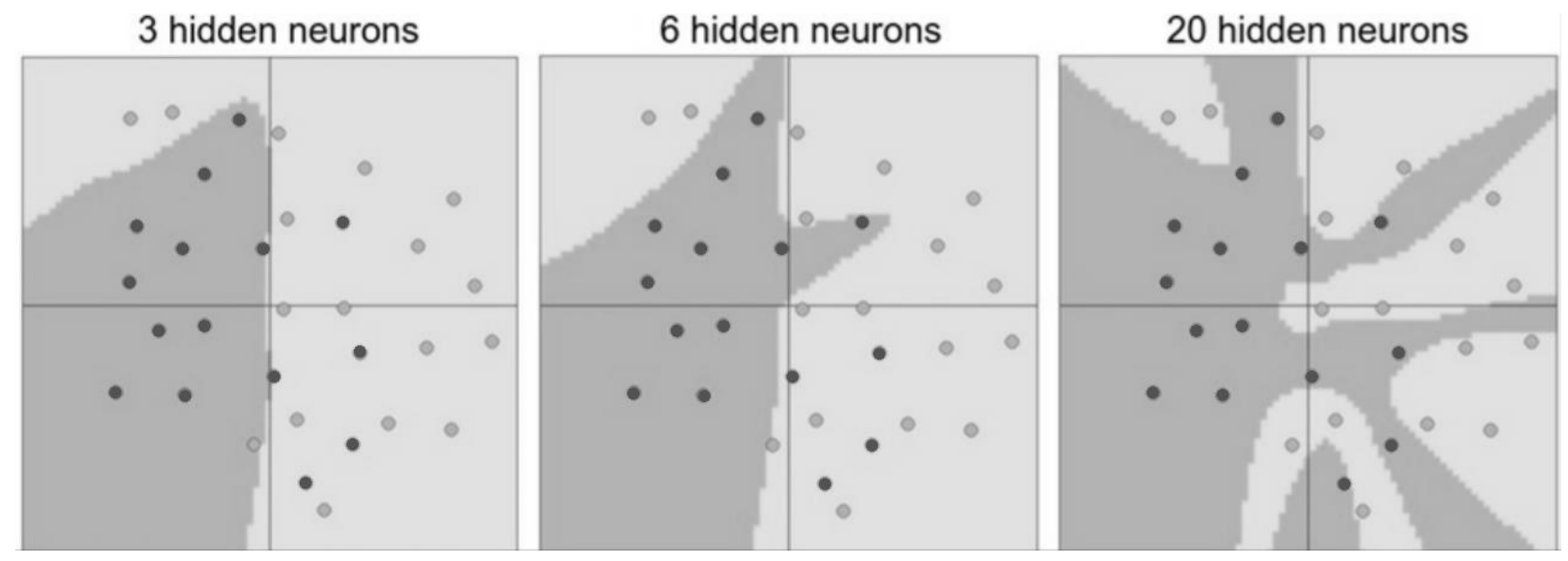

Figure 30 A depiction of how the number of neurons and hidden layers in a network can affect the final model complexity. Source [6].

\subsection{Comparison of Collection A and B}

To confirm the proposed CNN architecture and hyperparameters work using multiple music libraries, Collection B was assembled. There were also multiple disadvantages with Collection A as highlighted in section 3.1.1. These disadvantages prompted Collection B to consist of significantly more songs in the music library to ensure at least two things:

1. There must be a minimum number of songs per genre so a dataset with an even number of slices per genre can be extracted

2. A minimum number of songs per genre so a dataset that is identical to Collection A's uneven slices per genre can be extracted to compare the two collections.

The datasets extracted from Collection A and Collection B which are used to compare the collections directly against each other are outlined in Table 24 . These two extracted datasets were initially described in section 3.4.2. The number of songs per genre in both datasets are almost identical excluding one genre which differs by 3,137 slices. Having both datasets as close to identical as possible helped keep as many variables constant while comparing the two collections. 
The type of spectrogram used from each collection was the linear spectrogram created from SoX. Within each dataset the total number of slices was similar, around 127,000 , but one thing to note is the number of slices per genre was uneven. This was necessary for only the comparison, since every dataset extracted from Collection A will not have an even number of slices per genre. This was the main disadvantage to Collection A.

Table 24 The two extracted datasets used to compare Collection A and Collection B directly.

\begin{tabular}{rr||ll}
\multicolumn{2}{c||}{$\begin{array}{r}\text { Dataset from Collection A } \\
\text { *Linear SoX Spectrogram }\end{array}$} & \multicolumn{2}{l}{ Dataset from Collection B } \\
& Slinear SoX Spectrogram & \\
\hline Genre & Slices Extracted & Slices Extracted & Genre \\
\hline Alternative & 9,294 & 9,294 & Pop \\
Blues & 0 & 0 & Dance \\
Classical & 2,785 & 2,785 & Rap \\
Country & 32,953 & 29,816 & Hip Hop \\
Dance & 4,946 & 4,946 & Alternative \\
Electronic & 1,931 & 1,931 & RB Soul \\
Hip Hop & 700 & 700 & Country \\
Pop & 40,000 & 40,000 & Rock \\
Rap & 14,852 & 14,852 & Blues \\
RB Soul & 7,372 & 7,372 & Classical \\
Rock & 13,574 & 13,574 & Electronic \\
\hline
\end{tabular}

The datasets extracted from Collection A and B were then used to train and test the proposed CNN. While testing Collection A's dataset, an accuracy of $73 \%$ was obtained. This is compared with Collection B's dataset at $80 \%$ accuracy. These accuracies are shown in Table 25. This assessment was focused on the effects of different song libraries so other variables such as the NN hyperparameters were unchanged. As mentioned before, the datasets used were created as equal as possible to eliminate any differences other than the actual songs within the collections. 
Table 25 Results from comparing Collection A with Collection B using the proposed CNN.

\section{Dataset}

Dataset extracted from Collection A

Dataset extracted from Collection B

\section{Test Accuracy}

\section{$72.71 \%$}

$\mathbf{7 9 . 8 1 \%}$

It was apparent after comparing Collection A with Collection B that the proposed CNN architecture and hyperparameters work on different music libraries without having to change anything other than the raw audio songs. The variance in accuracy between the collections may be a result of the different number of slices within a genre across both datasets as well as the variance in songs within each collection. For example, each dataset had a genre with 700 slices, a genre with 14,852 slices, and another genre with 40,000 slices which helped create similar datasets for comparison purposes. However, the actual genres were different between the datasets which could potentially affect accuracy. The genre with 700 slices in the dataset extracted from Collection A was Hip Hop, but for the dataset extracted from Collection B it was Country. Similarly, for the genre having 14,852 slices, one was Rap and one was Blues. For 40,000 slices the two genres were Pop and Rock. To test whether these differences in genre could affect test accuracy, more songs would be required in Collection A since the dataset extracted was using the majority of slices available within the Collection.

This test proves Collection A and B both provide accuracies within 7\% despite their inherent differences under the same conditions and hyperparameters. This result indicates further testing can be done with Collection B given its advantages over the limitations of Collection A as described previously.

\subsection{Evenly Distributed Slices per Genre}

Given the results of directly comparing Collection A with Collection B, further optimizations were made to the datasets extracted from Collection B. The main reason to switch to Collection B 
was the ability to even out the distribution of slices for each genre within the datasets extracted. The Even Dataset in Table 26 shows that most genres have 15,000 slices in them. This ensures the model created from the $\mathrm{NN}$ has an equal representation of each genre and enough data to fully represent the genre. Classical was the genre with the minimum number of slices within Collection B. It was the genre that limited the slices to 15,000 in the Even Dataset. If 20,000 slices had been chosen, 4 of 10 of the genres would have insufficient slices to compose an evenly distributed dataset. Using 15,000 allowed for the largest dataset while still maintaining an even distribution, totalling 148,790 slices. Prior to this step in the research all datasets had been similar to the Uneven Dataset displayed in Table 26. In the Uneven Dataset, Country has insufficient data for the NN to learn the intricacies of that genre, meanwhile there was an over representation of songs classified as Rock.

Table 26 The datasets extracted out of Collection B used for comparing an unevenly distributed dataset to an evenly distributed dataset.

\begin{tabular}{llll} 
Genre & $\begin{array}{l}\text { Total Linear SoX } \\
\text { Slices in Collection B }\end{array}$ & $\begin{array}{l}\text { Uneven Dataset } \\
\text { *Mimicking Collection A's } \\
\text { uneven slices per genre }\end{array}$ & $\begin{array}{l}\text { Even Dataset } \\
\text { *Max 15,000 per genre }\end{array}$ \\
\hline Alternative & 24,039 & 4,946 & 15,000 \\
Blues & 16,439 & 14,852 & 15,000 \\
Classical & 13,790 & 7,372 & 13,790 \\
Country & 22,114 & 700 & 15,000 \\
Electronic & 17,023 & 13,574 & 15,000 \\
Hip Hop & 29,816 & 29,816 & 15,000 \\
Pop & 20,787 & 9,294 & 15,000 \\
Rap & 21,396 & 2,785 & 15,000 \\
RB Soul & 16,971 & 1,931 & 15,000 \\
Rock & 70,671 & 40,000 & 15,000 \\
\hline Total & 253,046 slices & 125,206 slices & 148,790 slices \\
\hline
\end{tabular}

The results from training the NN using the Uneven Dataset and the Even Dataset are in Table

27. While the data was unevenly distributed an accuracy of $80 \%$ was attained. After evenly 
distributing the music between the output classes of the $\mathrm{NN}$ an accuracy of $86 \%$ was reached. It was clear the model created by the NN could characterize each output class more precisely when each genre was equally represented in the dataset. The model benefits from having an evenly distributed dataset.

Table 27 Results from comparing an unevenly distributed dataset to an evenly distributed dataset, both extracted from Collection $B$.

\section{Datasets extracted from Collection B}

Uneven Dataset

Even Dataset

\section{Test Accuracy}

$79.81 \%$

$\mathbf{8 5 . 7 8 \%}$

\subsection{Linear, Mel, and Logarithmic Spectrogram Input}

The results presented to this point were acquired using linear spectrograms created by SoX. To further progress the research contained in this thesis, Collection B was expanded to contain different types of spectrograms created by Librosa. This preprocessing was covered in section 3.2 .4 and 3.3.4 which discussed the creation of linear, Mel, and logarithmic spectrograms for Collection B using the Librosa library.

While switching to the new library to create the spectrograms, comparisons between the linear SoX spectrograms and the linear Librosa spectrograms were made to ensure they produced similar test accuracies when used as input into the network. Once the linear spectrograms were created for Collection B using Librosa, a comparison of the linear SoX and linear Librosa spectrograms was done. Figure 31 shows the differences between the spectrograms produced from the opposing libraries. The differences are mainly the inverse relationship of brightness of the image but the informational content appeared to be the same. 


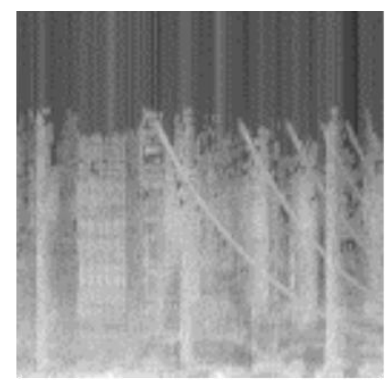

Created by SoX Library

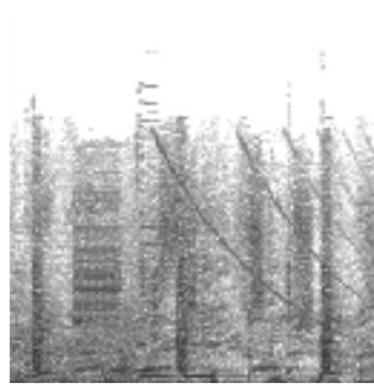

Created by Librosa Library

Figure 31 A linear spectrogram slice created by SoX and the same one created by Librosa.

The dataset used for SoX is the Even Dataset from Table 26 and the dataset for Librosa is presented in Table 29 located in section 4.8.1. There was a total of 148,790 slices in the SoX dataset and 148,611 slices in the Librosa dataset. The accuracies in Table 28 show the linear spectrograms from SoX and Librosa produce very similar results. This demonstrates that the overall greyscale information contained within the image, despite the inverse nature, does not significantly affect the training result.

Table 28 Results from comparing linear spectrograms from SoX and Librosa.

\section{Linear Datasets extracted from Collection B}

SoX

\section{Librosa}

After ensuring the linear spectrograms created from Librosa were comparable to the linear spectrograms from SoX, experiments went forward using only the Librosa spectrograms. While training the NN using linear, Mel and logarithmic spectrograms within Collection B, every variable was kept constant across the datasets when possible. This includes maintaining all hyperparameters of the network, using the same number of spectrogram slices while training and testing, and using the same songs while comparing the NN accuracies across different spectrogram datasets. 


\subsubsection{Linear Spectrograms}

The main goal while testing the linear, Mel, and logarithmic spectrograms was to produce stable results which could be compared against one another. The dataset used for testing linear Librosa spectrograms is shown in Table 29. Classical was the only genre without a total of 15,000 slices which arises because of the number of classical songs within Collection B was limited.

Table 29 The dataset extracted from the linear Librosa spectrogram slices.

\begin{tabular}{l|ll} 
Genre & \multicolumn{2}{c}{ Linear Librosa } \\
\hline Genre & Total Slices in Collection B & $\begin{array}{l}\text { Linear Librosa Dataset } \\
* \text { *ax } 15,000 \text { per genre }\end{array}$ \\
\hline Alternative & 24,980 & 15,000 \\
Blues & 16,404 & 15,000 \\
Classical & 13,611 & 13,611 \\
Country & 22,091 & 15,000 \\
Electronic & 16,984 & 15,000 \\
Hip Hop & 29,734 & 15,000 \\
Pop & 20,727 & 15,000 \\
Rap & 21,376 & 15,000 \\
RB Soul & 16,963 & 15,000 \\
Rock & 69,556 & 15,000 \\
\hline Total & 251,426 slices & 148,611 slices \\
\hline
\end{tabular}

Training the NN ten times and taking an average of the accuracy was crucial thoroughly testing the linear spectrograms created from Librosa. Taking an average accuracy over several training sessions helps create a stable result since each time the NN is trained the test accuracy can vary. The accuracy can vary because the network weights and biases are set to random values to begin training from which affects the outcome slightly. Shown in Table 30 are the results over ten training sessions. The average accuracy for linear Librosa spectrograms was $86.13 \%$. The average majority vote accuracy was $90.99 \%$. 
Table 30 Average accuracy and majority vote accuracy obtained used linear Librosa spectrograms.

\begin{tabular}{|c|c|c|}
\hline & Test Accuracy & Majority Vote Accuracy \\
\hline & $86.89 \%$ & $90.98 \%$ \\
\hline & $86.28 \%$ & $91.67 \%$ \\
\hline & $86.55 \%$ & $90.14 \%$ \\
\hline & $85.79 \%$ & $90.23 \%$ \\
\hline & $86.29 \%$ & $91.76 \%$ \\
\hline & $86.60 \%$ & $90.80 \%$ \\
\hline & $85.56 \%$ & $90.80 \%$ \\
\hline & $84.87 \%$ & $90.59 \%$ \\
\hline & $86.93 \%$ & $91.87 \%$ \\
\hline & $85.57 \%$ & $91.01 \%$ \\
\hline Average & $86.13 \%$ & $90.99 \%$ \\
\hline
\end{tabular}

With each training session, TFlearn can output the validation accuracy and validation loss after each epoch of training. This output can be used for several reasons but mainly helps to understand if the network is learning and to understand when to stop training the model to prevent it from becoming overfit. For each of the ten training sessions displayed in Table 30, the average training accuracy and average validation accuracy was plotted in green presented in Figure 32. The average training accuracy represents the accuracy of the network when tested on the training dataset. This is not the accuracy to monitor since the network is training from the same dataset as this accuracy is being calculated from. The validation accuracy is calculated from testing the current model after each epoch on never before seen data, which comes from the validation dataset. This accuracy should rise with training accuracy as seen in Figure 32; however, to ensure the model is learning appropriately from the data another important calculation to observe is validation loss. The validation accuracy and validation loss are used by the $\mathrm{NN}$ after each epoch to help confirm the model is progressing in the correct direction to reach the global minima while tweaking the weights and biases. Figure 32 shows the accuracy as a decimal percent on the $\mathrm{Y}$ axis and the epochs on the 
$\mathrm{X}$ axis. The final validation accuracy was $86.4 \%$ which was similar to the final test accuracy in

Table 30. This is a sign the NN is not overfit.

Average Training vs Validation Accuracy for Linear Spectrograms

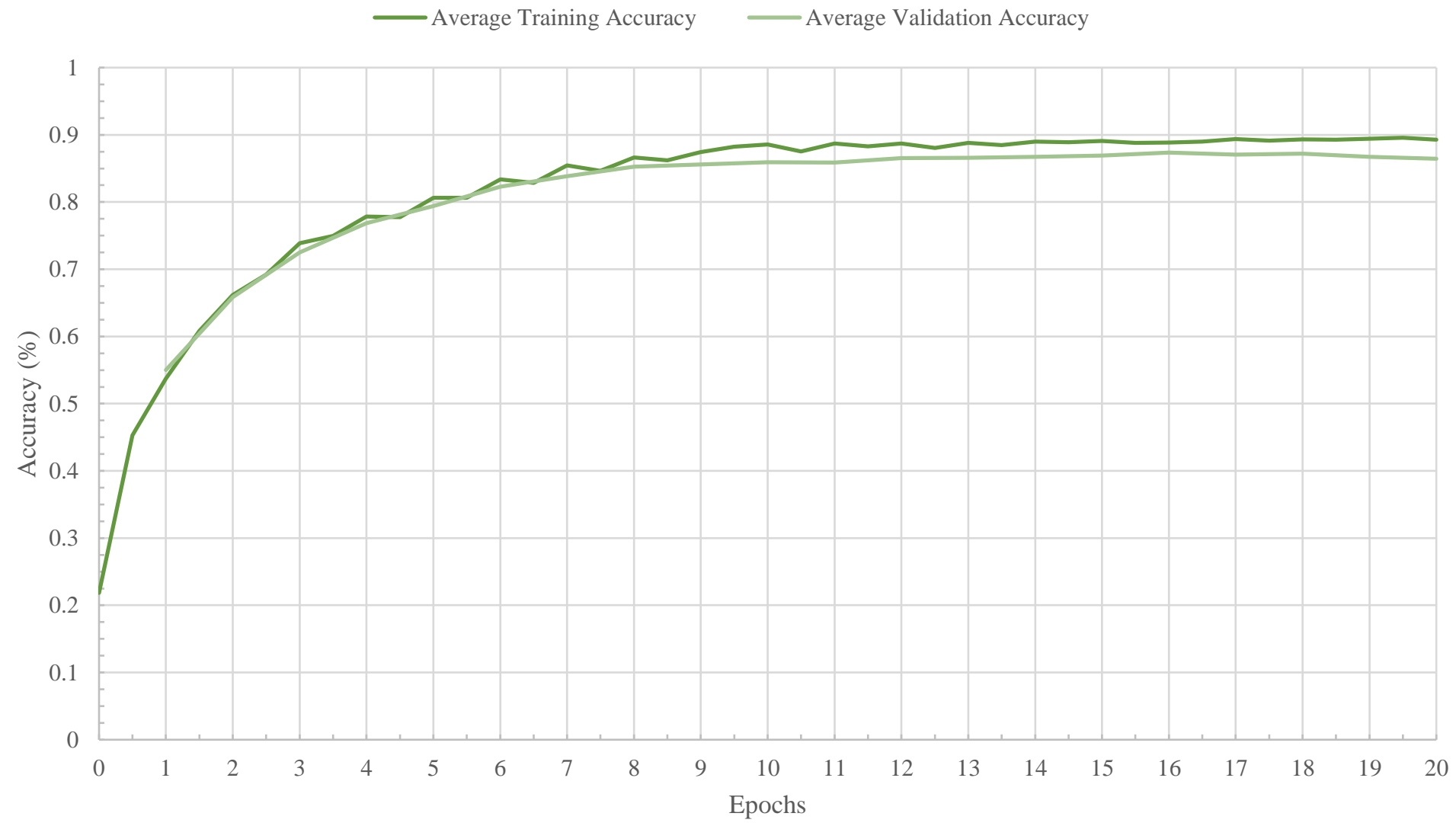

Figure 32 Average training and average validation accuracy per epoch for linear Librosa spectrogram slices.

Validation loss is the summation of errors after each epoch while using the validation dataset. Similar to the graph above, there is two loss values to plot and compare with each other. The training loss is the same calculation as validation loss except done with the training dataset. The summation of errors can be calculated in different ways, for this thesis Adam optimizer was used to optimize the loss function. Validation loss is a good indication of how the model performs on unseen data. The average training and validation loss from the ten training sessions presented in 
Table 30 were plotted in blue in Figure 33. The raw validation loss number is on the Y axis with each epoch on the $\mathrm{X}$ axis.

\section{Average Training vs Validation Loss for Linear Spectrograms}

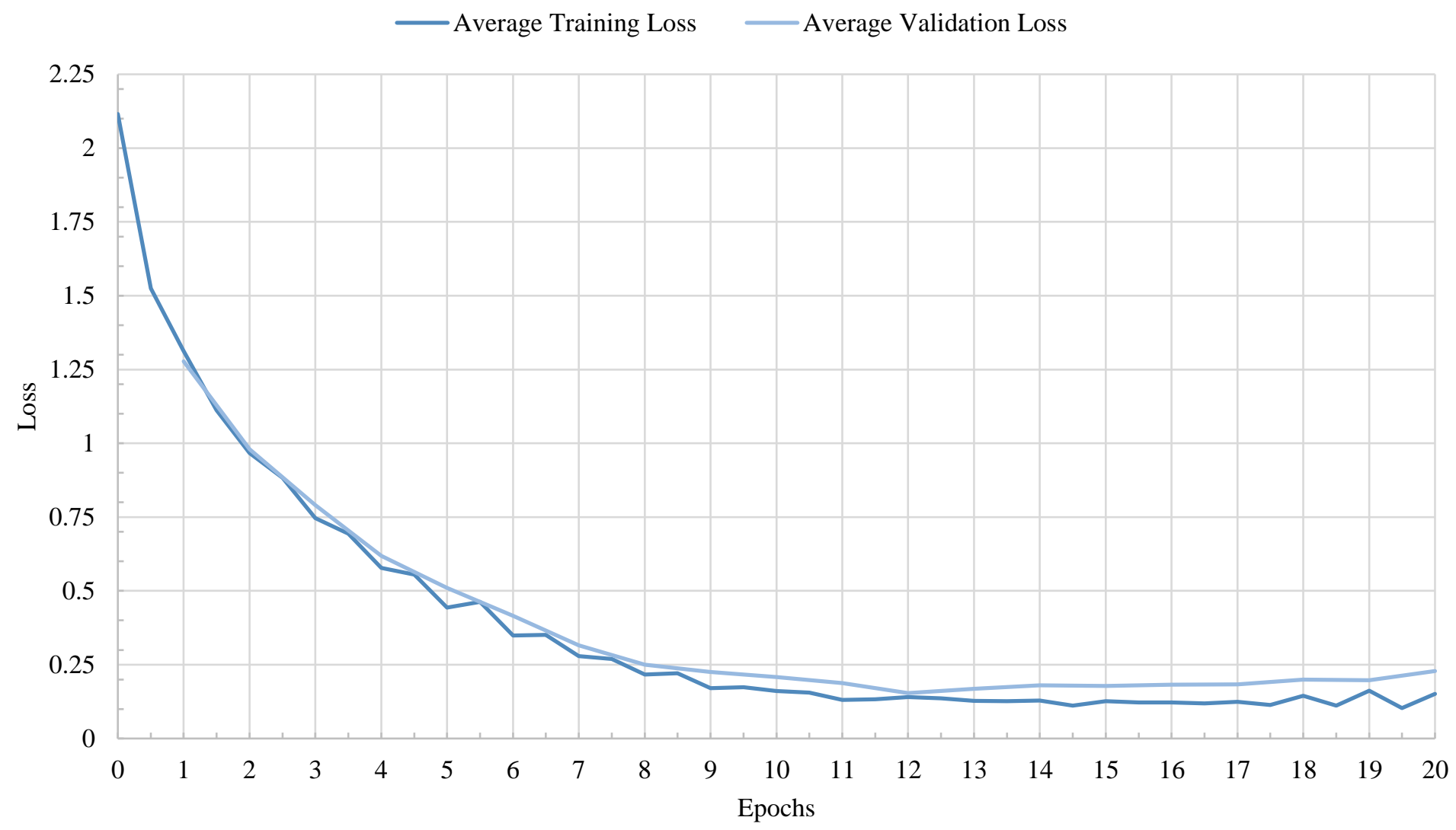

Figure 33 Validation loss per epoch for linear Librosa spectrogram slices.

\subsubsection{Mel Spectrograms}

Next was to test the Mel scaled Librosa spectrograms while keeping other factors the same. The dataset used for testing Mel Librosa spectrograms is shown in Table 31. Similar to the linear dataset, classical was the only genre without a total of 15,000 slices which was a limitation of Collection B. 
Table 31 The dataset extracted from the Mel scaled Librosa spectrogram slices.

\begin{tabular}{lll} 
Genre & \multicolumn{2}{c}{ Mel Scaled Librosa } \\
\hline Genre & Total Slices in Collection B & $\begin{array}{l}\text { Mel Librosa Dataset } \\
* \text { Max } 15,000 \text { per genre }\end{array}$ \\
\hline Alternative & 24,004 & 15,000 \\
Blues & 16,419 & 15,000 \\
Classical & 13,660 & 13,660 \\
Country & 22,103 & 15,000 \\
Electronic & 16,998 & 15,000 \\
Hip Hop & 29,752 & 15,000 \\
Pop & 20,749 & 15,000 \\
Rap & 21,379 & 15,000 \\
RB Soul & 16,966 & 15,000 \\
Rock & 69,649 & 15,000 \\
\hline Total & 251,679 slices & 148,660 slices \\
\hline
\end{tabular}

Table 32 shows the final accuracy and the majority vote accuracy of the network after ten training sessions with the average provided at the bottom of the table. The average accuracy for Mel scaled Librosa spectrograms was $86.50 \%$. The average majority vote accuracy was $91.27 \%$.

Table 32 Average accuracy and majority vote accuracy obtained used Mel scaled Librosa spectrograms.

\begin{tabular}{|c|c|c|}
\hline & Test Accuracy & Majority Vote Accuracy \\
\hline & $87.69 \%$ & $92.15 \%$ \\
\hline & $87.53 \%$ & $91.76 \%$ \\
\hline & $86.14 \%$ & $92.21 \%$ \\
\hline & $85.91 \%$ & $90.52 \%$ \\
\hline & $85.33 \%$ & $90.01 \%$ \\
\hline & $86.16 \%$ & $91.18 \%$ \\
\hline & $87.99 \%$ & $93.41 \%$ \\
\hline & $86.55 \%$ & $89.70 \%$ \\
\hline & $86.22 \%$ & $91.11 \%$ \\
\hline & $86.50 \%$ & $90.65 \%$ \\
\hline Average & $86.50 \%$ & $91.27 \%$ \\
\hline
\end{tabular}


The average training and validation accuracy calculated from these sessions have been plotted per epoch in green in Figure 34. The average validation accuracy for the Mel scaled Librosa spectrograms finished at $86.8 \%$ which was very similar to the results in Table 32 . This is important because if the validation accuracy and test accuracy differ by a lot, the model is most likely overfit.

\section{Average Training vs Validation Accuracy for Mel Spectrograms}

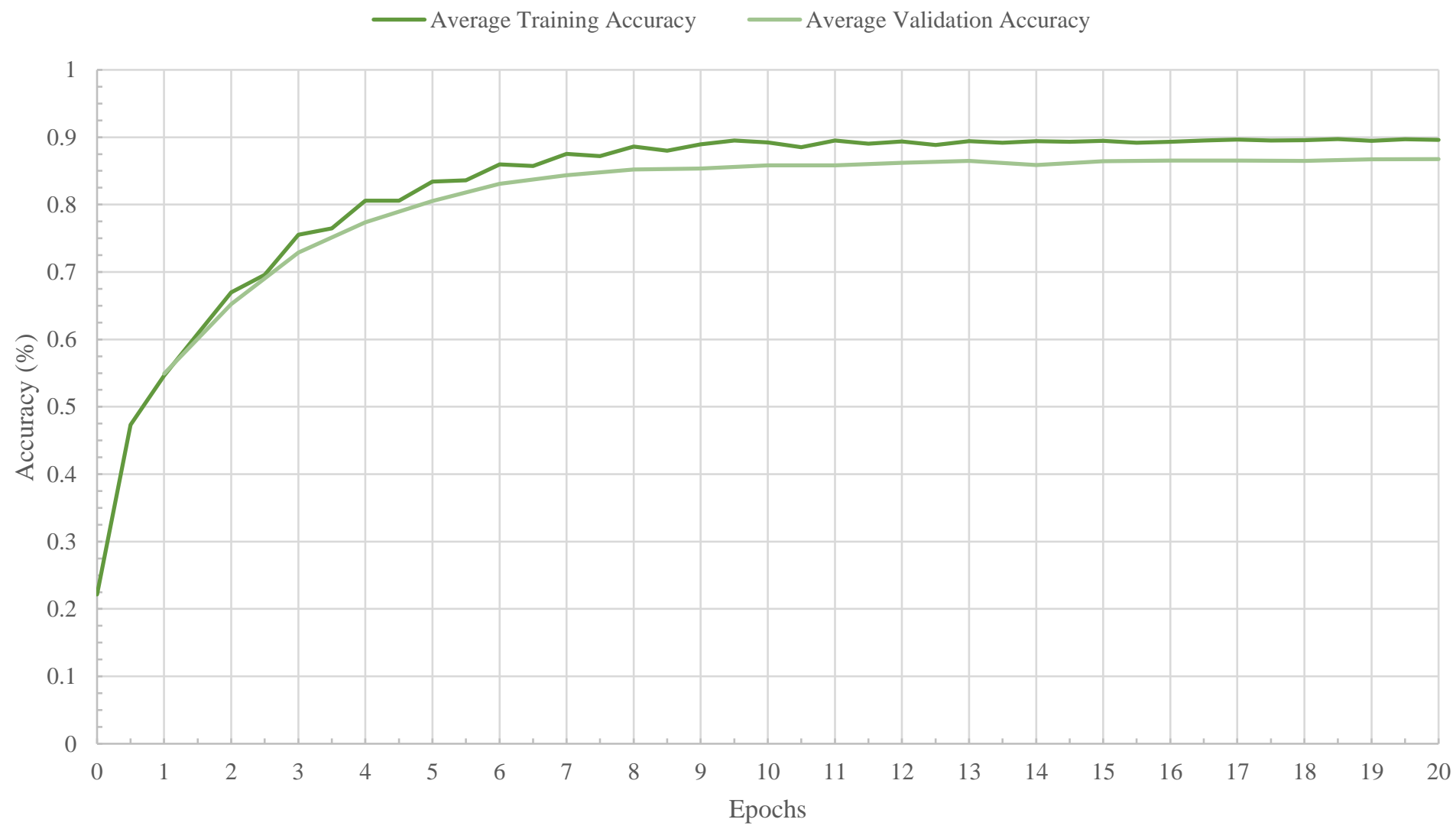

Figure 34 Validation accuracy per epoch for Mel scaled Librosa spectrogram slices.

The average training and validation loss extracted from the ten training sessions presented in Table 32 were plotted in blue in Figure 35. 
Average Training vs Validation Loss for Mel Spectrograms

— Average Training Loss — Average Validation Loss

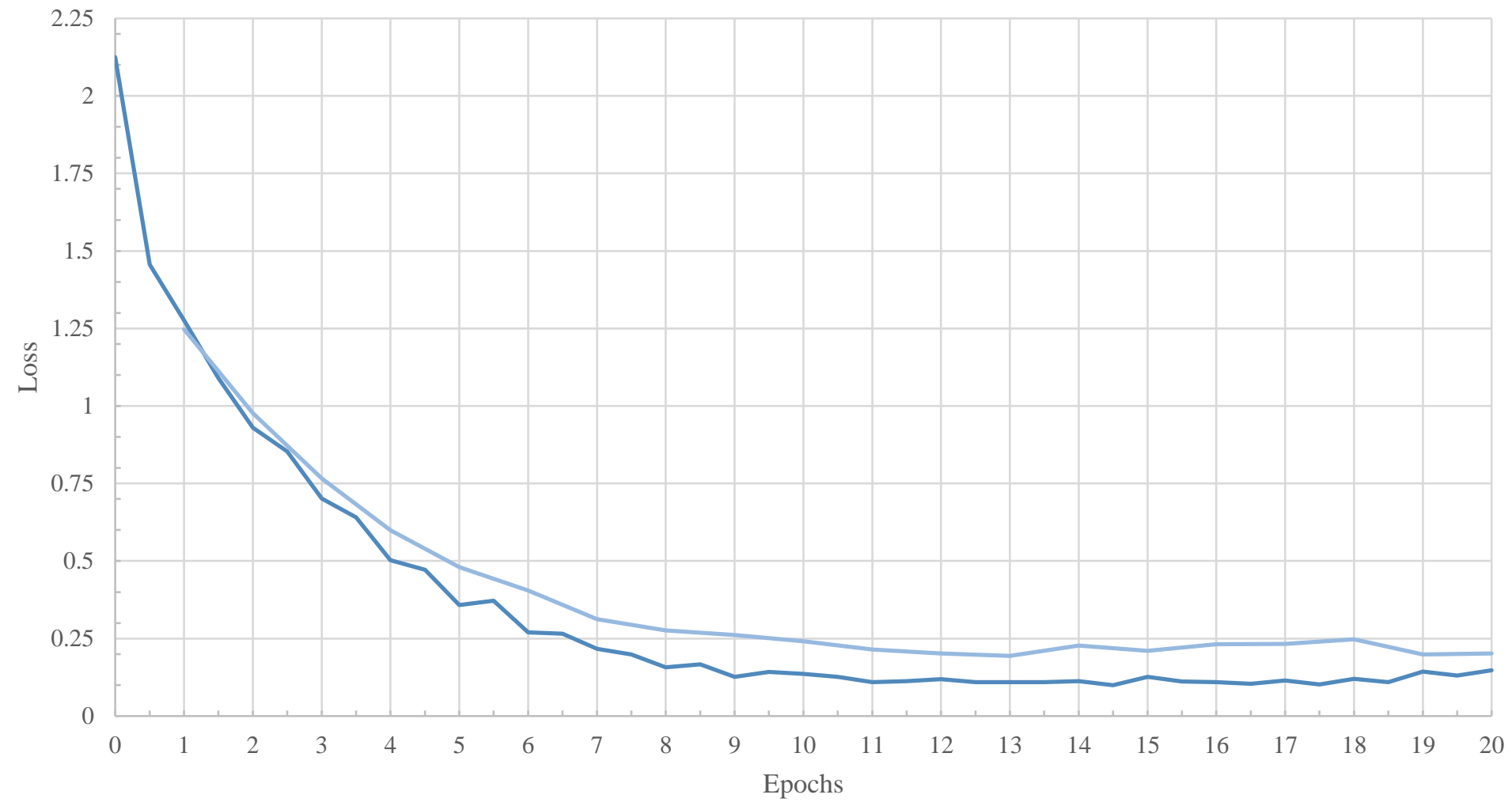

Figure 35 Validation loss per epoch for Mel scaled Librosa spectrogram slices.

\subsubsection{Logarithmic Spectrograms}

Finally, testing the logarithmic Librosa spectrograms to compare to the results already obtained.

The dataset used for logarithmic Librosa spectrograms is shown in Table 33. Each of the datasets

for linear, Mel and logarithmic were all extracted from the same collection so similarly, each of them was lacking slices in the classical genre. 
Table 33 The dataset extracted from the logarithmic Librosa spectrogram slices.

\begin{tabular}{lll} 
Genre & \multicolumn{2}{c}{ Logarithmic Librosa } \\
\hline Genre & Total Slices in Collection B & $\begin{array}{l}\text { Logarithmic Librosa Dataset } \\
* \text { Max } 15,000 \text { per genre }\end{array}$ \\
\hline Alternative & 24,006 & 15,000 \\
Blues & 16,422 & 15,000 \\
Classical & 13,669 & 13,669 \\
Country & 22,104 & 15,000 \\
Electronic & 16,998 & 15,000 \\
Hip Hop & 29,755 & 15,000 \\
Pop & 20,752 & 15,000 \\
Rap & 21,379 & 15,000 \\
RB Soul & 16,966 & 15,000 \\
Rock & 69,662 & 15,000 \\
\hline Total & 251,713 slices & 148,669 slices \\
\hline
\end{tabular}

After training the NN ten times using the dataset above, the results are presented in Table 34 . The average accuracy for logarithmic Librosa spectrograms was $87.40 \%$. The average majority vote accuracy was $91.82 \%$. Notice all ten test accuracies are in the $87 \%$ range which was significantly more consistent than the results from linear or Mel scaled spectrograms. Of the three types of spectrograms, the logarithmic spectrograms produced the most stable accuracy over several training sessions. 
Table 34 Average accuracy and majority vote accuracy obtained used logarithmic Librosa spectrograms.

\begin{tabular}{|ll} 
Test Accuracy & Majority Vote Accuracy \\
\hline $87.75 \%$ & $93.29 \%$ \\
$87.12 \%$ & $91.73 \%$ \\
$87.18 \%$ & $90.22 \%$ \\
$87.34 \%$ & $92.74 \%$ \\
$87.26 \%$ & $91.01 \%$ \\
$87.47 \%$ & $92.24 \%$ \\
$87.59 \%$ & $92.07 \%$ \\
$87.36 \%$ & $91.04 \%$ \\
$87.33 \%$ & $91.25 \%$ \\
$87.62 \%$ & $92.62 \%$ \\
\hline Average & $91.82 \%$ \\
\hline
\end{tabular}

For the training sessions shown in Table 34, the average training accuracy and average validation accuracy obtained after each epoch were plotted in green in Figure 36. The average validation accuracy for logarithmic Librosa spectrograms after 20 epochs was 87.3\%. Also, the average training and validation loss computed from the ten training sessions was graphed in blue in Figure 37. 
Average Training vs Validation Accuracy for Logarithmic Spectrograms

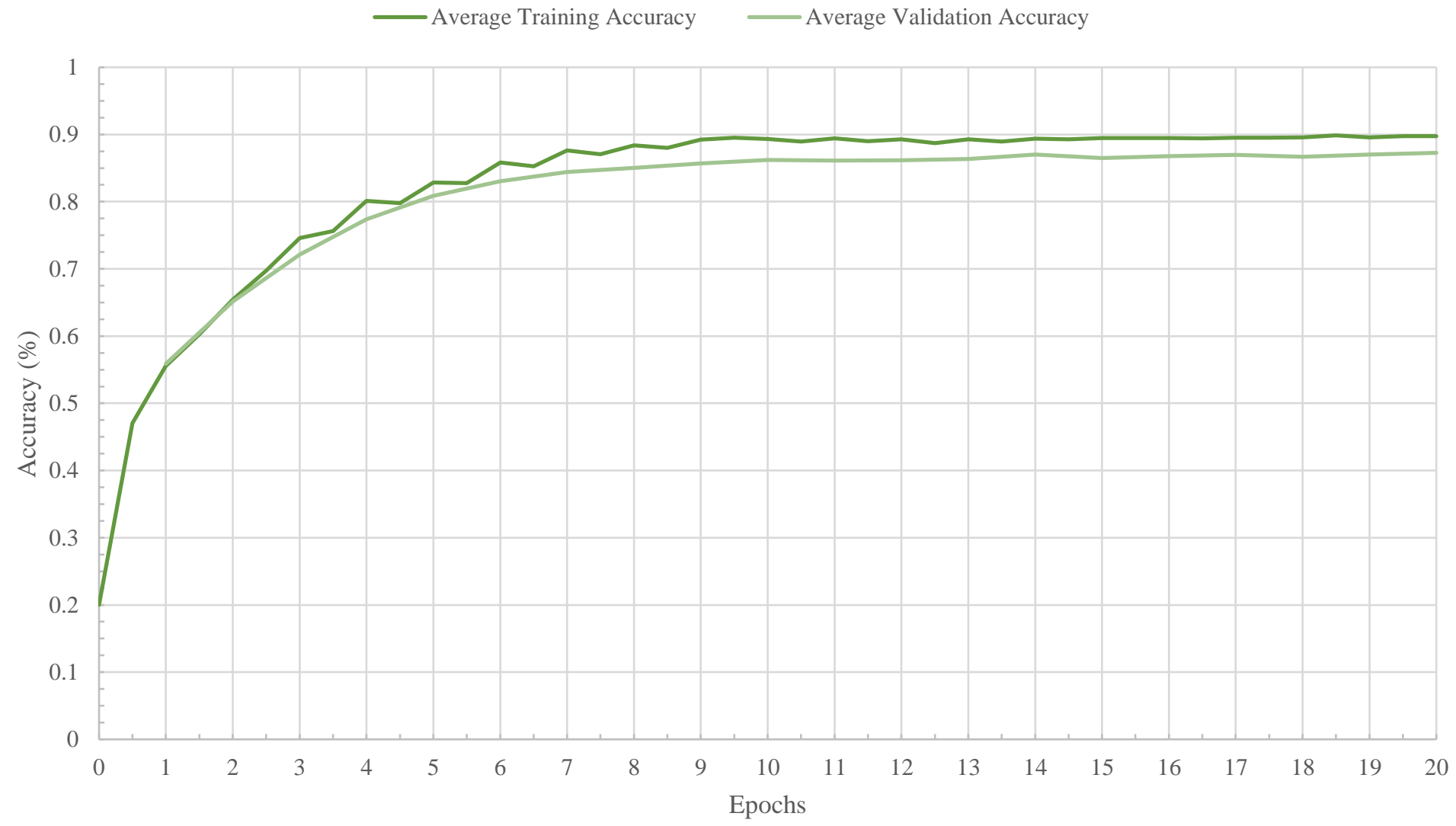

Figure 36 Validation accuracy per epoch for logarithmic Librosa spectrogram slices. 
Average Training vs Validation Loss for Logarithmic Spectrograms

— Average Training Loss _ Average Validation Loss

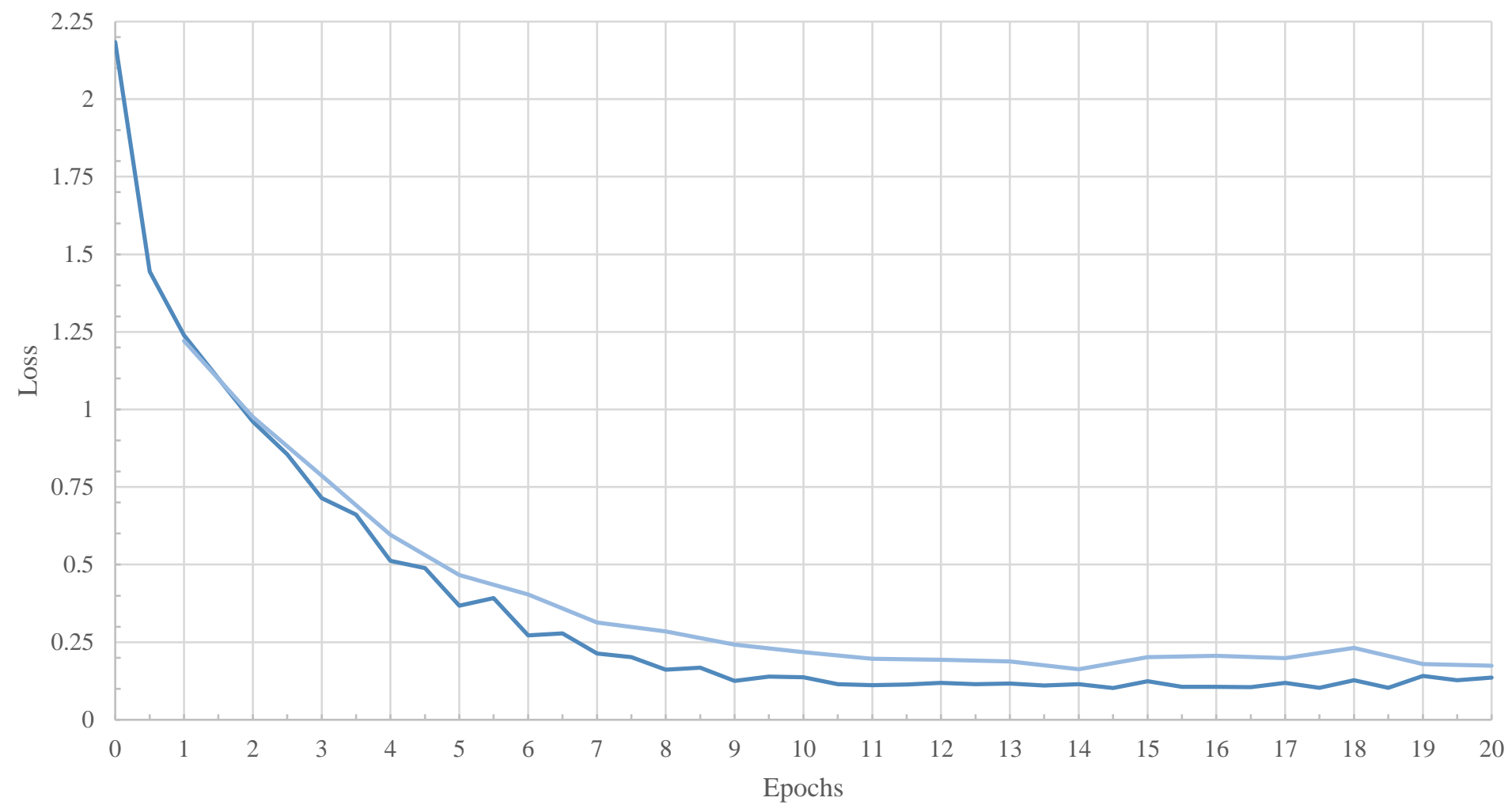

Figure 37 Validation loss per epoch for logarithmic Librosa spectrogram slices.

\subsubsection{Comparison of Linear, Mel, and Logarithmic Spectrograms}

In respect to average accuracy over ten training sessions for each type of spectrogram, logarithmic spectrograms performed the best with an accuracy of $87.40 \%$ shown in Table 35 . Mel was lower at $86.50 \%$ and linear slightly lower than that at $86.13 \%$. For the average majority vote accuracy over ten sessions, the logarithmic spectrograms also produced the most accuracy results shown in Table 35 resulting in $91.82 \%$, followed by Mel at $91.27 \%$, and linear claiming the lowest majority vote accuracy at $90.99 \%$.

The difference between the highest and lowest average accuracy was $1.3 \%$ while the difference between the majority vote accuracy was about $0.8 \%$. A reason for this could be the way majority 
vote accuracy was calculated. Individual incorrectly labelled slices have less affect on the majority vote accuracy than they do for the average accuracy. Imagine one song sliced into 100 snippets in both linear and logarithmic spectrograms. For the linear spectrograms, the trained model could label 20 slices incorrectly. Each of those incorrectly labelled slices will affect the average accuracy in a negative way; however, for the majority vote accuracy the song would be labelled correct because 80 of the 100 song slices were labelled correctly. In this same example for logarithmic, the model could label 10 slices incorrectly which is more accurate than the linear example at 20 incorrectly labelled slices. For the majority vote accuracy, the song would be labelled correctly which is the same for the linear model. This type of example can explain why the majority vote accuracy was more similar for all three types of spectrograms than the average accuracy.

Table 35 A comparison of accuracy and majority vote accuracy across linear, Mel scaled, and logarithmic Librosa spectrograms.

\begin{tabular}{lcc} 
& Average Accuracy & Majority Vote Accuracy \\
\hline Linear & $86.13 \%$ & $90.99 \%$ \\
Mel Scaled & $86.50 \%$ & $91.27 \%$ \\
Logarithmic & $\mathbf{8 7 . 4 0 \%}$ & $\mathbf{9 1 . 8 2 \%}$ \\
\hline \multicolumn{2}{c}{ For the ten training sessions for each of the spectrogram types, the difference between the highest } \\
and lowest accuracy is presented in Table 36. Overall, Mel spectrograms seems to be the most \\
inconsistent while training. The Mel spectrograms differed by 2.66\% and linear differed by a total \\
of 2.06\%. The logarithmic spectrograms are the most consistent with a difference of $0.63 \%$ between \\
training sessions.
\end{tabular}

Table 36 Difference between training session accuracies for each spectrogram type.

Total Difference in Training Session Accuracies

\begin{tabular}{ll}
\hline Linear & $2.06 \%$ \\
Mel Scaled & $2.66 \%$ \\
Logarithmic & $\mathbf{0 . 6 3 \%}$ \\
\hline
\end{tabular}


The average training and validation accuracy graphs presented in sections 4.8 .1 to 4.8 .3 were compiled into one graph in Figure 38 for comparison. The graph is focused on accuracies between 0.6 and 1.0 to clearly view the differences. The final average validation accuracy for logarithmic is noticeably higher than both the Mel and linear. Mel has the next highest validation accuracy with linear falling slightly lower.

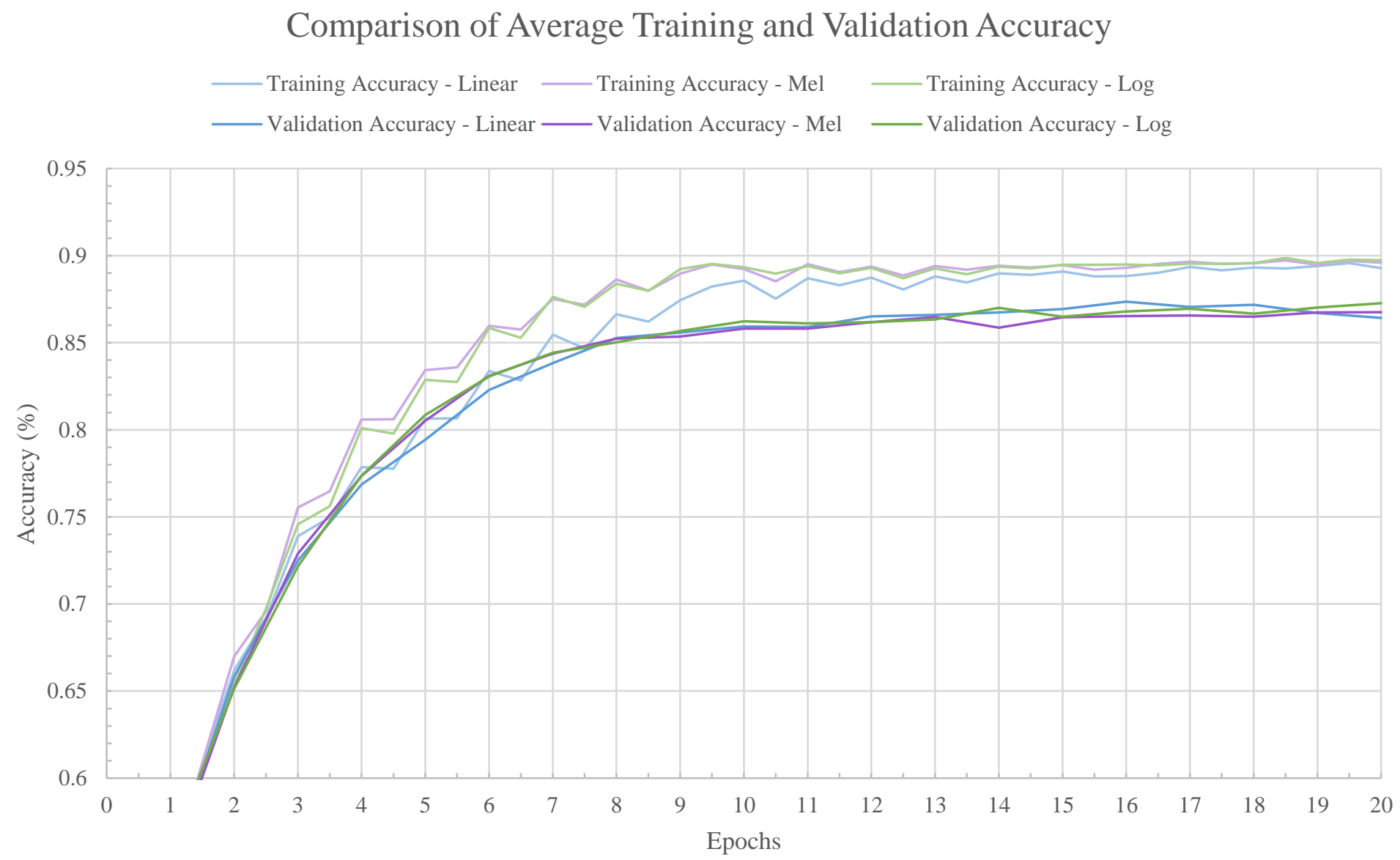

Figure 38 Comparison of validation accuracy per epoch for linear, Mel, and logarithmic Librosa spectrogram slices.

Similar to the graph above, each of the average training and validation loss graphs from sections 4.8.1 to 4.8.3 were accumulated in Figure 39. The lower the loss, the better the final NN model is at labelling unseen data. The graph clearly shows the logarithmic spectrograms achieve the best 
loss by epoch 20 . This was followed by a slightly higher loss reached by Mel, and finally linear with the highest validation loss value.

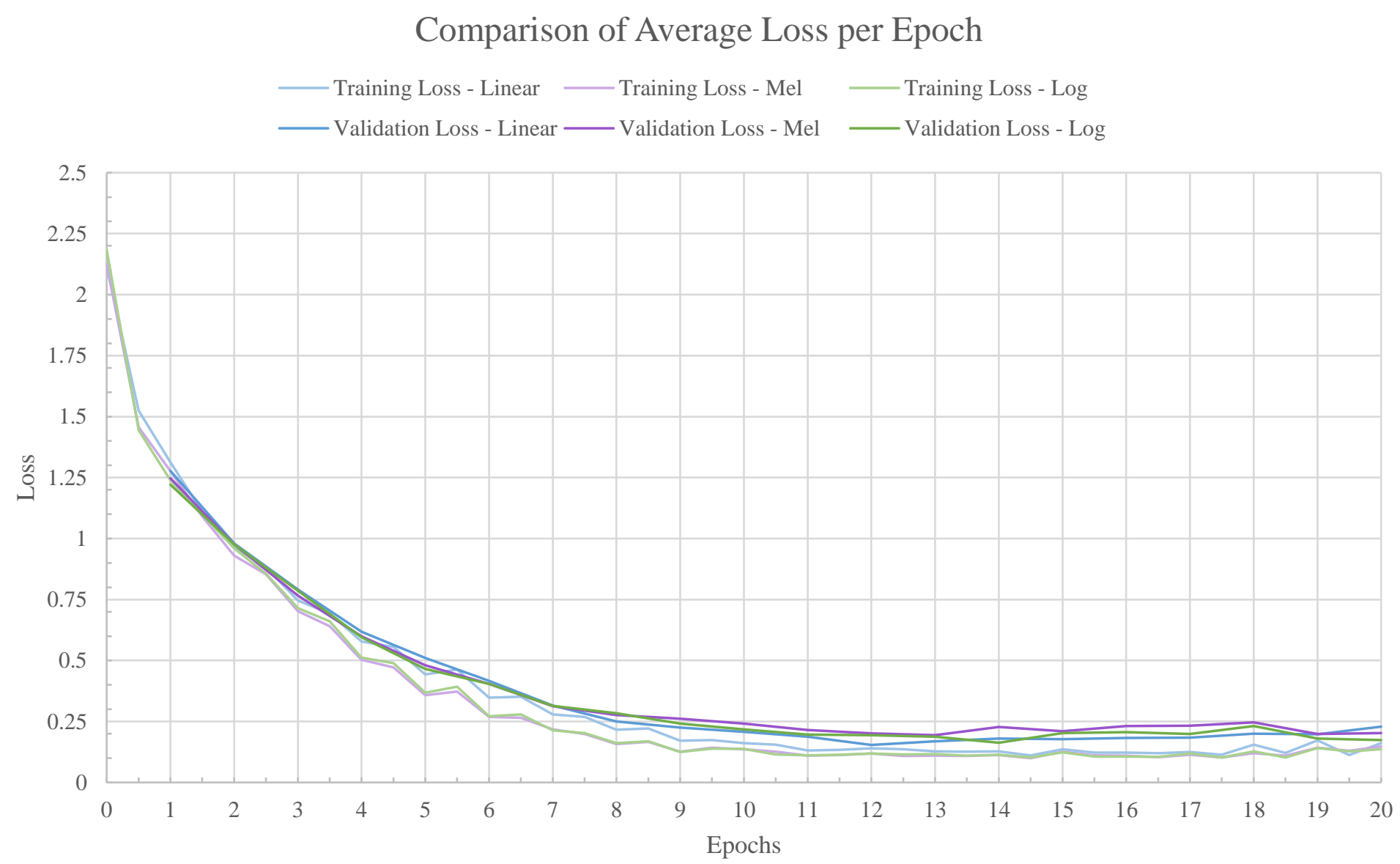

Figure 39 Comparison of validation loss per epoch for linear, Mel, and logarithmic Librosa spectrogram slices.

\subsection{Confusion Matrix}

Another assessment tool used on trained NN models are confusion matrices. Confusion matrices are extracted from classification networks to help understand exactly where the NN is predicting the labels incorrectly. The confusion matrix is a grid of values representing the number of predicted labels in respect to the actual labels of the output classes. The genre classification problem presented in this thesis has 10 genre output classes so the grid was formatted in a $10 \times 10$ matrix of values. The column headers represent the predicted labels and the row headers represent the actual 
labels. Once the NN has been trained, TensorFlow can assess the model on the test dataset and produce a table filled with the predicted versus actual labels.

\subsubsection{Linear Spectrograms}

Once the NN was trained on linear spectrograms, the average confusion matrix for each of the ten training sessions was put into one matrix in Figure 40. Three output classes have been shortened for the display purposes:

- Altern. for Alternative

- Classic. for Classical

- Electro. for Electronic

\begin{tabular}{|c|c|c|c|c|c|c|c|c|c|c|}
\hline \multicolumn{11}{|c|}{ Predicted Labels } \\
\hline & Altern. & Blues & Classic. & Country & Electro. & НiрНор & Pop & Rap & RB Soul & Rock \\
\hline Altern. & 1,261 & 22 & 14 & 23 & 8 & 25 & 35 & 3 & 6 & 104 \\
\hline Blues & 17 & 1,373 & 5 & 10 & 5 & 17 & 16 & 4 & 6 & 42 \\
\hline Classic. & 7 & 3 & 1,327 & 5 & 4 & 2 & 7 & 1 & 0 & 5 \\
\hline Country & 18 & 25 & 9 & 1,323 & 14 & 13 & 33 & 17 & 13 & 37 \\
\hline Electro. & 13 & 7 & 13 & 35 & 1,301 & 26 & 21 & 20 & 26 & 38 \\
\hline НірНор & 17 & 22 & 4 & 18 & 18 & 1,267 & 29 & 21 & 73 & 49 \\
\hline Pop & 46 & 21 & 12 & 45 & 31 & 31 & 1,185 & 22 & 33 & 77 \\
\hline Rap & 8 & 7 & 2 & 31 & 20 & 49 & 31 & 1,268 & 61 & 27 \\
\hline RB Soul & 7 & 9 & 4 & 20 & 17 & 67 & 25 & 36 & 1,296 & 19 \\
\hline Rock & 105 & 35 & 26 & 33 & 25 & 29 & 64 & 6 & 15 & 1,163 \\
\hline
\end{tabular}

The darker blue squares running diagonal from top left to bottom right are the values of correct predictions. For example, the first value which was 1,261 means the NN predicted Alternative 1,261 when the actual label was Alternative. All values outside of this diagonal were the number of incorrectly labelled slices. Values that were outside of this diagonal and have a higher value than 
65 have been highlighted in light blue to visually separate them. There were five lighter blue squares in this confusion matrix with the first one being in the top right with a value of 104 . This means the NN predicted Rock for 104 slices when the actual label was Alternative. For this confusion matrix the genres which were commonly mislabelled are:

- 105 slices predicted as Alternative but the actual label was Rock

- 104 slices predicted as Rock but the actual label was Alternative

- 77 slices predicted as Rock but the actual label was Pop

- 73 slices predicted as RB Soul but the actual label was Hip Hop

- 67 slices predicted as Hip Hop but the actual label was RB Soul

\subsubsection{Mel Spectrograms}

The average confusion matrix extracted from the ten training sessions using Mel scaled spectrograms is shown in Figure 41.

\begin{tabular}{c|c|c|c|c|c|c|c|c|c|c|} 
& Altern. & Blues & Classic. & Country & Electro. & HipHop & Pop & Rap & RB Soul & Rock \\
\hline Altern. & 1,260 & 22 & 7 & 21 & 16 & 18 & 34 & 8 & 7 & 104 \\
\hline Blues & 19 & 1,384 & 4 & 18 & 4 & 13 & 14 & 5 & 3 & 36 \\
\hline Classic. & 8 & 5 & 1,307 & 5 & 7 & 3 & 6 & 1 & 1 & 8 \\
\hline Country & 19 & 20 & 4 & 1,348 & 18 & 4 & 31 & 13 & 5 & 39 \\
\hline \multirow{\Xi}{\Xi}{ Electro. } & 10 & 6 & 6 & 24 & 1,336 & 20 & 28 & 29 & 20 & 23 \\
\hline HipHop & 12 & 18 & 3 & 14 & 23 & 1,222 & 37 & 62 & 76 & 34 \\
\hline Pop & 44 & 18 & 8 & 48 & 31 & 32 & 1,180 & 34 & 22 & 85 \\
\hline Rap & 4 & 3 & 2 & 14 & 15 & 28 & 42 & 1,336 & 45 & 12 \\
\hline RB Soul & 6 & 5 & 2 & 11 & 15 & 66 & 37 & 58 & 1,286 & 15 \\
\hline Rock & 107 & 29 & 22 & 50 & 29 & 30 & 67 & 16 & 6 & 1,141 \\
\hline
\end{tabular}

Figure 41 The average confusion matrix after training on Mel Librosa spectrograms. 
Similar to the previous matrix, the values that are outside of this diagonal and have a value of 65 and higher have been highlighted in light blue. There were six light blue squares in this confusion matrix. For this confusion matrix the genres which were commonly mislabelled are:

- 107 slices predicted as Alternative but the actual label was Rock

- 104 slices predicted as Rock but the actual label was Alternative

- 85 slices predicted as Rock but the actual label was Pop

- 76 slices predicted as RB Soul but the actual label was Hip Hop

- 67 slices predicted as Pop but the actual label was Rock

- 66 slices predicted as Hip Hop but the actual label was RB Soul

\subsubsection{Logarithmic Spectrograms}

Finally, the confusion matrix which has been averaged over the ten training sessions for the logarithmic spectrograms is presented in Figure 42.

\begin{tabular}{|c|c|c|c|c|c|c|c|c|c|c|}
\hline & & & & $\operatorname{Pre}$ & dicted $L c$ & & & & & \\
\hline & Altern. & Blues & Classic. & Country & Electro. & НipHор & Pop & Rap & RB Soul & Rock \\
\hline Altern. & 1,250 & 16 & 6 & 23 & 16 & 20 & 38 & 10 & 7 & 116 \\
\hline Blues & 13 & 1,418 & 2 & 11 & 2 & 5 & 9 & 4 & 3 & 32 \\
\hline Classic. & 3 & 4 & 1,345 & 2 & 3 & 2 & 4 & 1 & 0 & 3 \\
\hline Country & 21 & 16 & 5 & 1,336 & 13 & 6 & 30 & 11 & 6 & 56 \\
\hline Electro. & 9 & 4 & 6 & 24 & 1,347 & 22 & 25 & 18 & 17 & 27 \\
\hline НipHop & 8 & 15 & 5 & 12 & 24 & 1,289 & 22 & 42 & 51 & 33 \\
\hline Pop & 53 & 26 & 9 & 55 & 25 & 31 & 1,168 & 32 & 26 & 77 \\
\hline Rap & 4 & 6 & 2 & 13 & 16 & 36 & 28 & 1,340 & 42 & 14 \\
\hline RB Soul & 8 & 3 & 1 & 9 & 19 & 43 & 27 & 45 & 1,332 & 15 \\
\hline Rock & 105 & 28 & 23 & 57 & 24 & 23 & 59 & 11 & 9 & 1,162 \\
\hline
\end{tabular}

Figure 42 The average confusion matrix after training on logarithmic Librosa spectrograms. 
There were three light blue squares which have been highlighted in this confusion matrix because they have values higher than 65 . This is to visually separate them and draw attention to the genres which were highly mislabelled. For this confusion matrix the common mislabelled genres:

- 116 slices predicted as Rock but the actual label was Alternative

- 105 slices predicted as Alternative but the actual label was Rock

- 77 slices predicted as Rock but the actual label was Pop

\subsubsection{Comparison of Confusion Matrices}

A common trend across all spectrograms was the network labelling Rock songs as Alternative and the inverse. This could be attributed to the actual labels assigned to the raw song data. Depending on how the songs were labelled or who labelled them, the raw songs could be labelled incorrectly or even border the line of what defines Rock and Alternative as a genre. Other genres which were being commonly interchanged by the network were Pop and Rock, followed by Hip Hop with RB Soul. In general, the genres which were being incorrectly labelled were being mistaken for other similar genres. For example, Hip Hop and RB Soul are similar genres which were confusing the matrix occasionally; however, it was not common for the network to mislabel a Classical song as Rock since those genres fall into fairly opposing categories of music.

The following results in Table 37 show the total incorrectly labelled slices per genre for the test dataset. These values were calculated by summing each matrix row and subtracting the diagonal of dark blue values which represent correctly labelled data points. The total at the bottom represents the total number of slices which were incorrectly labelled within the test dataset. For linear spectrograms, there was a total of 2,116 slices incorrectly labelled. Following this was Mel with 2,041 incorrect and then logarithmic with 1,880. As expected, these will follow the same trend as the average accuracy since the confusion matrices were also created from the test dataset. 
Table 37 The total slices by genre which are incorrectly labelled for the three types of spectrograms.

\begin{tabular}{llll} 
Genre & Linear Slices & Mel Slices & Logarithmic Slices \\
\hline Alternative & 239 & 235 & 251 \\
Blues & 121 & 116 & 82 \\
Classical & 34 & 42 & 21 \\
Country & 177 & 152 & 164 \\
Electronic & 199 & 165 & 153 \\
Hip Hop & 251 & 278 & 211 \\
Pop & 317 & 320 & 332 \\
Rap & 235 & 164 & 160 \\
RB Soul & 204 & 214 & 169 \\
Rock & 337 & 355 & 338 \\
Total & $\mathbf{2 , 1 1 6}$ slices & $\mathbf{2 , 0 4 1}$ slices & $\mathbf{1 , 8 8 0}$ slices \\
\hline \multicolumn{2}{c}{ A common trend across all the spectrograms is that Rock was the most incorrectly labelled genre } \\
and the most correctly labelled genre was Classical. Classical music is arguably the most unique \\
genre within this dataset so it makes sense that it was labelled correctly the majority of the time.
\end{tabular}




\section{CONCLUSIONS AND FUTURE WORK}

\subsection{Conclusion}

This thesis described the research and implementation work done in machine learning with respect to music data and genre classification. This research involved taking music previously labelled by genre, converting them into spectrograms, and slicing the spectrograms into shorter time segments which represent 2.56 seconds of raw audio data. The genre label is then attached to each of these spectrograms which are used as inputs into the proposed convolutional neural network.

The convolutional neural network proposed in this thesis can identify a total of 10 genres which include Alternative, Classical, Country, Dance, Electronic, Hip Hop, Pop, Rap, RB Soul, and Rock. Using only 2.56 seconds of a song, the NN is able to classify new musical data into one of the 10 genres with an accuracy of $87.4 \%$. If the $\mathrm{NN}$ is presented with a full song instead of only one short time segment of the song, the accuracy increases to $91.8 \%$. This proposed CNN can be used to label new music which has never been labelled before.

The proposed network is comprised of six convolutional layers followed by a fully connected layer and then finally a softmax function to calculate the probability of each genre detected. The network outputs a one-hot array which represents the predicted genre label of the spectrogram slice. There was exploration done into the number of convolutional layers within the network which would produce the highest accuracy. The results from those experiments showed six layers were optimal for the proposed network presented in this thesis.

During this research the tuning of the proposed network included a comparison of different hyperparameters, length of spectrogram slices being analyzed, and the windowing function used to create the spectrogram were all compared, measured, and determined. An overview of the 
hyperparameters selected is shown in Table 14. The length of spectrogram input used was a $128 \mathrm{x}$ 128 pixel image which represented 2.56 seconds of raw audio and finally, the windowing function utilized was the Hanning window function.

Two separate datasets were tested with the proposed network to prove the same network architecture could be applied to different music libraries successfully. The music libraries used were gathered separately from different sources. This is important to note because depending on where the music was sourced from, it can affect how the music was initially labelled; whether that be by a professional company or by an inexperienced person. Other changes to the dataset included comparing an evenly distributed dataset with an uneven dataset. It was determined when the songs within a training dataset are unevenly distributed, the accuracy of the network is negatively affected.

Experiments presented in this thesis have also demonstrated the difference in performance of different spectrogram representations of audio data using the proposed convolutional neural network. Linear, Mel scaled, and logarithmic spectrograms have been discussed, analyzed and compared with results that demonstrate that logarithmic spectrograms produce better and more consistent test accuracies.

The results obtained using logarithmic spectrograms were $87.4 \%$ accurate on the test data with a majority vote accuracy of $91.8 \%$. This accuracy was followed by Mel with a test accuracy of $86.5 \%$ and a majority vote accuracy of $91.3 \%$. Finally, linear spectrograms are the least accurate with $86.1 \%$ test accuracy and $91.0 \%$ majority vote accuracy. The logarithmic spectrograms produced the leading results indicating that it is beneficial to learn from spectrograms that emphasize the lower frequencies in music rather than having all frequencies equally represented.

Finally, this research summarizes which genres the network was mislabelling. It shows there are a few genres which get swapped while the network is labelling spectrogram slices. Rock and 
Alternative are two of the most common genres that appear to get mislabelled. Another common pair of genres which are mislabelled are Hip Hop and RB Soul. In each of these examples, the confusion matrix shows the network has a hard time separating and classifying these genres correctly. Adding more songs into those genres within the dataset might help the NN distinguish the key differences between the genres which could improve accuracy. With the example of Rock and Alternative, a potential solution could be combining the two into one main genre labelled Rock. This solution would be appropriate since Alternative is arguably a sub-genre of Rock; however, it would not be appropriate for Hip Hop and RB Soul for example. Another contributing issue could potentially be with the actual labels on the music itself. Depending on who or what labelled the music, a song which is labelled Rock could actually be classified as Alternative since genre definitions are ever changing and subjective.

\subsection{Future Work}

Future research work with respect to modifications to the algorithms and feature engineering include experimenting with different filters while converting the MP3 file to a spectrogram. For example, applying a high pass or low pass filter to better understand the effects of these modifications. The proposed network has potential to classify songs by genre with a higher accuracy using only the high frequencies or low frequencies of a dataset.

With respect to the dataset, future work could include changes to the genres being used. From the analysis of the confusion matrix, Rock and Alternative were discovered to be mislabelled frequently. There are a few options that could help with this as mentioned in 5.1. For Rock and Alternative, it would be interesting to view the effects if those two genres were combined into one (Rock) when training the network. Additionally, there are songs within the current collection which have a genre label such as 'Rap/Hip Hop'. The songs with these type of genre labels had to be 
categorized into one single genre for training. For the 'Rap/Hip Hop' example, all the songs were classified into Rap. If the music library was large enough in the future, these types of songs which border the main genres could be ignored to weigh the outcome they have on accuracy.

Future work could include implementing different music collections such as the GTZAN dataset. The GTZAN was not expressly created for music genre recognition but due to its widespread availability the dataset has become somewhat of a benchmark when classifying music by genre. There are several works that compare their results using the GTZAN dataset; however, it has been proven that the dataset is not as flawless as originally thought in 2002 when it was created. "Few researchers have ever listened to or critically evaluated the contents of GTZAN, and thus its faults remained undiscovered" until 2013 [55]. After a thorough analysis of the GTZAN dataset by Sturm [55], flaws like repetition, potential mislabellings, and distortions were identified within GTZAN. However, numerous papers still used the GTZAN dataset after this analysis was released. Instead of suggesting banishing the dataset, Sturm recommended taking into consideration the contents of the GTZAN dataset when using it for research purposes.

In addition, future work may include replacing the current $\mathrm{CNN}$ architecture with a recurrent neural network. A RNN is commonly used for tasks where sequential context is important. Using an RNN means while the network is learning it is being influenced by previous training iterations. RNNs are commonly applied to text and speech data since the order of words in sentences are important for conveying meaning. RNNs can be applied to music tasks since the order of notes played within a song create the rhythm of the piece which is important and unique to each genre and song. A genre classification RNN may use Musical Instrument Digital Interface (MIDI) files as input. A MIDI file contains song data formatted in a way that each note can be extracted from a given song file. The notes can be fed into an RNN which can learn from the data in a sequential 
order. The accuracy from the RNN could be compared with the currently proposed CNN's accuracy on the same dataset. This is an area of research already being investigated [56, 57].

In a similar area of research which involves music classification using NNs, a binary classification $\mathrm{NN}$ could be tasked with determining if a user likes a song based on previous preferences. This is an interesting area of research to explore; however, the dataset collected within this thesis would not suffice. The dataset required for this task would need to contain songs in which the user has listened to and subsequently marked as either liking or disliking. It would potentially be significantly time consuming to gather this type of a dataset as a user would need to listen to approximately 2,000 songs which could be about 115 hours of song data (with an average of 3.45 minutes per song). While gathering the dataset the user would need to deliberately listen to every song, even to mark it as dislike. A similar type of CNN to the one proposed by this thesis could be used; however, the number of output classes would change from 10 to 2 which would represent whether the user likes the given song. 


\section{REFERENCES}

[1] M. Lopes, F. Gouyon, A. L. Koerich et al., Selection of Training Instances for Music Genre Classification, p.^pp. 4569-4572, 2010.

[2] G. Tzanetakis, G. Essl, and P. Cook, "Automatic Musical Genre Classification Of Audio Signals," in 2nd International Society for Music Information Retrieval Conference, Indiana University, Bloomington, Indiana, 2002.

[3] W. Wu, F. Han, G. Song et al., "Music Genre Classification Using Independent Recurrent Neural Network," in 2018 Chinese Automation Congress (CAC), Xi'an, China, China, 2018, pp. 192-195.

[4] Y. M. G. Costa, L. S. Oliveria, A. L. Koerich et al., "Comparing Textural Features for Music Genre Classification," in The 2012 International Joint Conference on Neural Networks (IJCNN), Brisbane, Australia, 2012.

[5] W.-H. D. Hearing. "The human hearing range - what can you hear?," December 13, 2020; https://global.widex.com/en/blog/human-hearing-range-what-can-you-hear.

[6] S. Gollapudi, "Practial Machine Learning," Packt Publishing Ltd., 2016.

[7] SAS. "SAS | The Power To Know," Sept 19, 2019; https://www.sas.com/en_ca/insights/analytics/machine-learning.html.

[8] S. Shukla, "What is Machine Learning and Why it matters," September 16, upGrad Blog, 2018.

[9] S. Mingguang, and Z. Bing, "Semi-supervised learning improves gene expression-based prediction of cancer recurrence," Bioinformatics, vol. 27, no. 21, pp. 6, 2011.

[10] Y. LeCun, Y. Bengio, and G. Hinton, "Deep learning," Nature, vol. 521, no. 7553, pp. 436, May 2015, 2015.

[11] I. Goodfellow, Y. Bengio, and A. Courville, Deep Learning: The MIT Press, 2016.

[12] S. Sharma, "Epoch vs Batch Size vs Iterations," https://towardsdatascience.com/epochvs-iterations-vs-batch-size-4dfb9c7ce9c9, [July 4, 2020, 2017].

[13] J. Brownlee, "How to Configure the Learning Rate When Training Deep Learning Neural Networks," July 4, Machine Learning Mastery, 2019.

[14] Katanforoosh, and Kunin, "Initializing neural networks," https:/www.deeplearning.ai/ainotes/initialization/, [July 4, 2020, 2019].

[15] J. Brownlee, "A Gentle Introduction to Dropout for Regularizing Deep Neural Networks," July 4, Machine Learning Mastery, 2018. 
[16] J. Brownlee, "Loss and Loss Functions for Training Deep Learning Neural Networks," October 4, Machine Learning Mastery, 2019.

[17] M. A. Nielsen, "Neural Networks and Deep Learning," D. Press, ed., Determination Press, 2015.

[18] J. Dai, W. Liu, C. Ni et al., “"Multilingual" Deep Neural Network for Music Genre Classification," in Interspeech 2015, Dresden, Germany, 2015, pp. 2907-2911.

[19] S. Vishnupriya, and K. Meenakshi, "Automatic Music Genre Classification using Convolution Neural Network." p. 4, 2018.

[20] Y. M. G. Costa, L. S. Oliveria, and C. N. Silla Jr., "An Evaluation of Convolutional Neural Networks for Music Classification Using Spectrograms," 52, https://www.sciencedirect.com/science/article/pii/S1568494616306421, [December 16, 2018, 2017].

[21] R. L. Aguiar, Y. M. G. Costa, and C. N. Silla Jr., "Exploring Data Augmentation to Improve Music Genre Classification with ConvNets," in International Joint Conference on Neural Networks (IJCNN), Rio de Janeiro, Brazil, 2018.

[22] J. Brownlee, "What is a Confusion Matrix in Machine Learning," June 7, M. L. Mastery, ed., Machine Learning Mastery, 2016.

[23] J. Shah, "Neural Networks for Beginners: Popular Types and Applications," https://blog.statsbot.co/neural-networks-for-beginners-d99f2235efca, [January 12, 2019, 2017].

[24] O. Mogren, "C-RNN-GAN: Continuous recurrent neural networks with adversarial training," CoRR, vol. abs/1611.09904, 2016.

[25] Y. Chen, “Towards Explaining Neural Networks," Faculty of Science, Utrecht University, Utrecht, Netherlands, 2017.

[26] T. Mikolov, M. Karafiat, L. Burget et al., "Recurrent neural network based language model," INTERSPEECH, vol. 2, pp. 4, 2010.

[27] A. Tchircoff, "The mostly complete chart of Neural Networks, explained," https://towardsdatascience.com/the-mostly-complete-chart-of-neural-networks-explained3fb6f2367464, [January 8, 2019, 2017].

[28] M. Müller, Fundamentals of Music Processing: Audio, Analysis, Algorithms, Applications: Springer International Publishing, 2015.

[29] Librosa. "Librosa," May 17, 2020; https://librosa.github.io/librosa/.

[30] I. Dees, "Window Functions in Spectrum Analyzers," December 8, Textronix, 2013. 
[31] L. Norskog. "SoX - Sound eXchange," https://linux.die.net/man/1/rec.

[32] Audacity. "Spectrogram View," May 17, 2020; https://alphamanual.audacityteam.org/man/Spectrogram_View.

[33] P. Nair, "The dummy's guide to MFCC," February 9, 2020, Medium, ed., Medium, 2018.

[34] D. Gartzman, "Getting to Know the Mel Spectrogram," https://towardsdatascience.com/getting-to-know-the-mel-spectrogram-31bca3e2d9d0, [February 9, 2020, 2019].

[35] D. O'Shaughnessy, Speech Communications: Human and Machine, p.^pp. 548: WileyIEEE Press, 2000.

[36] A. Elbir, B. Çam Hilmi, M. Emre İyican et al., "Music Genre Classification and Recommendation by Using Machine Learning Techniques," in 2018 Innovations in Intelligent Systems and Applications Conference (ASYU), 2018, pp. 1-5.

[37] J. Anden, and S. Mallat, "Deep Scattering Spectrum," IEEE Transactions on Signal Processing, vol. 62, no. 16, 2014.

[38] Y. M. G. Costa, L. S. Oliveria, A. L. Koerich et al., "Music genre recognition using spectrograms," 2011 18th International Conference on Systems, Signals and Image Processing, pp. 1-4, 2011, 2011.

[39] T. O'Brien, "Learning to understand music from Shazam," https://blog.shazam.com/learning-to-understand-music-from-shazam-56a60788b62f, [December 19, 2018, 2017].

[40] G. Song, Z. Wang, F. Han et al., "Music auto-tagging using deep Recurrent Neural Networks," Neurocomputing, vol. 292, pp. 6, 2018, 2018.

[41] S. Sigtia, and S. Dixon, "Improved Music Feature Learning With Deep Neural Networks," in IEEE International Conference on Acoustic, Speech and Signal Processing, Florence, Italy, 2014, pp. 6959-6963.

[42] J. Despois. "Finding the genre of a song with Deep Learning - A.I. Odyssey part. 1," December 27, 2018; https://hackernoon.com/finding-the-genre-of-a-song-with-deeplearning-da8f59a61194.

[43] S. Panwar, A. Das, M. Roopaei et al., "A deep learning approach for mapping music genres," in 12th System of Systems Engineering Conference, Waikoloa, HI, USA, 2017.

[44] K. Choi, G. Fazekas, and M. Sandler, "Automatic tagging using deep convolutional neural networks," CoRR, vol. abs/1606.00298, 2016. 
[45] S. Dieleman, and B. Schrauwen, "End-to-end learning for music audio," in IEEE International Conference on Acoustics, Speech and Signal Processing (ICASSP), Florence, Italy, 2014.

[46] M. H. Nguyen, and F. de la Torre, "Optimal Feature Selection for Support Vector Machines," Pattern Recognition, vol. 43, no. 3, pp. 8, March, 2010, 2010.

[47] Y. Lavner, and D. Ruinskiy, "A Decision-tree-based Algorithm for Speech/Music Classification and Segmentation," EURASIP J. Audio Speech Music Process., vol. 2009, no. January, pp. 14, January 2009, 2009.

[48] T. Li, M. Ogihara, and Q. Li, "A Comparative Study on Content-Based Music Genre Classification," in International ACM SIGIR Congerence on Research and Development in Information Retrieval Toronto, Canada, 2003, pp. 282-289.

[49] S. Masood, A. Saleem, M. Sheezan et al., "Genre classification of songs using neural network," in International Conference on Computer and Communication Technology, Allahabad, 2014, pp. 285-289.

[50] T. Raissi, A. Tibo, and P. Bientinesi, "Extended Pipeline for Content-Based Feature Engineering in Music Gene Recognition," in International Conference on Acoustics, Speech and Signal Processing, Calgary, Canada, 2018, pp. 2661-2665.

[51] MARSYAS, "GTZAN," MARSYAS, ed., 2002.

[52] ISMIR, "ISMIR 2004 Genre Dataset," ISMIR, ed., 2004.

[53] G. Tzanetakis, and P. Cook, "Musical Genre Classification of Audio Signals," IEEE Transactions on Speech and Audio Processing, vol. 10, no. 5, pp. 10, 2002, 2002.

[54] TFlearn. "TFlearn," June 6, 2020; https://github.com/tflearn/tflearn.

[55] B. L. Sturm, "The GTZAN dataset: Its contents, its faults, their effects on evaluation, and its future use," CoRR, vol. abs/1306.1461, 2013.

[56] M. K. Jędrzejewska, A. Zjawiński, and S. Bartłomiej, "Generating Musical Expression of MIDI Music with LSTM Neural Network," in International Conference on Human System Interaction, Gdansk, Poland, 2018.

[57] W. Zhao, Y. Zhou, Y. Tie et al., "Recurrent Neural Network for MIDI Music Emotion Classification," in 2018 IEEE 3rd Advanced Information Technology, Electronic and Automation Control Conference (IAEAC), Chongqing, China, 2018. 